\title{
Gromov, Cauchy and causal boundaries for Riemannian, Finslerian and Lorentzian manifolds
}

\author{
J.L. Flores ${ }^{*}$, J. Herrera* ${ }^{*}$ M. Sánchez ${ }^{\dagger}$ \\ *Departamento de Álgebra, Geometría y Topología, \\ Facultad de Ciencias, Universidad de Málaga, \\ Campus Teatinos, E-29071 Málaga, Spain \\ ${ }^{\dagger}$ Departamento de Geometría y Topología, \\ Facultad de Ciencias, Universidad de Granada, \\ Campus Fuentenueva s/n, E-18071 Granada, Spain
}

\begin{abstract}
Recently, the old notion of causal boundary for a spacetime $V$ has been redefined consistently. The computation of this boundary $\partial V$ on any standard conformally stationary spacetime $V=\mathbb{R} \times M$, suggests a natural compactification $M_{B}$ associated to any Riemannian metric on $M$ or, more generally, to any Finslerian one. The corresponding boundary $\partial_{B} M$ is constructed in terms of Busemann-type functions. Roughly, $\partial_{B} M$ represents the set of all the directions in $M$ including both, asymptotic and "finite" (or "incomplete") directions.

This Busemann boundary $\partial_{B} M$ is related to two classical boundaries: the Cauchy boundary $\partial_{C} M$ and the Gromov boundary $\partial_{G} M$. In a natural way $\partial_{C} M \subset \partial_{B} M \subset \partial_{G} M$, but the topology in $\partial_{B} M$ is coarser than the others. Strict coarseness reveals some remarkable possibilities - in the Riemannian case, either $\partial_{C} M$ is not locally compact or $\partial_{G} M$ contains points which cannot be reached as limits of ray-like curves in $M$.

In the non-reversible Finslerian case, there exists always a second boundary associated to the reverse metric, and many additional subtleties appear. The spacetime viewpoint interprets the asymmetries between the two Busemann boundaries, $\partial_{B}^{+} M\left(\equiv \partial_{B} M\right), \partial_{B}^{-} M$, and this yields natural relations between some of their points.

Our aims are: (1) to study the subtleties of both, the Cauchy boundary for any generalized (possibly non-symmetric) distance and the Gromov compactification for any (possibly incomplete) Finsler manifold, (2) to introduce the new Busemann compactification $M_{B}$, relating it with the previous two completions, and (3) to give a full description of the causal boundary $\partial V$ of any standard conformally stationary spacetime.
\end{abstract}




\section{Contents}

1 Introduction 3

2 Preliminaries $\mathbf{1 0}$

2.1 Spacetimes and c-boundaries . . . . . . . . . . . . . 10

2.2 Finsler manifolds . . . . . . . . . . . . . . . . . . . . . 15

2.3 Conformally stationary spacetimes $\ldots \ldots \ldots \ldots \ldots$

3 Cauchy completion of a generalized metric space 17

3.1 Basic definitions . . . . . . . . . . . . . . 17

3.2 Forward and backward Cauchy boundaries as point sets . . . . . . 18

3.2.1 Cauchy sequences and completions. . . . . . . . . . 18

3.2.2 Alternative Cauchy sequences yield the same completions . . 21

3.2.3 The symmetrized Cauchy boundary . . . . . . . . . . 23

3.3 Quasi-distance and topology on the Cauchy completions . . . . . 2 25

3.4 Relating $M_{C}^{+}$and $M_{C}^{-}$through the extended quasi-distance . . . 29

4 Riemannian Gromov and Busemann completions 31

4.1 Gromov completion . . . . . . . . . . . . . . . . . 32

4.2 Busemann completion as a point set . . . . . . . . 39

5 Finslerian completions 42

5.1 Gromov completions for the non-symmetric case . . . . . . . . 43

5.2 Busemann completions . . . . . . . . . . . . . . . . 48

5.2.1 Busemann completion as a point set . . . . . . . . 48

5.2 .2 Chronological topology on $M_{B}^{+} \ldots \ldots \ldots \ldots \ldots$

5.2.3 The inclusion of $M_{C}^{+}$in $M_{B}^{+}$from the topological viewpoint . 51

5.2.4 Compactness of the Busemann completion . . . . . . . . . 54

5.2.5 The backward Busemann completion . . . . . . . . . . 555

5.3 Chronological topology vs Gromov topology . . . . . . . . . . 555

5.3.1 The inclusion of $M_{B}^{+}$in $M_{G}^{+}$from the topological viewpoint . 56

5.3 .2 The case of separating topologies . . . . . . . . . . 56

5.3.3 Topologies with unique limits of sequences . . . . . . . . 58

5.3 .4 Main results . . . . . . . . . . . . . . . . . . . 599

5.4 Proof of Theorem 1.1. . . . . . . . . . . . . . . . 61

6 C-boundary of standard stationary spacetimes 61

6.1 Chronological relations and Lipschitzian functions . . . . . . . . . 63

6.2 Future and past c-boundaries as point sets . . . . . . . . . . 64 64

6.3 The (total) c-boundary as a point set . . . . . . . . . 66 6 6

6.4 Causality of the c-boundary . . . . . . . . . . . . 71

6.5 Topology of the partial boundaries and the c-boundary . . . . . . . 772

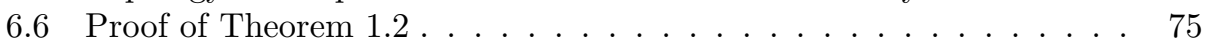




\section{Introduction}

In Differential Geometry there are quite a few boundaries which can be attached to a space, depending on the problem one would like to study. For a Riemannian manifold $(M, g)$, when $g$ is incomplete the Cauchy completion yields a simple boundary. When $g$ is complete, Gromov introduced a general compactification by using quotients of Lipschitz functions 17. Such a compactification coincides with Eberlein and O'Neill's one for a Hadamard manifold, which defines the boundary points as Busemann functions associated to rays, up to additive constants, and uses the cone topology [9. This construction can be extended to more general spaces (as the CAT(0) ones), but it was not conceived for an arbitrary Riemannian manifold. Among the different boundaries in Lorentzian Geometry (Schmidt's bundle boundary, Geroch's geodesic boundary, Penrose's conformal boundary...), the so-called causal boundary (or c-boundary for short) becomes especially interesting. The c-boundary was introduced by Geroch, Kronheimer and Penrose 14 by using a conformally invariant construction, which is explicitly intrinsic and systematic (in advantage with the conformal boundary, widely used in Mathematical Relativity). But some problems about its consistency originated a long sequence of redefinitions of this boundary (see [30] for a critical review). Recently, with the additional stimulus of finding a general boundary for the AdS-CFT correspondence, the notions of c-boundary and c-completion have been widely developed [18, 19, 26, 10, 30, and the recent detailed study in [12] justifies that a satisfactory definition is available now. Moreover, several new ideas have been introduced for the computation of the cboundary (or several non-problematic elements of it) in relevant cases [1, 11, 13, 20.

In this article we carry out a systematic study of the c-boundary of a natural class of spacetimes, the (conformally) stationary ones. However, our motivation is not only to compute this boundary for a remarkable class of spacetimes, but also the revision of other classical boundaries in Differential Geometry - which turn out to be related with the causal one. More precisely, in order to describe the c-boundary of a standard stationary spacetime $V=\mathbb{R} \times M$, the space of, say, "diverging directions of curves on $M$ " appears in a natural way. These directions must be computed with a Riemannian metric $g$ in the particular case that the spacetime is static, and with a Finsler metric $F$ of Randers type (and its reverse metric $F^{\text {rev }}$ ) in the general stationary case. Such directions are computed from Busemann-type functions constructed for arbitrary curves of bounded velocity, and its topology is naturally defined from the chronological topology for any c-completion. Of course, in the particular case that $(M, g)$ is a Hadamard manifold, the boundary agrees with Eberlein and O'Neill's one. But, in general, one obtains a new compactification of both, any Riemannian manifold and any Randers manifold, the latter trivially extensible to any Finslerian manifold. Then, it is natural to compare this new Busemann compactification of any Finslerian manifold with the classical Gromov and Cauchy completions. As far as we know, neither the Gromov compactification has been studied systematically for an arbitrary Finsler manifold, nor the Cauchy completion has been developed for a generalized distance, i.e. a non-necessarily symmetric distance (as the ones induced by Finsler metrics). As they present some 
non-evident properties (which will turn out to be essential for the c-boundary) our first aim will be to study both completions in some detail. Then, we will introduce the Busemann boundary of a Finsler manifold (with no reference to the causal boundary or Lorentzian Geometry) and compare it with the other two completions. Finally, with all these elements at hand, a detailed picture of the c-boundary of a standard stationary spacetime will be achieved.

The contents of the paper are organized as follows. The first subsection of Section 2 provides some generalities on the c-boundary construction for any strongly causal spacetime $V$. We emphasize some subtleties on this boundary $\partial V$ : (a) at a point set level, the c-boundary is constructed by making pairs of elements from the future and past causal (pre)boundaries $\hat{\partial} V, \check{\partial} V$ so that a point, say $P \in \hat{\partial} V$, may be paired with more than one point $F, F^{\prime} \in \partial \partial V$ yielding more than one pair $(P, F),\left(P, F^{\prime}\right)$ in $\partial V$, and (b) even when the points in $\hat{\partial} V, \partial \breve{V}$ yield only a single pair, the topology in $\partial V$ cannot be regarded as a plain topological quotient of these two boundaries. In fact, such involved cases originated a long list of redefinitions of the c-boundary - and, remarkably, they will occur even is stationary spacetimes. So, we introduce the notion of simple c-boundary for the cases when these subtle possibilities do not occur (Definition 2.4). One of the goals of the present article will be to prove that, under very mild hypotheses, the c-boundary of a stationary spacetime is simple. The other two subsections recall some properties of Finsler manifolds and conformally stationary spacetimes. The latter summarizes the approach in [8], and it allows to understand that the conformally invariant properties of a stationary spacetime are codified in a Finsler metric. After these preliminaries, the following topics are developed.

Section 3. The Cauchy completion of a non-necessarily symmetric distance is studied. Such distances are well-known since the old work by Zaustinsky [34, and our definitions agree with this author. We distinguish between generalized distances (which are close to the symmetric case, and appear in Finsler manifolds) and quasidistances (which will appear in the extensions of the generalized distances to the completions, and retain less properties), see Definition 3.1. Remark 3.2. We study the Cauchy completion of a generalized distance in some detail, because they contain some non-trivial subtleties, as the following:

(a) There are three types of natural Cauchy completions, the forward, the backward and the symmetrized ones (Definition 3.11 3.21. Proposition 3.22). Of course, the three completions coincide for any (symmetric) distance, but even the symmetrized boundary of a Finsler manifold may behave in a highly non-Riemannian way (Remark 3.23, Example 3.24).

(b) There are two natural definitions of (forward) Cauchy sequence. The usual one (Definition. 3.4 which agrees with $[3$, for example) is a straightforward generalization of the symmetric case. The alternative one (Definition 3.13 Proposition 3.14 seems more natural for the Cauchy completion (Proposition 3.16). These notions are not equivalent (Example 3.15), but they do yield equivalent Cauchy completions (Theorem 3.17, Remark 3.18), supporting the remainder of our approach.

(c) The generalized distance $d$ can be extended to the (say, forward) Cauchy 
completion $M_{C}^{+}$but, then, the extension $d_{Q}$ is only a quasi-distance (Proposition 3.25). This lies in the core of many subsequent difficulties; in fact, when $d_{Q}$ is a generalized distance on $M_{C}^{+}$then $M_{C}^{+}=M_{C}^{-}=M_{C}^{s}$ (Proposition 3.28). But for the quasi-distance $d_{Q}$, the topology associated to the open forward balls may be different to the one generated by the backward balls (and none of them is $T_{1}$ ). Moreover, only the latter topology ensures that $M_{C}^{+}$is truly a completion, i.e. any forward Cauchy sequence will be convergent in $M_{C}^{+}$with the topology generated by the backward balls (Remark 3.26. Convention 3.27. Theorem 3.29).

(d) It is even possible to extend further $d_{Q}$ (Proposition 3.32, yielding some relations between the forward and backward completions. Such relations will become quite natural under the viewpoint of the c-boundary (in fact, the asymmetries between $M_{C}^{+}$and $M_{C}^{-}$will reflect the asymmetries between the future and past directions in the spacetime). They support the notion of a generalized metric space with evenly pairing boundaries (Definition 3.34 see Figure 2), which will be useful for the computation of the c-boundary of a stationary spacetime $V$.

We emphasize that all the explained subtleties already appear in the simplest case of the generalized metric space associated to a Finsler metric of Randers type (the relevant case for the c-boundary of a stationary spacetime). So, the examples which exhibit these subtleties are chosen of this type.

Sections 4. 5. Here, Gromov and Busemann completions and boundaries are extended to arbitrary Finsler manifolds. For the convenience of the reader, some properties are studied first in Section 4 for the particular case of Riemannian manifolds (or somewhat more general spaces as reversible Finsler manifolds, see Convention 4.1). As far as we know, the Finslerian versions in Section 5 had not been considered before. Even though our study is independent of Lorentzian Geometry, our definitions are guided by the detailed correspondence between global Finsler properties and global conformally invariant Lorentzian elements (causal objects) developed in 8 . Some connections between Finsler and Lorentzian elements had been also introduced in [15] under a local viewpoint, and in [7] in the framework of variational methods.

For the Riemannian Gromov completion $M_{G}$, we distinguish between the proper Gromov boundary $\partial_{\mathcal{G}} M$, which contains "points at infinity", and the CauchyGromov one $\partial_{C G} M$. The latter includes the Cauchy boundary $\partial_{C} M$ and, roughly, it can be understood as a local compactification of $\partial_{C} M$ obtained by means of both, a coarser topology and the introduction of new boundary points which constitute the residual Gromov boundary $\partial_{R G} M$. In the Cauchy-Gromov completion $M_{C G}=M \cup \partial_{C G} M$, a Heine-Borel property holds; so, the natural condition to ensure that the Cauchy completion $M_{C}$ is topologically embedded in $M_{C G}$, is that $\partial_{C} M$ is locally compact (Corollary 4.13). All these properties are proved in the Riemannian case in Subsection 4.1, and then, only the specific difficulties of the Finslerian case are analyzed in Section 5.1. Essentially, these Finslerian difficulties involve three facts. First, the appropriate choices for notions such a Lipschitz function (Definition 5.2, Remark 5.3) or oriented-equicontinuity (Definition 5.10). Second, the existence of forward and backward completions $M_{G}^{+}, M_{G}^{-}$, which yield more possibilities to deal with (for example, the continuity of the inclusion of $M_{C}^{+}$ 
in $M_{G}^{+}$holds only under an additional hypothesis, see Lemma 5.5 and Remark 5.6. And, third, some specific technicalities must be analyzed now, as the necessity of a version of Arzela's theorem for oriented-equicontinuous curves (Theorem 5.15),

About the Busemann completion, it is inspired on two elements. On one hand (as a point set), in the commented Eberlein and O'Neill completion of Hadamard manifolds and, on the other (topologically), in the interpretation of the graphs of Busemann-type functions (on Riemannian and Randers-type Finsler manifolds) as points in the (future) causal completion of a stationary spacetime. Some properties of the Busemann completion for a Riemannian manifold had been already studied in [20, 11. Here, we present a systematic study, which includes all the Finslerian manifolds. For the convenience of the reader, we explain first the Busemann completion $M_{B}$ as a point set in the Riemannian case (Section 4.2). Such a $M_{B}$ (Definition 4.16 Remark 4.17) contains the points in Gromov's one $M_{G}$ which can be obtained from Busemann-type functions. Intuitively, this means that these points in $M_{G}$ can be "reached by curves asymptotically like a ray" (see Remarks 4.18, 5.41). So, in spite of their very different definitions, Gromov and Busemann completions agree in most cases as point sets. However, the cases when they do not coincide become also interesting, and the existence of such differences will be equivalent to the existence of differences at the topological level. For reasons of space, the study of the Busemann topology (or chr. topology) is postponed to the general Finlerian case. But the reader interested only in Riemannian Geometry will be able to reconstruct easily this part just by making some simplifications.

The Busemann completions $M_{B}^{ \pm}$in the general Finslerian case (or the somewhat more general spaces under Convention 5.1) are analyzed in Section 5.2. Its natural topology (the chronological topology) is adapted to the structure of the Busemann functions. It becomes somewhat technical, but it will admit natural interpretations from the spacetime viewpoint. The original space $M$ can be embedded naturally in $M_{B}^{ \pm}$, and these completions yield two compactifications of $M$. The Cauchy completion $M_{C}^{+}$is included in $M_{B}^{+}$so that $\partial_{B}^{+} M$ becomes the disjoint union of $\partial_{C}^{+} M$ ("incomplete finite directions") and the properly Busemann boundary $\partial_{\mathcal{B}}^{+} M$ ("asymptotic directions"). The inclusion at the topological level is also discussed, as in the Gromov case. The comparison between Gromov's and Busemann's completions is carried out in Section 5.3 As a point set, $M_{B}^{+}$is included in $M_{G}^{+}$and this inclusion may be strict (in particular, no point in $\partial_{B}^{+} M$ belongs to the residual Gromov boundary $\left.\partial_{R G}^{+} M\right)$. The chronological topology on $M_{B}^{+}$is coarser than the induced from $M_{G}^{+}$, but also yields a compactification of $M$. This is done at the price of being non-Hausdorff, and has a natural explanation: while Busemann boundary compactifies only among "asymptotically ray-like directions", Gromov one introduces additional points, not always reachable as the limit of some curve in $M$. Gromov completion has the nice Hausdorff property, but Busemann completion does not introduce points which may appear as spurious (Remark 5.41). Remarkably, both topologies agree when the Busemann completion $M_{B}^{+}$is Hausdorff, and this is equivalent to the equality of $M_{B}^{+}$and $M_{G}^{+}$as point sets (Theorem 5.39).

In conclusion, our study gives a precise description of the three boundaries for a Finslerian manifold, which becomes especially simple when the conditions which 
ensure both, $M_{B}^{+}=M_{G}^{+}$and the inclusion of $M_{C}^{+}$in $M_{B}^{+}$is an embedding, hold. The results in Sections 4, 5 are summarized in the following theorem:

Theorem 1.1 Let $(M, F)$ be any (connected) Finsler manifold with associated generalized distance $d$.

1. The (forward) Gromov completion $M_{G}^{+}$of $(M, d)$ endowed with the quotient of the pointwise convergence topology (see Definition 5.8) of $(M, d)$ is a compact metrizable topological space.

2. The (forward) Busemann completion $M_{B}^{+}$of $(M, d)$ endowed with the chronological topology (see Definitions 5.17. 5.18) is a possibly non-Hausdorff $T_{1}$ sequentially compact topological space.

The (possibly non-continuous) inclusion $i: M_{B}^{+} \rightarrow M_{G}^{+}$satisfies that $i^{-1}$ : $i\left(M_{B}^{+}\right) \rightarrow M_{B}^{+}$is always continuous (i.e., the chr. topology is coarser than the Gromov one).

The following statements are equivalent:

a) No sequence in $M_{B}^{+}$converges to more than one point in $\partial_{B}^{+} M$ with the chronological topology.

b) $M_{B}^{+}$is Hausdorff with the chronological topology.

c) $i: M_{B}^{+} \rightarrow M_{G}^{+}$is continuous (and thus, an embedding).

d) $M_{B}^{+}=M_{G}^{+}$both, as point sets and topologically.

e) $M_{B}^{+}$is equal to $M_{G}^{+}$as point sets.

3. The (forward) Cauchy completion $M_{C}^{+}$of $(M, d)$ is a set endowed with a quasidistance $d_{Q}$ (see Definition 3.1) which generates a natural $T_{0}$-topology (see Convention 3.27). Its relations with $M_{G}^{+}$and $M_{B}^{+}$are the following:

A) The natural map $j^{+}: M_{C}^{+} \rightarrow M_{G}^{+}$is injective, and it is continuous if and only if the backward topology on $M_{C}^{+}$is finer than the forward one (i.e. condition (a4') in Lemma 5.5 is satisfied). In particular, $j^{+}$is continuous if the quasi-distance $d_{Q}$ in $M_{C}^{+}$is a generalized distance (this property always happens in the Riemannian case).

The Cauchy-Gromov completion $M_{C G}^{+}$(Section 5.1, below Theorem 5.9) includes naturally the Cauchy one $M_{C}^{+}$as a point set, and it satisfies the Heine-Borel property (i.e., the closure in $M_{C G}^{+}$of any bounded set in $M$ is compact); in particular, it is locally compact.

Moreover, if $d_{Q}$ is a generalized distance and $M_{C}^{+}$is locally compact: a) $j^{+}$is an embedding, b) the Cauchy completion agrees with the bounded part of the Gromov one both, as a point set and topologically (there exists no residual Cauchy-Gromov boundary, i.e. $\left.\partial_{R G}^{+} M:=\partial_{C G}^{+} M \backslash \partial_{C}^{+} M=\emptyset\right)$.

B) Let $j_{B}^{+}: M_{C}^{+} \rightarrow M_{B}^{+}$obtained by restricting $j^{+}\left(\right.$as $\left.j^{+}\left(M_{C}^{+}\right) \subset M_{B}^{+}\right)$ and consider the natural topologies (chronological on $M_{B}^{+}$and the one in 
Convention 3.27 on $M_{C}^{+}$). Then, $j_{B}^{+}$is continuous if the backward topology generated by $d_{Q}$ on $M_{C}^{+}$is finer than the forward one. In particular, $j_{B}^{+}$is always continuous in the Riemannian case.

At any case, $M$ is naturally an open dense subset of $M_{G}^{+}, M_{B}^{+}$and $M_{C}^{+}$, each one endowed with its natural topology. So, the boundaries $\partial_{G}^{+} M$ and $\partial_{B}^{+} M$ are sequentially compact and these two boundaries coincide if and only if no pair of points in $\partial_{B}^{+} M$ is the limit of a single sequence in $M$.

Section 6. By using previous machinery, the c-boundary of a (standard) stationary spacetime $V$ is computed now. The results are summarized in Theorem 1.2 below (Figures 8 , 9 and 10 may be clarifying). But, previously, the following remarks are in order:

(a) First, we have to compute the future causal boundary $\hat{\partial} V$, composed by indecomposable past sets, IPs (plus the past causal boundary $\partial{ }^{2}$, composed by indecomposable future sets, IFs). This boundary will be endowed with Harris' chronological topology defined in [19]. At least when $M_{B}^{+}$is Hausdorff, $\hat{\partial} V$ has a plain structure: it is just a cone on the forward Busemann completion $\partial_{B}^{+} M$ (Proposition 6.10, Theorem 6.26). Moreover, the points of the symmetrized Cauchy boundary $\partial_{C}^{s} M$ yield timelike lines of the cone, and the other points of $\partial_{B}^{+} M$ yield lightlike lines. These results refine those in [11, 20, for the static case, and extend them to the stationary one.

(b) As we have already commented, some problems in the consistency of the notion of c-boundary $\partial V$ for a spacetime has persisted along almost three decades. They affected to: (i) the identification problem between points of $\hat{\partial} V$ and $\check{\partial} V$ (transformed in a pairing prescription by [26]), (ii) the minimal character of the c-boundary [10, 30] and (iii) its appropriate topology [10. The careful study by the authors in [12] gives an answer to these questions, and we follow this approach. Even though some of these subtleties had appeared in somewhat pathological spacetimes, we can find examples of all of them in standard stationary spacetimes (see, for example, Remark 6.21. with Examples 6.19, 6.20 about the questions (i) and (ii)). In particular, the S-relation, which relates pairs IP-IF and defines the ccompletion as a point set (see 2.1), (2.2) below) was trivial in the static case, but it may be much more complicated here (Remark 6.21). The subtleties relative to the topology become especially interesting, even in the static case, as the correct topological (and chronological) description of $\partial V$ requires the notion of Busemann completion $M_{B}$ for a Riemannian manifold, plus the identification of $M_{C}$ with a subset of $M_{B}$ (endowed with the topology induced by $M_{B}$ ). This is studied in detail in Sections 6.36.5. The refinement of the definition of c-boundary left as an open possibility in our study [12] (the distinction between causal boundary and properly causal boundary) does not apply here, as the c-boundary in the standard stationary case is always properly causal, Remark 6.12 .

(c) We also emphasize the remarkable role of the Cauchy boundaries $\partial_{C}^{ \pm} M$ in the computation of $\partial V$. If these boundaries where empty, no point in $\partial V$ would be timelike and $\partial V$ would be automatically globally hyperbolic. So, no pairings between points of $\hat{\partial} V$ and $\check{\partial} V$ would occur (i.e. all the pairs in $\partial V$ would be type 
$(P, \emptyset),(\emptyset, F)$ for some $P \in \hat{\partial} V$ or $F \in \check{\partial} V)$. Therefore, a mathematically elegant assumption of completeness for the generalized distance $d$ (as it is assumed in the study of the classical Gromov boundary) would spoil many beautiful possibilities for spacetimes. Moreover, we emphasize that the Busemann boundaries $\partial_{B}^{ \pm} M$ split in two parts, the proper Busemann boundary $\partial_{\mathcal{B}}^{ \pm} M$ and the Cauchy one $\partial_{C}^{ \pm} M$ (the latter with a topology which comes from the Busemann viewpoint, not from the viewpoint of metric spaces). Roughly, the former $\partial_{\mathcal{B}}^{+} M$ corresponds with asymptotic directions in $M$ and yields (most of) the lightlike part of $\hat{\partial} V$. The latter $\partial_{C}^{+} M$ corresponds with diverging directions at bounded distance in $M$, it yields the timelike points of $\partial V$ and some very particular lightlike ones.

Summing up:

Theorem 1.2 Let $(V, g)$ be a standard (conformally) stationary spacetime (see (2.4) and normalize $\Lambda \equiv 1$ with no loss of generality). Let $F^{ \pm}$be its associated Finsler metrics (or Fermat metrics, see (2.5)), and denote as $d^{ \pm}$the generalized distances associated to $F^{ \pm}$. Then, the c-boundary $\partial V$ has the following structure:

1. Point set:

(1A) The future (resp. past) c-boundary $\hat{\partial} V$ (resp. $\check{\partial} V$ ) is naturally a point set cone with base $\partial_{B}^{+} M$ (resp. $\left.\partial_{B}^{-} M\right)$ and apex $i^{+}$(resp. $\left.i^{-}\right)$.

(1B) A pair $(P, F) \in \partial V$ with $P \neq \emptyset$ satisfies that $P=P\left(b_{c}^{+}\right)$for some $c \in C^{+}(M)$ (see Proposition 6.6) and:

(a) If $b_{c}^{+} \equiv \infty$ then $P=V, F=\emptyset$.

(b) If $b_{c}^{+} \in \mathcal{B}^{+}(M)\left(\equiv \mathbb{R} \times \partial_{\mathcal{B}}^{+} M\right.$, see (6.4) ) then $F=\emptyset$.

(c) If $b_{c}^{+} \in B^{+}(M) \backslash \mathcal{B}^{+}(M)$, then $b_{c}^{+}=d_{p}^{+}$with $p=\left(\Omega^{+}, x^{+}\right) \in \mathbb{R} \times$ $\partial_{C}^{+} M$ (see (5.6)), $P=P\left(d_{p}^{+}\right)$and $F \subset F\left(d_{p}^{-}\right)$. In this case, there are two exclusive possibilities:

(c1) either $F=\emptyset$,

(c2) or $F=F\left(d_{p^{\prime}}^{-}\right)$with $p^{\prime}=\left(\Omega^{-}, x^{-}\right) \in \mathbb{R} \times \partial_{C}^{-} M$ and satisfying $\Omega^{-}-\Omega^{+}=d^{+}\left(x^{+}, x^{-}\right)$(in this case, $p^{\prime}$ is not necessarily unique).

Moreover, if $x \in \partial_{C}^{s} M$, then $p^{\prime}=p, \uparrow P=F\left(d_{p}^{-}\right)$and $P$ is univocally S-related with $F=F\left(d_{p}^{+}\right)$.

A dual result holds for pairs $(P, F)$ with $F \neq \emptyset$. So, the total c-boundary is the disjoint union of lines $L(P, F)$ (see Definition 6.17).

When $\partial V$ is simple as a point set (see Definition 2.4: in particular this happens when $\left(M, d^{+}\right)$has a forward or backward evenly pairing boundary, Definition 3.34), then $\partial V$ it is the quotient set $\hat{\partial} V \cup_{d} \partial \check{V} V / \sim_{S}$ of the partial boundaries $\partial V$, $\mathrm{V}$ under the S-relation.

2. Causality: For each pair $(P, F) \in \partial V$, the line $L(P, F)$ is timelike if $P=$ $P\left(d_{p}^{+}\right)$and $F=F\left(d_{p}^{-}\right)$for some $p \in \mathbb{R} \times \partial_{C}^{s} M$, horismotic if either $P$ or $F$ are empty and locally horismotic otherwise (recall Definition 6.22). 


\section{Topology:}

(3A) If $M_{B}^{+}$(resp. $M_{B}^{-}$) is Hausdorff, the future (resp. past) causal boundary has the structure of a (topological) cone (Definition 6.9) with base $\partial_{B}^{+} M$ (resp. $\left.\partial_{B}^{-} M\right)$ and apex $i^{+}$(resp. $\left.i^{-}\right)$.

(3B) If $M_{C}^{s}$ is locally compact and $d_{Q}^{+}$is a generalized distance, then $\bar{V}$ is simple (see Definition 2.4) and so, it coincides with the quotient topological space $\hat{V} \cup_{d} \check{V} / \sim_{S}$ of the partial completions $\hat{V}$ and $\check{V}$ under the S-relation.

Summarizing, if $M_{C}^{s}$ is locally compact, $d_{Q}^{+}$is a generalized distance and $M_{B}^{ \pm}$is Hausdorff, $\partial V$ coincides with the quotient topological space $\left(\hat{\partial} V \cup_{d} \check{\partial} V\right) / \sim_{S}$, where $\hat{\partial} V$ and $\check{\partial} V$ have the structure of cones with bases $\partial_{B}^{+} M, \partial_{B}^{-} M$ and apexes $i^{+}, i^{-}$, resp.

Remark 1.3 Previous theorem can be extended to the corresponding causal completions in the obvious natural way (replacing the boundary of each type by the corresponding completion), at all the levels, i.e., as a pointset, causally and topologically.

\section{Preliminaries}

\subsection{Spacetimes and c-boundaries}

We will use typical background and terminology in Lorentzian Geometry as in 4, 27, 29. A spacetime will be a time-oriented connected smooth Lorentzian manifold $(V, g)$ (the time-orientation, and so, the choice of a future cone at each tangent space, will be assumed implicitly) of signature $(-,+, \ldots,+)$. A tangent vector $v \in T_{p} V, p \in V$ is called timelike (resp. lightlike; causal) if $g(v, v)<0$ (resp. $g(v, v)=0, v \neq 0 ; v$ is either timelike or lightlike); null vectors include both, the lightlike ones and the 0 vector. A causal vector is called future or past-directed if it belongs to the future or past cone. Accordingly, a smooth curve $\gamma: I \rightarrow V$ ( $I$ real interval) is called timelike, lightlike, causal and future or past-directed if so is $\dot{\gamma}(s)$ for all $s \in I$.

Two events $p, q \in V$ are chronologically related $p \ll q$ (resp. causally related $p \leq q$ ) if there exists some future-directed timelike (resp. either future-directed causal or constant) curve from $p$ to $q$. If $p \neq q$ and $p \leq q$ but $p \nless q$, then $p$ is said horismotically related to $q$. The chronological past (resp. future) of $p, I^{-}(p)$ (resp. $\left.I^{+}(p)\right)$ is defined as:

$$
I^{-}(p)=\{q \in V: q \ll p\} \quad\left(\text { resp. } I^{+}(p)=\{q \in V: p \ll q\}\right) .
$$

In what follows, we will be especially interested in the chronological past $I^{-}[\gamma]=$ $\cup_{s \in I} I^{-}(\gamma(s))$ (resp. future $I^{+}[\gamma]=\cup_{s \in I} I^{+}(\gamma(s))$ ) of any future-directed (resp. past-directed) timelike curve $\gamma: I \rightarrow V$. 
Remark 2.1 Usually, in the literature a causal curve $\gamma$ with a compact domain $I=[a, b]$ is allowed to be piecewise smooth, that is, it admits a partition $a=t_{0}<$ $t_{1}<\ldots<t_{n}=b$ such that: (i) the restriction of $\gamma$ to each subinterval $\left[t_{j}, t_{j+1}\right]$ is smooth and causal, and (ii) the left and right velocities of $\gamma$ at each break $t_{j}$ lie in the same cone. This is technically convenient, even though typically it does not represent a true increment of generality (for example, in order to define the relations $\ll, \leq$ ), because any such curve admits a variation through smooth causal ones with the same endpoints.

Here, we will use typically, say, future-directed timelike curves defined on a halfopen interval $I=[a, b)$, which cannot be continuously extended to $b$. One can assume also that such a curve $\rho$ is piecewise smooth in the sense that there exists a strictly increasing sequence $\left\{t_{j}\right\} \nearrow b$ included in $I$ such that conditions (i) and (ii) above hold. In fact, on one hand, all the techniques to be used here will work trivially in this alternative case. On the other, for any such a piecewise smooth curve $\rho$ there exists a smooth future-directed causal curve $\tilde{\rho}$ arbitrarily close to $\rho$ such that $I^{-}[\tilde{\rho}]=I^{-}[\rho]$ (see for example [13, Sect. 3.3])

The c-completion of spacetimes is constructed by adding ideal points to the spacetime in such a way that any timelike curve acquires some endpoint in the new space 14. To formalize this construction, which will be conformally invariant and applicable to strongly causal spacetimes, previously we need to introduce some basic notions.

A non-empty subset $P \subset V$ is called a past set if it coincides with its past; i.e. $P=I^{-}[P]:=\{p \in V: p \ll q$ for some $q \in P\}$. The common past of $S \subset V$ is defined by $\downarrow S:=I^{-}[\{p \in V: \quad p \ll q \forall q \in S\}]$. In particular, the past and common past sets must be open. A past set that cannot be written as the union of two proper subsets, both of which are also past sets, is called indecomposable past set, IP. An IP which does coincide with the past of some point of the spacetime $P=I^{-}(p), p \in V$ is called proper indecomposable past set, PIP. Otherwise, $P=I^{-}[\gamma]$ for some inextendible future-directed timelike curve $\gamma$, and it is called terminal indecomposable past set, TIP. The dual notions of future set, common future, IF, TIF and PIF, are obtained just by interchanging the roles of past and future in previous definitions.

To construct a future and a past causal completion, first we have to identify each event $p \in V$ with its PIP, $I^{-}(p)$, and PIF, $I^{+}(p)$. This is possible in any distinguishing spacetime, that is, a spacetime which satisfies that two distinct events $p, q$ have distinct chronological futures and pasts $\left(p \neq q \Rightarrow I^{ \pm}(p) \neq I^{ \pm}(q)\right)$. In order to obtain consistent topologies in the completions, we will define the c-completion for a somewhat more restrictive class of spacetimes, the strongly causal ones. These are characterized by the fact that the PIPs and PIFs constitute a sub-basis for the topology of the manifold $V$.

Now, every event $p \in V$ can be identified with its PIP, $I^{-}(p)$. So, the future

\footnotetext{
${ }^{1}$ If $\rho$ were allowed to be continuously extensible to $b$ then the extension may be non-differentiable at $b$. However, it would be locally Lipschitzian and $H^{1}$ (see [6] Appendix A]), and any futuredirected (smooth) causal curve $\tilde{\rho}$ with endpoint $\rho(b)$ would satisfy $I^{-}[\tilde{\rho}]=I^{-}(\rho(b))=I^{-}[\rho]$.
} 
causal boundary $\hat{\partial} V$ of $V$ is defined as the set of all the TIPs in $V$, and the future causal completion $\hat{V}$ becomes the set of all the IPs:

$$
V \equiv \mathrm{PIPs}, \quad \hat{\partial} V \equiv \mathrm{TIPs}, \quad \hat{V} \equiv \operatorname{IPs} .
$$

Analogously, each $p \in V$ can be identified with its PIF, $I^{+}(p)$. The past causal boundary $\partial V$ of $V$ is defined as the set of all the TIFs in $V$, and the past causal completion $\check{V}$ is the set of all the IFs:

$$
V \equiv \mathrm{PIFs}, \quad \check{\partial} V \equiv \mathrm{TIFs}, \quad \check{V} \equiv \mathrm{IFs} .
$$

For the (total) causal boundary, the so-called S-relation comes into play [31, 32]. Denote $\hat{V}_{\emptyset}=\hat{V} \cup\{\emptyset\}$ (resp. $\left.\check{V}_{\emptyset}=\check{V} \cup\{\emptyset\}\right)$. The S-relation $\sim_{S}$ is defined in $\hat{V}_{\emptyset} \times \breve{V}_{\emptyset}$ as follows. If $(P, F) \in \hat{V} \times \check{V}$,

$$
P \sim_{S} F \Longleftrightarrow \begin{cases}F & \text { is included and is a maximal IF in } \uparrow P \\ P & \text { is included and is a maximal IP in } \downarrow F .\end{cases}
$$

Recall that, as proved by Szabados [31, $I^{-}(p) \sim_{S} I^{+}(p)$ for all $p \in V$, and these are the unique S-relations (according to our definition (2.1)) which involve proper indecomposable sets. For $(P, F) \in \hat{V}_{\emptyset} \times \check{V}_{\emptyset}$, with $(P, F) \neq(\emptyset, \emptyset)$, we also put

$$
P \sim_{S} \emptyset, \quad\left(\operatorname{resp} . \emptyset \sim_{S} F\right)
$$

if $P$ (resp. $F$ ) is a (non-empty, necessarily terminal) indecomposable set and it is not S-related by 2.1) to any other indecomposable set. Now, we can introduce the notion of c-completion, according to [12]:

Definition 2.2 As a point set, the c-completion $\bar{V}$ of a strongly causal spacetime $V$ is formed by all the pairs $(P, F) \in \hat{V}_{\emptyset} \times \check{V}_{\emptyset}$ such that $P \sim_{S} F$. The c-boundary $\partial V$ is defined as $\partial V:=\bar{V} \backslash V$, where $V \equiv\left\{\left(I^{-}(p), I^{+}(p)\right): p \in V\right\}$.

The chronological relation of the spacetime is extended to the completion in the following way. We say that $(P, F),\left(P^{\prime}, F^{\prime}\right) \in \bar{V}$ are chronologically related, $(P, F) \ll\left(P^{\prime}, F^{\prime}\right)$, if $F \cap P^{\prime} \neq \emptyset$.

Finally, the topology of the spacetime is also extended to the completion by means of the so-called chronological topology (chr. topology, for short). The motivation for this topology comes from the following observation [19]. The natural topology of $V$ can be characterized by a limit operator $\hat{L}$ which determines the possible limits for any sequence $\sigma=\left\{x_{n}\right\}$ in $V$. More precisely, we write $x \in \hat{L}(\sigma)$ if and only if

(a) for all $y \ll x$, for all sufficiently large $n, y \ll x_{n}$, and

(b) for any $x^{\prime}$ with $I^{-}(x) \subset I^{-}\left(x^{\prime}\right)$, if for all $y \ll x^{\prime}$, there is infinitely many $n$ 's with $y \ll x_{n}$, then $x=x^{\prime}$. 
The operator $\hat{L}$ can be rewritten in terms of IP's and, then, it can be applied to any sequence in $\hat{V}$. Moreover, it can be used to define the natural (future) chr. topology on $\hat{V}$ by imposing that a subset $C \subset \hat{V}$ is closed if and only if $\hat{L}(\sigma) \subset C$ for any sequence $\sigma \subset C$ (recall that, now, the limit operator $\hat{L}$ determines the convergence of sequences in an indirect way [19, p. 562]). A dual construction can be carried out with a limit operator $\check{L}$ which defines the (past) chr. topology on $\breve{V}$. For the chr. topology on the (total) causal boundary $\bar{V}$, a similar construction is done by using a single operator $L$ constructed from the previous two ones $\hat{L}, \check{L}$.

Rigorously, the limit operator $L$ for sequences in $\bar{V}$ is defined as follows: given a sequence $\sigma=\left\{\left(P_{n}, F_{n}\right)\right\} \subset \bar{V}$ and $(P, F) \in \bar{V}$,

$$
(P, F) \in L(\sigma) \Longleftrightarrow \text { if } P \neq \emptyset, P \in \hat{L}\left(P_{n}\right) \text { and if } F \neq \emptyset, F \in \check{L}\left(\left\{F_{n}\right\}\right) .
$$

where 2

$$
\begin{aligned}
& \hat{L}\left(\left\{P_{n}\right\}\right):=\left\{P^{\prime} \in \hat{V}: P^{\prime} \subset \operatorname{LI}\left(\left\{P_{n}\right\}\right) \text { and } P^{\prime} \text { is a maximal IP into } \operatorname{LS}\left(\left\{P_{n}\right\}\right)\right\} \\
& \check{L}\left(\left\{F_{n}\right\}\right):=\left\{F^{\prime} \in \hat{V}: F^{\prime} \subset \operatorname{LI}\left(\left\{F_{n}\right\}\right) \text { and } F^{\prime} \text { is a maximal IF into } \operatorname{LS}\left(\left\{F_{n}\right\}\right)\right\} .
\end{aligned}
$$

Then, the operator $L$ defines the closed sets for the chronological topology on $\bar{V}$ as those subsets $C \subset \bar{V}$ such that $L(\sigma) \subset C$ for any sequence $\sigma \subset C$. The chr. topologies on the partial completions $\hat{V}, \check{V}$ are expressible analogously in terms of $\hat{L}, \breve{L}$, respectively.

Remark 2.3 We remark the following assertions about the chronological topology:

(1) Clearly, if $(P, F) \in L\left(\left\{\left(P_{n}, F_{n}\right)\right\}\right)$ then $\left\{\left(P_{n}, F_{n}\right)\right\}$ converges to $(P, F)$. When the converse happens, $L$ is called of first order (see the discussion [12, Section 3.6]).

(2) Given a pair $(P, F) \in \partial V$, any timelike curve defining $P$ (or $F$ ) converges to $(P, F)$ with the chronological topology (see [12, Th. 3.27]).

These definitions for the c-boundary construction involve some particular subtleties, which are essentially associated to the following two facts: an IP (or IF) does not determine a unique pair in the c-boundary; and the topology does not always agree with the S-relation, in the sense that

$$
P \in \hat{L}\left(P_{n}\right) \Leftrightarrow F \in \check{L}\left(F_{n}\right) .
$$

This makes natural to distinguish the following special cases:

Definition 2.4 A spacetime $V$ has a c-completion $\bar{V}$ which is simple as a point set if each TIP (resp. each TIF) determines a unique pair in $\partial V$.

Moreover, the c-completion is simple if it is simple as a point set and also topologically, i.e. $(P, F) \in L\left(P_{n}, F_{n}\right)$ holds when either $P \in \hat{L}\left(\left\{P_{n}\right\}\right)$ or $F \in \check{L}\left(\left\{F_{n}\right\}\right)$.

Observe that the condition of being simple as a point set is, indeed, a condition only on $\partial V$ i.e., the notion of being simple as a point set makes sense for $\partial V$ - this

\footnotetext{
${ }^{2} \mathrm{By}$ LI and LS we mean the usual inferior and superior limits of sets: i.e. $\operatorname{LI}\left(\left\{A_{n}\right\}\right) \equiv$ $\lim \inf \left(A_{n}\right):=\cup_{n=1}^{\infty} \cap_{k=n}^{\infty} A_{k}$ and $\operatorname{LS}\left(\left\{A_{n}\right\}\right) \equiv \lim \sup \left(A_{n}\right):=\cap_{n=1}^{\infty} \cup_{k=n}^{\infty} A_{k}$.
} 
obeys to the original idea by Geroch, Kronheimer and Penrose [14 of regarding the causal boundary as a quotient of $\hat{\partial} V$ and $\check{\partial} V$. Nevertheless, the simplicity at the topological level involves also the spacetime $V$. Moreover, from the definition of $L$, when $P \in \hat{L}\left(\left\{P_{n}\right\}\right)$ and $F \in \check{L}\left(\left\{F_{n}\right\}\right)$ one has $(P, F) \in L\left(P_{n}, F_{n}\right)$ always, and if, say, $P$ is the empty set, then only the second of the previous conditions is required (the first one would not make sense, as the empty set is never an element of $\hat{L}\left(P_{n}\right)$ ). So, the topological simplicity must be checked only when $P \neq \emptyset \neq F$.

When $\bar{V}$ is simple as a point set then one can consider the disjoint union $\hat{V} \cup_{d} \check{V}$ and the quotient $\hat{V} \cup_{d} \check{V} / \sim_{S}$ obtained by identifying each TIP $P$ (resp. IF $F$ ) with itself and, eventually, with the unique $F$ such that $P \sim_{S} F$. Clearly, $\hat{V} \cup_{d} \tilde{V}$ inherits a natural topology such that $\hat{V}$ and $\check{V}$ are its connected parts and, then, $\hat{V} \cup_{d} \check{V} / \sim_{S}$ is also regarded as a quotient topological space.

Proposition 2.5 Let $(V, g)$ be a spacetime:

(1) If the chronological topology associated to the c-completion $\bar{V}$ is Hausdorff, then the c-completion $\bar{V}$ is simple as a point set.

(2) If $\bar{V}$ is simple, then the the c-completion is naturally homeomorphic to $\hat{V} \cup_{d} \tilde{V} / \sim_{S}$ through the mapping:

$$
p: \bar{V} \rightarrow \hat{V} \cup_{d} \check{V} / \sim S, \quad p(P, F):=\left\{\begin{array}{ll}
{[P]} & \text { if } P \neq \emptyset \\
{[F]} & \text { if } F \neq \emptyset
\end{array} .\right.
$$

Proof. (1) From Remark $2.3(2)$, any timelike curve defining a TIP $P \neq \emptyset$ converges to any pair of the form $(\bar{P}, F) \in \bar{V}$ with the chronological topology. Then, as $\bar{V}$ is Hausdorff, $F$ is unique.

(2) First, let us characterize the closed sets in $\hat{V} \cup_{d} \check{V} / \sim S$. As the natural projection $\pi: \hat{V} \cup_{d} \check{V} \rightarrow \hat{V} \cup_{d} \check{V} / \sim S$ is continuous, and for any set $C \in \hat{V} \cup_{d} \check{V} / \sim S$

$$
\pi^{-1}(C)=\hat{C} \cup_{d} \check{C} \subset \hat{V} \cup_{d} \check{V}
$$

for some $\hat{C} \subset \hat{V}, \check{C} \subset \check{V}$, we have that $C$ is closed if and only if so are $\hat{C}$ and $\check{C}$.

Now, recall that $p$ in 2.3 is well defined and bijective because of the simplicity as a point set of $\bar{V}$. To check that $p$ is continuous, let $C$ be a closed subset of $\bar{V} \rightarrow \hat{V} \cup_{d} \tilde{V} / \sim S$ and assume by contradiction that $p^{-1}(C)$ is not closed in $\bar{V}$. Then, there exist a sequence $\left\{\left(P_{n}, F_{n}\right)\right\} \subset p^{-1}(C)$ and some $(P, F) \notin p^{-1}(C)$ such that $(P, F) \in L\left(P_{n}, F_{n}\right)$. From the definition of the limit operator $L$, if $P \neq \emptyset$ (otherwise, reason with $F \neq \emptyset)$ then $P \in \hat{L}\left(P_{n}\right)$. Nevertheless, this is a contradiction with the closedness of $C$ (and then $\hat{C}$ ), because by hypothesis $\left\{P_{n}\right\} \subset \hat{C}$ but $P \notin \hat{C}$.

For the continuity of $p^{-1}$, let $C$ be now a closed set in $\bar{V}$ and assume by contradiction that $p(C)$ is not closed in $\hat{V} \cup_{d} \check{V} / \sim_{S}$. That is, $\pi^{-1}(p(C))=p(\hat{C}) \cup_{d} p(\check{C})$ is not closed in $\hat{V} \cup_{d} \check{V}$ and, with no loss of generality, assume that $p(\hat{C})$ is not closed in $\hat{V}$. Then, there exist a sequence $\left\{P_{n}\right\} \in p(\hat{C})$ and some $P \notin p(\hat{C})$ such that $P \in \hat{L}\left(P_{n}\right)$. As $\bar{V}$ is (topologically) simple, $(P, F) \in L\left(P_{n}, F_{n}\right)$, with $\left(P_{n}, F_{n}\right) \in C$ and $(P, F) \notin C$, in contradiction with the closed character of $C$. 


\section{$2.2 \quad$ Finsler manifolds}

In this section, some basic elements of Finsler manifolds are outlined (for a general background, see for example [3]).

Definition 2.6 A Finsler metric on a smooth manifold $M$ is a non-negative function $F$ defined on the tangent bundle $T M$ which is continuous on $T M, C^{\infty}$ on $T M \backslash\{0\}$, it vanishes only on the zero section, it is fiberwise positively homogeneous of degree one (i.e. $F(x, \lambda v)=\lambda F(x, v) \forall x \in M, v \in T_{x} M$ and $\lambda>0$ ), and $F^{2}$ is fiberwise strongly convex (i.e. the matrix $\left[\partial^{2} F^{2} / \partial v^{i} \partial v^{j}(x, v)\right]$ is positive definite for any $(x, v) \in T M \backslash\{0\}$, where the $v^{i}$ 's denote linear coordinates in $\left.T_{x} M\right)$.

A Finsler manifold is a pair $(M, F)$ composed by a smooth manifold $M$ and a Finsler metric $F$.

Notice that, in general, a Finsler metric $F$ is not reversible, i.e. it may happen that $F(x, v) \neq F(x,-v)$ for some $(x, v)$. The reversed Finsler metric $F^{\text {rev }}$ of $F$ is then

$$
F^{\mathrm{rev}}(x, v):=F(x,-v) \quad \text { for all }(x, v) .
$$

In what follows, the classical notation above will be simplified by putting $F(v)$ $(v \in T M)$ instead of $F(x, v)\left(x \in M, v \in T_{x} M\right)$. The length of a piecewise smooth curve $c:\left[s_{0}, s_{1}\right] \rightarrow M$ with respect to a Finsler metric $F$ on $M$ is given by:

$$
\operatorname{length}_{F}(c):=\int_{s_{0}}^{s_{1}} F(\dot{c}(s)) d s .
$$

Because of non-reversibility, this length is not invariant under reparametrizations which change the orientation. However, one still has:

Proposition 2.7 For any Finsler manifold $(M, F)$ the map $d_{F}: M \times M \rightarrow \mathbb{R}$

$$
d_{F}(x, y):=\inf _{c \in C(x, y)} \operatorname{length}_{F}(c) \geq 0,
$$

where $C(x, y)$ is the set of piecewise smooth curves starting at $x$ and ending at $y$, satisfies all the axioms of a distance on $M$ but symmetry (i.e., $d_{F}(x, y) \neq d_{F}(y, x)$, in general).

The conclusion can be restated by saying that $\left(M, d_{F}\right)$ is a generalized metric space according to Defn. 3.1 below (see Remark 3.3).

A Randers metric on $M$ is a Finsler metric $F$ which can be written as $F(v)=$ $\sqrt{g_{0}(v, v)+\omega(v)^{2}}+\omega(v)$ for some Riemannian metric $g_{0}$ and 1-form $\omega$ on $M$. Randers metrics constitute a typical class of non-reversible Finsler metrics, and will appear naturally in standard stationary spacetimes.

\subsection{Conformally stationary spacetimes}

A conformally stationary (resp. stationary) spacetime is a spacetime $(V, g)$ which admits a timelike conformal-Killing (resp. Killing) vector field $K$; in this case, the 
vector field $K$ is also called conformally stationary (resp. stationary). ( $V, g)$ is standard conformally stationary if it can be written as:

$$
V=\mathbb{R} \times M \quad \text { and } \quad g=\Lambda\left(-d t^{2}+\pi^{*} \omega \otimes d t+d t \otimes \pi^{*} \omega+\pi^{*} g_{0}\right),
$$

where $\left(M, g_{0}\right)$ is a Riemannian manifold, $\omega$ a 1 -form on $M, \Lambda$ a positive function on $V$ and $t: \mathbb{R} \times M \rightarrow \mathbb{R}, \pi: \mathbb{R} \times M \rightarrow M$ the natural projections. Locally, any conformally stationary spacetime looks like a standard one with $K=\partial_{t}$. If $\Lambda$ in (2.4) is independent of the $t \in \mathbb{R}$ coordinate then $(V, g)$ is called standard stationary.

Along this work, we will assume two harmless simplifications. First, the conformally stationary vector field $K$ will be always unitary $(g(K, K) \equiv-1)$ and, thus, stationary. The reason is that we will be interested only in conformally invariant elements (as so is the c-boundary) and, thus, we can change the original metric $g$ by $g^{*}=-g / g(K, K)$ with no loss of generality.

The second one is to consider always the standard stationary case i.e., globally the expression (2.4) (with $\Lambda \equiv 1$ ). This is a natural simplification because a recent result in 23. shows that a stationary spacetime $V$ is standard if and only if: (i) it is distinguishing, and (ii) admits a complete stationary vector field $K$. As we have seen, condition (i) is necessary to deal with the c-boundary (in fact, it is less restrictive than strong causality). Condition (ii) is not fulfilled in general: typically, one can put some holes in a standard stationary spacetime and both, the standard character and the completeness of $K$ are dropped. However, in such an example, the spacetime would admit an open conformal embedding in the original standard stationary spacetime, (and, for example, the results in [12] which relate the causal and conformal boundaries may be applicable). So, the restriction to the standard case allows us to focus on the specific problems of the c-boundary for a stationary spacetime.

As we will see along this paper, the structure of the c-boundary for these spacetimes is closely related to properties of two Finsler metrics $F^{+}, F^{-}$of Randers type on the spatial part $M$. These metrics (or Fermat metrics) are defined in terms of $g_{0}$ and $\omega($ for $\Lambda \equiv 1)$; concretely,

$$
F^{ \pm}(v)=\sqrt{g_{0}(v, v)+\omega(v)^{2}} \pm \omega(v) \quad \forall v \in T M .
$$

We will put simply $F=F^{+}$, and then, $F^{\text {rev }}=F^{-}$. It is worth pointing out that the same standard stationary spacetime can be decomposed as standard stationary in more than one way (with different $g_{0}$ and $\omega$ ). Then, all the Fermat metrics associated to some decomposition will share those properties which are associated to the geometry of the spacetime $V$. These properties include the possible global hyperbolicity or causal simplicity of the spacetime (see [8]). 


\section{Cauchy completion of a generalized metric space}

\subsection{Basic definitions}

We start by outlining some basic elements of generalized metric spaces (for a general background see [34]).

Definition 3.1 A generalized metric space is a pair $(M, d)$, where $M$ is a set of points and $d: M \times M \rightarrow \mathbb{R}$ is a generalized distance (or generalized metric), i.e. $d$ satisfies the following axioms:

(a1) $d(x, y) \geq 0$ for all $x, y \in M$.

(a2) $d(x, y)=d(y, x)=0$ if and only if $x=y$.

(a3) $d(x, z) \leq d(x, y)+d(y, z)$ for all $x, y, z \in M$.

(a4) Given a sequence $\left\{x_{n}\right\} \subset M$ and $x \in M$, then $\lim _{n \rightarrow \infty} d\left(x_{n}, x\right)=0$ if and only if $\lim _{n \rightarrow \infty} d\left(x, x_{n}\right)=0$.

$A$ quasi-distance on $M$ is a map $d_{Q}: M \times M \rightarrow \mathbb{R} \cup\{\infty\}$ which satisfies the axioms (a1), (a2) and (a3) above.

The reversed generalized distance $d^{\mathrm{rev}}$ of a generalized metric $d$ is defined as $d^{\mathrm{rev}}(x, y)=$ $d(y, x)$, and the symmetrized distance $d^{s}$ as

$$
d^{s}(x, y)=\frac{1}{2}\left(d(x, y)+d^{\mathrm{rev}}(x, y)\right) .
$$

It is direct to check that $d^{s}$ is a true (symmetric) distance.

One can define for $d$ the notions of forward and backward balls of center $x \in M$ and radius $r \geq 0$; say, for open balls,

$$
B^{+}(x, r)=\{y \in M: d(x, y)<r\}, \quad B^{-}(x, r)=\{y \in M: d(y, x)<r\}, \quad \text { resp. }
$$

These balls coincide with the corresponding backward and forward balls for $d^{\text {rev }}$, resp. In what follows, we will use "forward elements", being the backward ones analogous (and corresponding to $d^{\text {rev }}$ ). The balls for the symmetrized distance will be denoted $B^{s}(x, r)$.

From the triangle inequality (a3), if $y \in B^{+}(x, r)$ one has $B^{+}\left(y, r^{\prime}\right) \subset B^{+}(x, r)$ whenever $0<r^{\prime}<r-d(x, y)$, and the forward open balls constitute a basis for a (first countable) topology. Moreover, with this topology a sequence $\left\{x_{n}\right\} \subset M$ converges to some $x \in M$ if and only if $d\left(x, x_{n}\right) \rightarrow 0$. By (a 4 ) this convergence is equivalent to the convergence with the topology generated by the open backward balls (which will be equal to the former forward topology).

Remark 3.2 Previous definitions are extended naturally to any quasi-distance $d_{Q}$. Recall that for such a $d_{Q}$ the hypothesis (a4) in Defn. 3.1 holds if and only if the topology generated by the forward balls coincide with the topology generated by the 
backward ones -and, then, also by the open symmetrized balls. So, any generalized metric or quasi-distance satisfying ( $a_{4}$ ) will be regarded as a topological space with the topology generated by the open forward and backward balls, and the limits in (a4) characterize the convergence of $\left\{x_{n}\right\}$ to $x$ in the unique natural sense.

Moreover, this condition (a 4 ) implies the following natural property: If $d_{Q}(x, y)=$ 0 then $d_{Q}(y, x)=0$, and thus, by (a2), $x=y$ (in particular, the topology is Hausdorff). So, the strengthening of (a2), $d(x, y)=0$ iff $x=y$, holds in any generalized metric space $(M, d)$, but it is easy to check that this property does not imply (a4) (see Example 3.24).

Remark 3.3 We will be interested in the study of Finsler manifolds $(M, F)$, which yield the generalized metric spaces $\left(M, d_{F}\right),\left(M, d_{F^{\mathrm{rev}}}\right)$ : in fact, these spaces satisfy condition (a 4 ) in Defn. 3.1 because the topologies associated to $d_{F}$ and $d_{F}^{\text {rev }}=d_{F \text { rev }}$ coincides with the topology of the manifold (see for example [3, Sect. 6.2 C]).

\subsection{Forward and backward Cauchy boundaries as point sets}

\subsubsection{Cauchy sequences and completions.}

Definition 3.4 A sequence $\sigma=\left\{x_{n}\right\}$ in a generalized metric space $(M, d)$ is a (forward) Cauchy sequence if for all $\epsilon>0$ there exists $n_{0}$ such that $d\left(x_{n}, x_{m}\right)<\epsilon$ whenever $n_{0} \leq n \leq m$. The space of all the Cauchy sequences will be denoted $\mathrm{Cau}^{+}(M)$ or, simply, Cau $(M)$. A backward Cauchy sequence is a (forward) Cauchy sequence for $d^{\text {rev }}$. The space of all the backward Cauchy sequences will be denoted by $\mathrm{Cau}^{-}(M)$.

Obviously, these definitions agree with the usual ones for (symmetric) distances, and are also extendible to quasi-distances. It is easy to check that any convergent sequence (with the natural topology, recall Remark 3.2 is Cauchy for a generalized metric (see Proposition 3.9 (3) below) but this property fails for a quasi-distance (Remark 3.26(4)).

Note the following straightforward assertion on double limits, which will be claimed several times:

Lemma 3.5 Two sequences $\sigma=\left\{x_{n}\right\}, \sigma^{\prime}=\left\{x_{n}^{\prime}\right\} \subset M$ satisfy $\lim _{n}\left(\lim _{m} d\left(x_{n}, x_{m}^{\prime}\right)\right)$ $=\ell, \ell \in \mathbb{R}$ (resp. $\ell=\infty$ ) if and only if for all $\epsilon>0$ there exists $n_{0}>0$ such that for all $n \geq n_{0}$ there is some $m_{0}(n)$ satisfying: if $m \geq m_{0}(n)$ then $\left|d\left(x_{n}, x_{m}^{\prime}\right)-\ell\right|<\epsilon$ (resp. $\left.d\left(x_{n}, x_{m}^{\prime}\right)>\epsilon\right)$.

As a first technical result we have:

Lemma 3.6 For any $x \in M$ and $\left\{x_{n}\right\},\left\{y_{n}\right\} \in \mathrm{Cau}(M)$, the sequence $\left\{d\left(x, y_{m}\right)\right\}$ converges in $\mathbb{R}$, the sequence $\left\{d\left(y_{m}, x\right)\right\}$ converges in $[0, \infty]$, and the double limit

$$
\ell=\lim _{n}\left(\lim _{m} d\left(x_{n}, y_{m}\right)\right)
$$

exists in $[0, \infty]$. Moreover, there exist subsequences $\left\{x_{n_{k}}\right\}_{k},\left\{y_{m_{k}}\right\}_{k}$ such that $\ell=$ $\lim _{k} d\left(x_{n_{k}}, y_{m_{k}}\right)$. 
Proof. For the first assertion (the second one is analogous), assume first that the sequence does not converge in $[0, \infty]$. Then, there exist two subsequences converging to different limits. Arranging appropriate subsequences $\left\{y_{m_{k}}\right\},\left\{y_{m_{k}^{\prime}}\right\}$ such that $m_{k}<m_{k}^{\prime}$ and

$$
0<\epsilon_{0} \leq d\left(x, y_{m_{k}^{\prime}}\right)-d\left(x, y_{m_{k}}\right) \leq d\left(y_{m_{k}}, y_{m_{k}^{\prime}}\right) \quad \forall k \in \mathbb{N},
$$

a contradiction with the fact that $\left\{y_{m}\right\}$ is a Cauchy sequence is obtained. For the finiteness of the limit, observe also that $\left\{d\left(x, y_{m}\right)\right\}_{m}$ is clearly bounded, and thus, it must converge in $\mathbb{R}$.

For the limit (3.2), denote $L_{n}=\lim _{m} d\left(x_{n}, y_{m}\right)$ and assume by contradiction that there exist subsequences $L_{n_{k}}, L_{n_{k}^{\prime}}$ such that

$$
0<2 \epsilon_{1}<L_{n_{k}^{\prime}}-L_{n_{k}}
$$

Without loss of generality, we can also assume $n_{k}^{\prime}<n_{k}$. Then, there exist two sequences $\left\{y_{m_{k}}\right\}$ and $\left\{y_{m_{k}^{\prime}}\right\}$ such that $m_{k}<m_{k}^{\prime}$ and

$$
0<\epsilon_{1} \leq d\left(x_{n_{k}^{\prime}}, y_{m_{k}^{\prime}}\right)-d\left(x_{n_{k}}, y_{m_{k}}\right) \leq d\left(x_{n_{k}^{\prime}}, x_{n_{k}}\right)+d\left(y_{m_{k}}, y_{m_{k}^{\prime}}\right) \quad \forall k \in \mathbb{N}
$$

in contradiction to the fact that $\left\{x_{n}\right\},\left\{y_{m}\right\}$ are both Cauchy sequences.

Finally, the last assertion follows easily from Lemma 3.5 .

Remark 3.7 As a technical question to be used later, notice that, if $\left\{x_{n}\right\} \in$ $\mathrm{Cau}(M)$ and $\left\{y_{n}\right\} \in \mathrm{Cau}^{-}(M)$, we still have that $\lim _{n}\left(\lim _{m} d\left(x_{n}, y_{m}\right)\right)$ exists in $[0, \infty]$. To check it, just interchange the roles of $y_{m_{k}}$ and $y_{m_{k}^{\prime}}$ in formula 3.3. Of course, by applying Lemma 3.6 to $d^{\text {rev }}$, we also have that $\left\{d\left(x, y_{m}\right)\right\}(=$ $\left.\left\{d^{\operatorname{rev}}\left(y_{m}, x\right)\right\}\right)$ converges in $[0, \infty]$ and $\left\{d\left(y_{m}, x\right)\right\}\left(=\left\{d^{\text {rev }}\left(x, y_{m}\right)\right\}\right)$ converges in $\mathbb{R}$.

Definition 3.8 Two Cauchy sequences $\sigma=\left\{x_{n}\right\}, \sigma^{\prime}=\left\{x_{n}^{\prime}\right\} \in \operatorname{Cau}(M)$ are related, $\sigma \sim \sigma^{\prime}$, if

$$
\lim _{n}\left(\lim _{m} d\left(x_{n}, x_{m}^{\prime}\right)\right)=\lim _{n}\left(\lim _{m} d\left(x_{n}^{\prime}, x_{m}\right)\right)=0 .
$$

Proposition 3.9 (1) The binary relation $\sim$ is a relation of equivalence on $\mathrm{Cau}(M)$.

(2) Let $x \in M, \sigma=\left\{x_{n}\right\}, \sigma^{\prime}=\left\{x_{n}^{\prime}\right\} \in \operatorname{Cau}(M)$. If $\sigma \sim \sigma^{\prime}$ then $\lim _{n} d\left(x_{n}, x\right)=$ $\lim _{n} d\left(x_{n}^{\prime}, x\right)(\in[0, \infty])$.

(3) If a representative of a class of equivalence converges, then any other representative of that class converges to the same limit. If two sequences $\left\{x_{n}\right\},\left\{x_{n}^{\prime}\right\}$ converge to the same point $x$, then both sequences are Cauchy and $\left\{x_{n}\right\} \sim\left\{x_{n}^{\prime}\right\}$.

Proof. (1) Symmetry and reflexivity are direct from the definitions. To prove transitivity, consider Cauchy sequences $\left\{x_{n}\right\} \sim\left\{x_{n}^{\prime}\right\} \sim\left\{x_{n}^{\prime \prime}\right\}$. Let $\epsilon>0$, by applying Lemma 3.5 to the first two sequences on the one hand, and to the last two sequences on the other hand, one obtains the corresponding natural numbers $n_{0}=$ : $n_{12}, m_{0}(n)=: m_{12}(n)$ and $n_{23}, m_{23}(n)$, resp. To check that $\left\{x_{n}\right\} \sim\left\{x_{n}^{\prime \prime}\right\}$, define 
$l(n):=\max \left\{m_{12}(n), n_{23}\right\}$ for each $n \geq n_{12}$. Then, use the claim with $n_{13}:=n_{12}$, $m_{13}(n):=m_{23}(l(n))$, and take into account that, for $n \geq n_{13}, m \geq m_{13}(n)$, it is

$$
d\left(x_{n}, x_{m}^{\prime \prime}\right) \leq d\left(x_{n}, x_{l(n)}^{\prime}\right)+d\left(x_{l(n)}^{\prime}, x_{m}^{\prime \prime}\right)<2 \epsilon .
$$

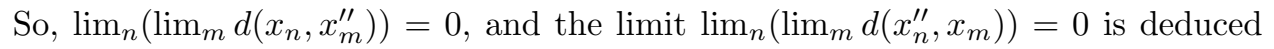
analogously.

(2) From Lemma 3.6 both limits exist in $[0, \infty]$, and

$$
\lim _{n} d\left(x_{n}, x\right) \leq \lim _{n}\left(\lim _{m}\left(d\left(x_{n}, x_{m}^{\prime}\right)+d\left(x_{m}^{\prime}, x\right)\right)\right)=\lim _{m} d\left(x_{m}^{\prime}, x\right) .
$$

So, $\lim _{n} d\left(x_{n}, x\right) \leq \lim _{m} d\left(x_{m}^{\prime}, x\right)$ and the converse is analogous.

(3) The first assertion is obvious from item (2) as the convergence is characterized by the limits with the distance (recall Remark 3.2, and its paragraph above)

For the last assertion, recall that $\lim _{n} d\left(x_{n}, x\right)=0=\lim _{n} d\left(x_{n}^{\prime}, x\right)=\lim _{n} d\left(x, x_{n}^{\prime}\right)$ (where the last equality follows from condition (a4) in Definition 3.1) and $d\left(x_{n}, x_{m}^{\prime}\right) \leq$ $d\left(x_{n}, x\right)+d\left(x, x_{m}^{\prime}\right)$. Then, for any $\epsilon>0$ there exists $n_{0}$ such that, for all $m, n \geq n_{0}$, $d\left(x_{n}, x_{m}^{\prime}\right)<\epsilon$. This last property ensures that $\left\{x_{n}\right\} \sim\left\{x_{n}^{\prime}\right\}$. For the Cauchy character of (each) sequence, put $x_{n}=x_{n}^{\prime}$ in previous proof (obviously, the sequence is then both, forward and backward Cauchy).

Remark 3.10 The first two assertions in Proposition 3.9 can be extended directly to Cauchy sequences of any set with a quasi-distance (for the third one, a discussion on the topologies associated to the quasi-distance is required, see Remark 3.26 below). This would allow to define also the Cauchy completion of any set endowed with a quasi-distance, as we will do next for generalized metric spaces (Defn. 3.11). Even though we will not be interested in this possibility, the extension of Proposition 3.9(2) to quasi-distances will be used below in order to ensure that the Cauchy completion of a generalized metric space becomes truly complete (see Theorem 3.29 (iii)).

Now, we are in conditions to define the (generalized) Cauchy completions.

Definition 3.11 The (forward) Cauchy completion $M_{C}^{+}$of a generalized metric space $(M, d)$ is the quotient space $M_{C}^{+}:=\operatorname{Cau}(M) / \sim$. Then, $M$ is regarded naturally as the subset of $M_{C}^{+}$consisting of the classes of equivalence of all the sequences converging in $M\left(\subset M_{C}^{+}\right)$. The (forward) Cauchy boundary of $(M, d)$ is $\partial_{C}^{+} M:=M_{C}^{+} \backslash M$.

The backward Cauchy completion $M_{C}^{-}$and boundary $\partial_{C}^{-} M$ are defined analogously by using the reversed generalized distance $d^{\text {rev }}$.

Remark 3.12 (a) In the Finslerian case, $d_{F}$ and $d_{F^{\text {rev }}}$ are generalized distances associated to a length space (in the sense of [16, 17]); this means that such distances are computable from the infimum of the (previously defined) lengths of suitable curves connecting two points. In particular, this implies that an appropriate version of Hopf-Rinow Theorem holds (see [8, Appendix] for further details). 
(b) Thus, the corresponding Cauchy boundaries can be constructed by using curves of finite length instead of Cauchy sequences. Namely, given a piecewise smooth curve (as in the case of spacetimes discussed in Remark 2.1, one can consider alternatively smooth or piecewise smoooth curves) $\gamma:[0, b) \rightarrow M, b<\infty$, parametrized with the length, any sequence $\left\{s_{n}\right\} \nearrow b$ yields a Cauchy sequence $\left\{\gamma\left(s_{n}\right)\right\}$-and all the so-obtained Cauchy sequences are equivalent for $\sim$. Conversely, given a Cauchy sequence, a curve $\gamma$ as above is obtained by connecting appropriate terms of the sequence by means of arc-parametrized segments with lengths approaching fast to the distances between their endpoints. This viewpoint will be used later, when the Cauchy completion is regarded as a part of the Busemann one.

\subsubsection{Alternative Cauchy sequences yield the same completions}

The equivalence relation defined by the double limits in (3.4) suggests the following alternative way to introduce the notion of Cauchy sequence.

Definition 3.13 A sequence $\sigma=\left\{x_{n}\right\}$ in a generalized metric space $(M, d)$ is a (forward) alternative Cauchy sequence if $\lim _{n}\left(\lim _{m} d\left(x_{n}, x_{m}\right)\right)=0$. The space of all the alternative Cauchy sequences will be denoted $\mathrm{Cau}_{\text {alt }}^{+}(M)$ or, simply, $\mathrm{Cau}_{\text {alt }}(M)$. $A$ backward alternative Cauchy sequence is a (forward) alternative Cauchy sequence for $d^{\text {rev }}$. The space of all the backward alternative Cauchy sequences will be denoted $\mathrm{Cau}_{\text {alt }}^{-}(M)$.

Let us summarize the elementary properties of alternative Cauchy sequences.

Proposition 3.14 Let $\sigma=\left\{x_{n}\right\}$ be a sequence in a generalized metric space $(M, d)$. Then:

(1) $\sigma$ is an alternative Cauchy sequence if and only if for all $\epsilon>0$ there exists $n_{0}$ such that for all $n \geq n_{0}$ there is $m_{0}(n)$ satisfying: if $m \geq m_{0}(n)$ then $d\left(x_{n}, x_{m}\right)<\epsilon$.

(2) Any Cauchy sequence is also an alternative Cauchy sequence.

(3) If $d$ is symmetric then the alternative Cauchy sequences coincide with the Cauchy ones.

Proof. (1) Apply Lemma 3.5 with $\sigma=\sigma^{\prime}$.

(2) From the definition of alternative Cauchy sequence, the property in (1) holds by putting $m_{0}(n)=n$.

(3) It reduces to prove the converse of (2). So, let $\sigma=\left\{x_{n}\right\}$ be an alternative Cauchy sequence, and $\epsilon>0$. Take $n_{0}$ and $m_{0}(n)$ as in (1), and define $m_{12}=\max \left\{m_{0}\left(n_{1}\right), m_{0}\left(n_{2}\right)\right\}$ for each $n_{1}, n_{2} \geq n_{0}$. Then:

$$
d\left(x_{n_{1}}, x_{n_{2}}\right) \leq d\left(x_{n_{1}}, x_{m_{12}}\right)+d\left(x_{m_{12}}, x_{n_{2}}\right)=d\left(x_{n_{1}}, x_{m_{12}}\right)+d\left(x_{n_{2}}, x_{m_{12}}\right)<2 \epsilon,
$$

as required. 
Example 3.15 Let us show that, in general, there are alternative Cauchy sequences which are not Cauchy sequences. Consider the Finsler manifold $(M, F)$ given by

$$
M=(0, \infty), \quad F=\sqrt{g_{0}+\omega^{2}}+\omega, \quad \text { where } g_{0}=\frac{d x^{2}}{1+x^{2}}, \quad \omega=-d x,
$$

and the sequence $\sigma=\left\{x_{n}\right\} \subset M, x_{n}=n+(-1)^{n+1}$. Observe that $d_{F}\left(x_{2 n-1}, x_{2 n}\right)>$ 2, and so, $\sigma$ is not a Cauchy sequence according to Defn. 3.4. However, $\sigma$ is an alternative Cauchy sequence according to Defn. 3.13 since

$$
\lim _{n}\left(\lim _{m} d_{F}\left(x_{n}, x_{m}\right)\right)=\lim _{n}\left(\lim _{m} \operatorname{length}_{F}\left(\left.\alpha\right|_{\left(x_{n}, x_{m}\right)}\right)\right)=0, \quad \text { where } \alpha(t)=t .
$$

The relevance of the notion of alternative Cauchy sequence for the Cauchy completion is stressed in the first assertion of the following proposition.

Proposition 3.16 (1) Let $\sigma, \sigma^{\prime}$ be two arbitrary sequences in a generalized metric space $(M, d)$. If the double limits (3.4) hold then $\sigma$ and $\sigma^{\prime}$ are alternative Cauchy sequences.

(2) The extension of the binary relation $\sim\left(\right.$ Defn. 3.8) to the set $\mathrm{Cau}_{\mathrm{alt}}(M)$ of all the alternative Cauchy sequences is also a relation of equivalence.

(3) If a representative of a class of equivalence in $\mathrm{Cau}_{\text {alt }}(M) / \sim$ converges, then all the representatives of that class converge to the same limit.

Proof. We will prove that $\sigma^{\prime}$ is an alternative Cauchy sequence by using Prop. 3.14 (1), being the proof of the remainder assertions similar to the case of Cauchy sequences. Given $\epsilon>0$, use the values of $n_{1}$ and $m_{1}(n)$ (for $n \geq n_{1}$ ) in Lemma 3.5 so that $d\left(x_{n}^{\prime}, x_{m}\right)<\epsilon$ whenever $m \geq m_{1}(n)$. Analogously, let $n_{2}>0$ and $m_{2}(n)$ (for $n \geq n_{2}$ ) so that $d\left(x_{n}, x_{m}^{\prime}\right)<\epsilon$ whenever $m \geq m_{2}(n)$. Put $n_{0}:=n_{1}$ and, for $n \geq n_{0}, k(n):=\max \left\{m_{1}(n), n_{2}\right\}$ and $m_{0}(n):=m_{2}(k(n))$. Then, if $n \geq n_{0}$ and $m \geq m_{0}(n)$, it is

$$
d\left(x_{n}^{\prime}, x_{m}^{\prime}\right) \leq d\left(x_{n}^{\prime}, x_{k(n)}\right)+d\left(x_{k(n)}, x_{m}^{\prime}\right)<2 \epsilon,
$$

as required.

Theorem 3.17 Any alternative Cauchy sequence $\sigma$ contains some subsequence $\sigma^{\prime}$ which is a Cauchy sequence, and $\sigma \sim \sigma^{\prime}$ (i.e. (3.4) holds). Moreover, $\sigma$ converges if and only if so does $\sigma^{\prime}$, and the natural projection

$$
\left(\mathrm{Cau}_{\mathrm{alt}}(M) / \sim\right) \rightarrow(\mathrm{Cau}(M) / \sim), \quad[\sigma] \mapsto\left[\sigma^{\prime}\right]
$$

is well-defined and bijective.

Proof. Let $\sigma=\left\{x_{n}\right\}$, and define inductively the subsequence $\sigma^{\prime}$ as follows. For $k=0$, put $n_{0}=m\left(n_{0}\right)=1$ and, for each integer $k \geq 1$ take $\epsilon=1 / k$. From Lemma 3.5 there exists $n_{k} \geq m\left(n_{k-1}\right)$ and $m\left(n_{k}\right) \geq n_{k}$ such that $d\left(x_{n_{k}}, x_{m}\right)<1 / k$ for all $m \geq m\left(n_{k}\right)$. Then, the subsequence $\left\{x_{n_{k}}\right\}_{k} \subset\left\{x_{n}\right\}_{n}$ is a Cauchy sequence according to Defn. 3.4. In fact, for every $k$, if $k_{2} \geq k_{1} \geq k$ then $n_{k_{1}} \geq n_{k}$ and $n_{k_{2}} \geq m\left(n_{k_{2}-1}\right) \geq m\left(n_{k_{1}}\right)$, and so $d\left(x_{n_{k_{1}}}, x_{n_{k_{2}}}\right)<1 / k$. The remainder is straightforward. 
Remark 3.18 Theorem 3.17 ensures that, if we define the (forward) Cauchy completion by using alternative Cauchy sequences instead of Cauchy sequences, the resulting space remains the same. This shows the consistency of our approach against possible alternatives.

Notice that the consistency also includes the convergence of sequences. In particular, the Finslerian version of Hopf-Rinow Theorem also holds if, instead of considering Cauchy sequences, one uses alternative Cauchy sequences.

In what follows, we will use Cauchy sequences instead of alternative ones, as the first set is smaller $\left(\mathrm{Cau}(M) \subset \mathrm{Cau}^{\text {alt }}(M)\right)$ and more classical.

The next result summarizes the natural consistency between the notion of Cauchy sequence and the relation of equivalence $\sim$. It is straightforward from Prop. 3.16 (1) and Theorem 3.17 .

Corollary 3.19 Let $\sigma \in \operatorname{Cau}(M)$ and $\sigma^{\prime}$ any sequence in $M$. If the double limits (3.4) hold, then $\sigma^{\prime}$ contains some subsequence $\sigma^{\prime \prime} \in \mathrm{Cau}(M)$ satisfying $\sigma \sim \sigma^{\prime \prime}$. In this case, $\sigma$ is convergent if and only if so is $\sigma^{\prime \prime}$, and the limits of both sequences coincide.

\subsubsection{The symmetrized Cauchy boundary}

Even though the forward and backward boundaries have been defined in terms of different objects, the following technical result allows to establish a precise relation between them. We always consider $\sigma=\left\{x_{n}\right\}, \sigma^{\prime}=\left\{x_{n}^{\prime}\right\}$.

Proposition 3.20 If $\sigma, \sigma^{\prime} \in \mathrm{Cau}(M)$ are equivalent for $d$ and $\sigma \in \mathrm{Cau}^{-}(M)$, then $\sigma^{\prime} \in \mathrm{Cau}^{-}(M)$ and $\sigma, \sigma^{\prime}$ are also equivalent for $d^{\mathrm{rev}}$.

Proof. To prove $\sigma^{\prime} \in \mathrm{Cau}^{-}(M)$, take $\epsilon>0$ and let $n_{1}, m_{1}(n), n_{3}, m_{3}(n)$ be the natural numbers obtained by applying Lemma 3.5 to the equalities

$$
\lim _{n}\left(\lim _{m} d\left(x_{n}, x_{m}^{\prime}\right)\right)=0, \quad \lim _{n}\left(\lim _{m} d\left(x_{n}^{\prime}, x_{m}\right)\right)=0 \quad\left(\text { recall that } \sigma \sim \sigma^{\prime} \text { for } d\right) .
$$

Since $\sigma \in \mathrm{Cau}^{-}(M)$, there exists $n_{2}$ such that if $m \geq n \geq n_{2}$ then

$$
d\left(x_{m}, x_{n}\right)<\epsilon .
$$

Let $n_{0}:=\max \left\{n_{1}, n_{2}\right\}, N_{0}:=\max \left\{n_{3}, m_{1}\left(n_{0}\right)\right\}$. For any $m \geq n \geq N_{0}$, take some $k \geq \max \left\{m_{3}(m), n_{0}\right\}$. Then,

$$
d\left(x_{m}^{\prime}, x_{n}^{\prime}\right) \leq d\left(x_{m}^{\prime}, x_{k}\right)+d\left(x_{k}, x_{n_{0}}\right)+d\left(x_{n_{0}}, x_{n}^{\prime}\right)<3 \epsilon,
$$

and $\sigma^{\prime} \in \mathrm{Cau}^{-}(M)$. To prove that $\sigma, \sigma^{\prime}$ are related for $d^{\text {rev }}$, consider the natural numbers $n_{1}, m_{1}(n), n_{2}, n_{0}=\max \left\{n_{1}, n_{2}\right\}$ obtained as above, and let $N_{0}^{\prime}:=$ $\max \left\{n_{0}, m_{1}\left(n_{0}\right)\right\}$. If $m \geq n \geq N_{0}^{\prime}$ then

$$
d\left(x_{m}, x_{n}^{\prime}\right) \leq d\left(x_{m}, x_{n_{0}}\right)+d\left(x_{n_{0}}, x_{n}^{\prime}\right)<2 \epsilon .
$$


Therefore, $\lim _{n}\left(\lim _{m} d^{\mathrm{rev}}\left(x_{n}^{\prime}, x_{m}\right)\right)=0$. The other limit follows by interchanging the roles of both sequences.

With this result in mind, one can identify points in $\partial_{C}^{+} M$ with points in $\partial_{C}^{-} M$ by requiring that they are represented by the same class of Cauchy sequences. In particular, the following natural definition makes sense:

Definition 3.21 Let $(M, d)$ be a generalized metric space. The symmetrized Cauchy boundary of $M$ is the intersection of its two Cauchy boundaries:

$$
\left.\partial_{C}^{s} M:=\partial_{C}^{+} M \cap \partial_{C}^{-} M \quad \text { (and then, } M_{C}^{s}:=M \cup \partial_{C}^{s} M\right) .
$$

Easily, $\partial_{C}^{s} M$ can be also computed in terms of the symmetrized distance in (3.1):

Proposition 3.22 A sequence $\sigma$ is Cauchy for $d^{s}$ if and only if it belongs to $\partial_{C}^{s} M$. Thus, the symmetrized Cauchy boundary $\partial_{C}^{s} M$ is equal to the Cauchy boundary for the symmetrized distance $d^{s}$.

Remark 3.23 For a Riemannian metric, one has a unique Cauchy boundary, namely $\partial_{C}^{s} M$, which is associated to a length space (see Remark 3.12). Nevertheless, in the Finslerian case, the symmetrized distance $d^{s}$ is not associated to a length space (see [8. Appendix] and [8. Example 2.3] for details). So, the construction of $\partial_{C}^{s} M$ cannot be carried out by means of lengths of curves in the natural way. This prevents us to consider the symmetrized Cauchy boundary $\partial_{C}^{s} M$ as a sort of Riemannian boundary in general.

The next simple example justifies some of the cautions adopted with double limits along this section, and motivates the next one.

Example 3.24 In this Finslerian example we show two Cauchy sequences in $\partial_{C}^{s} M$ satisfying that only one of the double limit equalities in 3.4 holds. Let $D_{*} \subset \mathbb{R}^{2}$ be the open disk of radius 1 where the segment $[-1,0]$ of the horizontal axis has been removed. Define $F=\sqrt{d r^{2}+r^{2} d \theta^{2}+(1-r)^{2} d \theta^{2}}-(1-r) d \theta$ in polar coordinates $r, \theta$. Consider the sequences $\left\{x_{n}=(r=1 / n, \theta=0)\right\}_{n},\left\{x_{n}^{\prime}=\left(r=1 / n, \theta=\frac{\pi}{2}\right)\right\}_{n}$, whose classes of equivalence clearly belong to $\partial_{C}^{s} M$. Note that:

$$
\lim _{n}\left(\lim _{m} d_{F}\left(x_{n}, x_{m}^{\prime}\right)\right)=0, \quad \lim _{n}\left(\lim _{m} d_{F}\left(x_{n}^{\prime}, x_{m}\right)\right) \geq \pi / 4 .
$$

In fact, for the first limit consider arc curves (of constant radius) from $x_{n}$ to $x_{n}^{\prime}$, and then, radial curves from $x_{n}^{\prime}$ to $x_{m}^{\prime}$. For the second one, consider any curve $\gamma:[a, b] \rightarrow D_{*}, \gamma(s)=(r(s), \theta(s))$ joining $x_{n}^{\prime}$ and $x_{m}$, and let $J:=\{s \in[a, b]:$ $\dot{\theta}(s) \leq 0\}$. Then, $\left[0, \frac{\pi}{2}\right] \subset \theta(J)$, and so,

$\operatorname{length}_{F}(\gamma) \geq \int_{J} F(\dot{\gamma})=\int_{J}\left(\sqrt{\dot{r}^{2}+r^{2} \dot{\theta}^{2}+(1-r)^{2} \dot{\theta}^{2}}-(1-r) \dot{\theta}\right)>\frac{1}{2} \int_{J}|\dot{\theta}| \geq \frac{\pi}{4}$. 


\subsection{Quasi-distance and topology on the Cauchy completions}

Example 3.24 also shows that, if a generalized distance $d$ is extended naturally to $M_{C}^{+}$, then such an extension may not be a generalized distance. However, it will be still a quasi-distance (Defn. 3.1):

Proposition 3.25 The map $d_{Q}: M_{C}^{+} \times M_{C}^{+} \rightarrow[0, \infty]$ given by

$$
d_{Q}\left(\left[\left\{x_{n}\right\}\right],\left[\left\{y_{n}\right\}\right]\right):=\lim _{n}\left(\lim _{m} d\left(x_{n}, y_{m}\right)\right)
$$

is well-defined and becomes a quasi-distance on $M_{C}^{+}$.

Analogously, $d^{\mathrm{rev}}$ extends to a quasi-distance $d_{Q}^{\mathrm{rev}}$ on $M_{C}^{-}$, and $d^{s}$ extends to a distance $\bar{d}^{s}: M_{C}^{s} \times M_{C}^{s} \rightarrow \mathbb{R}$, which satisfies $\bar{d}^{s}=\left(d_{Q}+d_{Q}^{\text {rev }}\right) / 2$ on $M_{C}^{s}$.

Proof. For the assertions on $d_{Q}$ (the assertions on $d_{Q}^{\text {rev }}$ are proved analogously), note that, from Lemma 3.6 , the limits type $\lim _{n}\left(\lim _{m} d\left(x_{n}, y_{m}\right)\right)$ always exist. In particular, they still exist if the sequences $\left\{x_{n}\right\},\left\{y_{n}\right\}$ are replaced by subsequences $\left\{x_{n_{k}}\right\}_{k},\left\{y_{n_{k}}\right\}_{k}$, and $\lim _{n}\left(\lim _{m} d\left(x_{n}, y_{m}\right)\right)=\lim _{k} d\left(x_{n_{k}}, y_{m_{k}}\right)$.

If $\left\{x_{n}\right\} \sim\left\{x_{n}^{\prime}\right\}$ and $\left\{y_{m}\right\} \sim\left\{y_{m}^{\prime}\right\}$, take subsequences $\left\{x_{n_{k}}\right\}_{k},\left\{x_{n_{k}^{\prime}}^{\prime}\right\}_{k}$ and $\left\{y_{m_{l}}\right\}_{l}$, $\left\{y_{m_{l}^{\prime}}^{\prime}\right\}_{l}$ such that $\lim _{k} d\left(x_{n_{k}}, x_{n_{k}^{\prime}}^{\prime}\right)=0, \lim _{l} d\left(y_{m_{l}^{\prime}}^{\prime}, y_{m_{l}}\right)=0$. Then,

$$
\begin{aligned}
\lim _{n}\left(\lim _{m} d\left(x_{n}, y_{m}\right)\right) & =\lim _{k}\left(\lim _{l} d\left(x_{n_{k}}, y_{m_{l}}\right)\right) \\
& \leq \lim _{k}\left(\lim _{l}\left(d\left(x_{n_{k}}, x_{n_{k}^{\prime}}^{\prime}\right)+d\left(x_{n_{k}^{\prime}}^{\prime}, y_{m_{l}^{\prime}}^{\prime}\right)+d\left(y_{m_{l}^{\prime}}^{\prime}, y_{m_{l}}\right)\right)\right) \\
& =\lim _{k}\left(\lim _{l} d\left(x_{n_{k}^{\prime}}^{\prime}, y_{m_{l}^{\prime}}^{\prime}\right)\right) \\
& =\lim _{n}\left(\lim _{m} d\left(x_{n}^{\prime}, y_{m}^{\prime}\right)\right),
\end{aligned}
$$

and analogously for the reversed inequality. Therefore, $d_{Q}$ is well-defined.

In order to prove that $d_{Q}$ is a quasi-distance on $M_{C}^{+}$, first observe that the equivalence $d_{Q}(x, y)=d_{Q}(y, x)=0$ iff $x=y$ becomes obvious; in fact, the former holds iff $\left\{x_{n}\right\} \sim\left\{y_{n}\right\}$, for $x \equiv\left[\left\{x_{n}\right\}\right], y \equiv\left[\left\{y_{n}\right\}\right]$, i.e. iff $x=y$. For the triangle inequality, consider $x \equiv\left[\left\{x_{n}\right\}\right], y \equiv\left[\left\{y_{m}\right\}\right], z \equiv\left[\left\{z_{l}\right\}\right]$ and take subsequences $\left\{y_{m_{n}}\right\}_{n},\left\{z_{l_{n}}\right\}_{n}$ such that

$$
d_{Q}(x, y)=\lim _{n} d\left(x_{n}, y_{m_{n}}\right), \quad d_{Q}(x, z)=\lim _{n} d\left(x_{n}, z_{l_{n}}\right), \quad d_{Q}(y, z)=\lim _{n} d\left(y_{m_{n}}, z_{l_{n}}\right)
$$

(for the last one, take a subsequence of the previous $l_{n}$ and rename it). Then,

$$
d_{Q}(x, z)=\lim _{n} d\left(x_{n}, z_{l_{n}}\right) \leq \lim _{n}\left(d\left(x_{n}, y_{m_{n}}\right)+d\left(y_{m_{n}}, z_{l_{n}}\right)\right)=d_{Q}(x, y)+d_{Q}(y, z) .
$$

Finally, the assertions on $d^{s}$ follow easily from Prop. 3.22 , and the usual properties of distances.

Remark 3.26 (1) As $d_{Q}$ is only a quasi-distance, there are two natural topologies on $M_{C}^{+}$: the one generated by the forward open balls of $d_{Q}$, and the one generated by the backward open balls. If $\left\{z_{n}\right\} \subset M_{C}^{+}$then:

$$
\begin{array}{llll}
z_{n} \rightarrow z \in M_{C}^{+} & \text {(forward balls topology) } & \Leftrightarrow & d_{Q}\left(z, z_{n}\right) \rightarrow 0 \\
z_{n} \rightarrow z \in M_{C}^{+} & \text {(backward balls topology) } & \Leftrightarrow & d_{Q}\left(z_{n}, z\right) \rightarrow 0 .
\end{array}
$$


These two topologies are different in general. In fact, Example 3.24 shows two points $z=\left[\left\{x_{n}\right\}\right], z^{\prime}=\left[\left\{x_{n}^{\prime}\right\}\right] \in \partial_{C}^{s} M \subseteq \partial_{C}^{+} M$ such that $d_{Q}\left(z, z^{\prime}\right)=0$ and $d_{Q}\left(z^{\prime}, z\right)>0$. So, any forward ball containing $z$ also contains $z^{\prime}$ (and any backward ball containing $z^{\prime}$ also contains $z$ ) but the converse does not hold. In particular, the topology in $M_{C}^{+}$generated by the backward balls is not $T_{1}$ - even though it is necessarily $T_{0}$. Note also that $\bar{d}^{s}$ becomes a true distance (Prop. 3.25, in spite of the fact that $z, z^{\prime} \in \partial_{C}^{s} M$.

(2) The character of quasi-distance for $d_{Q}$ is sharpened in Fig. 1 In this figure, the Randers manifold $(M, F)$ admits two boundary points $z_{1}, z_{2} \in \partial_{C}^{+} M$ such that $d_{Q}\left(z_{1}, z_{2}\right)=0$ and $d_{Q}\left(z_{2}, z_{1}\right)=\infty$. So, again the forward and backward topologies on $M_{C}^{+}$are different and non- $T_{1}$. Note also that $\bar{d}^{s}$ remains finite, as $M_{C}^{s}=M$.

(3) Even though the names suggest that the topology generated by the forward open balls is the natural one in $M_{C}^{+}$, this is not the case, because of the following completeness property: with the topology generated by the backward open balls, every (forward) Cauchy sequence $\left\{x_{n}\right\} \subset M$ converges to the point $z=\left[\left\{x_{n}\right\}\right]$ which defines in $M_{C}^{+}$. In fact,

$$
\lim _{n} d\left(x_{n}, z\right)=\lim _{n}\left(\lim _{m} d\left(x_{n}, x_{m}\right)\right)=0
$$

in agreement with the second equivalence in (3.6). In general, this property does not hold for the topology generated by the forward balls (see Examples 3.15, 3.24).

(4) As a relevant difference with the case of generalized distances, a convergent sequence for a quasi-distance (with the above natural topology) may not be Cauchy. In fact, consider again in Figure 1 the sequence $\left\{y_{n}\right\}$ given by $y_{2 n+1}=c_{1}(n)$ (the starting point of $\gamma_{n}$ ) and $y_{2 n}=c_{2}(n)$ (the endpoint). As $c_{1}$ defines $z_{1}, c_{2}$ defines $z_{2}$ and $d\left(z_{1}, z_{2}\right)=0$, the sequence $\left\{y_{n}\right\}$ satisfies $\lim _{n} d\left(y_{n}, z_{2}\right)=0$, even though $\left\{y_{n}\right\}$ is not Cauchy, as $d\left(y_{2 n}, y_{2 n+1}\right)$ does not converge to 0 .

Convention 3.27 The (forward) Cauchy completion $M_{C}^{+}$will be regarded as a topological space with the topology generated by the backward balls for the quasidistance $d_{Q}$ in 3.5 . So, the convergence of a sequence in $M_{C}^{+}$is characterized by the second relation in 3.6 , and each $\left\{x_{n}\right\} \in \operatorname{Cau}(M)$ converges to $\left[\left\{x_{n}\right\}\right] \in M_{C}^{+}$.

At any case, if $d_{Q}$ is a generalized distance, everything is simplified:

Proposition 3.28 If $d_{Q}$ is a generalized distance, then $\partial_{C}^{ \pm} M=\partial_{C}^{s} M$.

Proof. Consider $z=\left[\left\{x_{n}\right\}\right] \in \partial_{C}^{+} M$ and observe that $\lim _{n} d_{Q}\left(x_{n}, z\right)=0$ (recall Remark $3.26(3))$. From the generalized character of $d_{Q}, \lim _{n} d_{Q}\left(z, x_{n}\right)=0$ and, from definition of $d_{Q}, \lim _{n} \lim _{m} d\left(x_{m}, x_{n}\right)=0$, i.e. the sequence $\left\{x_{n}\right\}$ is an alternative backward Cauchy sequence. Finally, from Theorem 3.17 there exists a subsequence $\left\{x_{n_{k}}\right\}$ which is a backward Cauchy sequence, i.e. $z=\left[\left\{x_{n_{k}}\right\}\right] \in \partial_{C}^{-} M \cap \partial_{C}^{+} M=$ $\partial_{C}^{s} M$.

Theorem 3.29 The (forward) Cauchy completion $M_{C}^{+}$of a generalized metric space $(M, d)$ endowed with its natural (backward) topology in Convention 3.27 satisfies: 


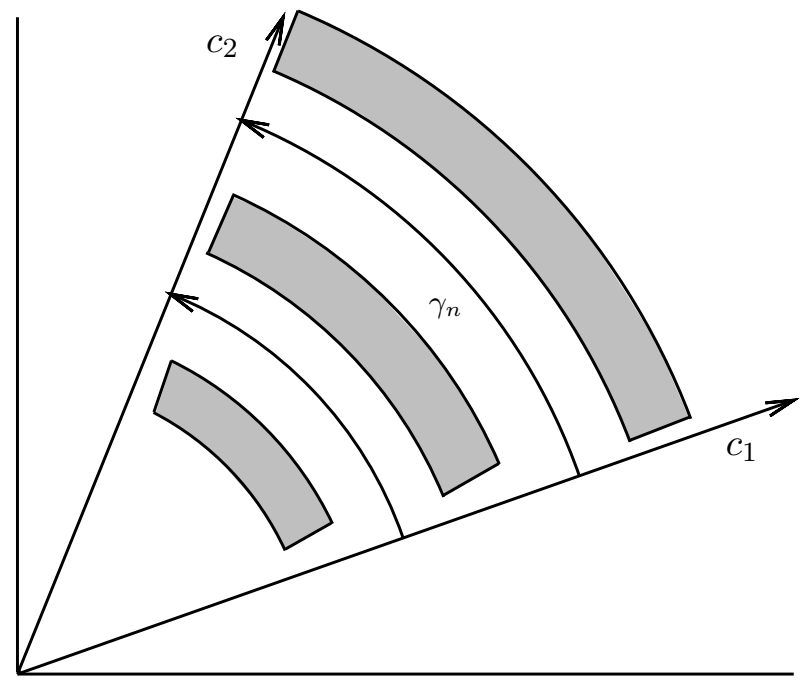

Figure 1: Limit situation for the quasi-distance $d_{Q}$ (see Remark 3.26).

An open subset $M$ of $\mathbb{R}^{2}$ (the region in grey is removed) with a Randers metric $F=$ $\sqrt{d x^{2}+d y^{2}+\omega^{2}}+\omega$. The radial dependence assumed for $\omega$ makes the rays $c_{1}, c_{2}$ to have finite length in the direction of the arrows; so, they determine some $z_{1}, z_{2} \in$ $\partial_{C}^{+} M$. The length is infinity in the opposite direction. The angular dependence assumed for $\omega$ makes the lengths of curves $\gamma_{n}$ to approach to 0 in the direction of the arrows and to infinite in the opposite direction. So, $d_{Q}\left(z_{1}, z_{2}\right)=0, d_{Q}\left(z_{2}, z_{1}\right)=\infty$. 
(i) The topology is $T_{0}$, and if two distinct points of $M_{C}^{+}$are not Hausdorff related then they both lie at the boundary $\partial_{C}^{+} M$.

(ii) The inclusion $i: M \rightarrow M_{C}^{+}$is a homeomorphism onto its image $i(M)$, and $i(M)$ is dense in $M_{C}^{+}$.

(iii) The quasi-distance $d_{Q}$ is forward complete, i.e. any forward Cauchy sequence $\left\{z_{n}\right\} \subset M_{C}^{+}$is convergent in $M_{C}^{+}$.

The analogous statements hold for $d_{Q}^{\mathrm{rev}}$ on $M_{C}^{-}$, while $\left(M_{C}^{s}, \bar{d}^{s}\right)$ is a (necessarily $\left.T_{2}\right)$ metric space.

Proof. (i) If $x, y \in M_{C}^{+}$are not $T_{0}$-separated, then $y \in B^{-}\left(x, \frac{1}{n}\right)$ and $x \in B^{-}\left(y, \frac{1}{n}\right)$ for all $n$. Thus, $d_{Q}(x, y)=d_{Q}(y, x)=0$ and $x=y$, as $d_{Q}$ is a quasi-distance.

Now, let $x, y \in M_{C}^{+}$be non-Hausdorff related, and assume $x \in M$. Taking into account that $B^{-}\left(x, \frac{1}{n}\right) \cap B^{-}\left(y, \frac{1}{n}\right) \neq \emptyset$ for all $n \in \mathbb{N}$, consider a sequence $\left\{x_{n}\right\} \subset M$ with $x_{n} \in B^{-}\left(x, \frac{1}{n}\right) \cap B^{-}\left(y, \frac{1}{n}\right)$. Then, $\lim _{n} d_{Q}\left(x_{n}, x\right)=0$ and, as $d_{Q}$ agrees with the generalized distance $d$ on $M, \lim _{n} d_{Q}\left(x, x_{n}\right)=0$. Therefore

$$
0 \leq d_{Q}(x, y) \leq d_{Q}\left(x, x_{n}\right)+d_{Q}\left(x_{n}, y\right) \rightarrow 0 \quad \text { as } n \rightarrow \infty .
$$

Therefore, if $y=\left[\left\{y_{n}\right\}\right]$, necessarily $0=d_{Q}(x, y)=\lim _{n} d_{Q}\left(x, y_{n}\right)$, i.e. $\left\{y_{n}\right\}$ converges to $x$ with the topology generated by the forward balls. As this topology coincides on $M$ with the one generated by the backward balls, one also have $\lim _{n} d_{Q}\left(y_{n}, x\right)=0$, i.e. $x$ is identifiable to $\left[\left\{y_{n}\right\}\right]=y$.

(ii) Straightforward.

(iii) Let $\left\{z_{n}\right\}_{n} \subset M_{C}^{+}, z_{n}=\left[\left\{z_{n}^{i}\right\}_{i}\right]$ be a Cauchy sequence. There is no loss of generality if we suppose that $d_{Q}\left(z_{n}, z_{n+1}\right)<\frac{1}{n^{2}}$ for all $n$. In fact, otherwise some subsequence $\left\{z_{n_{k}}\right\}_{k} \sim\left\{z_{n}\right\}_{n}$ would satisfy this property, and Proposition 3.9 (2) would be applicable (recall Remark 3.10). Consider the sequence $\left\{x_{n}\right\}$ defined as follows. For each $n$, Lemma 3.6 implies that, up to subsequences, there exist $i_{n}, j_{n}$ such that, for $i \geq i_{n}, j \geq j_{n}, d\left(z_{n}^{i}, z_{n+1}^{j}\right)<1 / n^{2}$. Then, choose $x_{1}=z_{1}^{i_{1}}$ and $x_{n}=z_{n}^{k(n)}$ where $k(n)=\operatorname{Max}\left\{i_{n}, j_{n-1}\right\}$.

One has to prove that $\left\{x_{n}\right\}$ is a Cauchy sequence, and the point $x=\left[\left\{x_{n}\right\}\right] \in M_{C}^{+}$ satisfies $\lim _{n} d_{Q}\left(z_{n}, x\right)=0$. For the first property, just notice that $d\left(x_{n}, x_{n+1}\right)=$ $d\left(z_{n}^{k(n)}, z_{n+1}^{k(n+1)}\right)<\frac{1}{n^{2}}$, and, so, $d\left(x_{n}, x_{n+h_{0}}\right)<\sum_{h=0}^{\infty}(n+h)^{-2}$. The second one follows from the following estimation:

$$
\begin{aligned}
d_{Q}\left(z_{n}, x\right) & =\lim _{i}\left(\lim _{m} d\left(z_{n}^{i}, x_{m}\right)\right) \\
& \leq \lim _{i}\left(\lim _{m}\left(d\left(z_{n}^{i}, x_{n+1}\right)+\sum_{j=n+1}^{m-1} d\left(x_{j}, x_{j+1}\right)\right)\right) \\
& =\lim _{i}\left(d\left(z_{n}^{i}, z_{n+1}^{k(n+1)}\right)\right)+\lim _{m}\left(\sum_{j=n+1}^{m-1} d\left(x_{j}, x_{j+1}\right)\right) . \\
& <\frac{1}{n^{2}}+\lim _{m} \sum_{j=n+1}^{m-1} \frac{1}{j^{2}} \\
& =\sum_{j=n}^{\infty} \frac{1}{j^{2}}
\end{aligned}
$$

Finally, for the last assertion recall Prop. 3.25

For Riemannian manifolds the Cauchy boundary is a closed subset of the Cauchy completion. Of course, this property cannot be expected for an arbitrary metric space $(X, d)$ (as its Cauchy completion is also the Cauchy completion of any dense, non necessarily open, subset $A \subset X)$. But such a property can be extended to the 
Finsler case (as well as more general spaces as those under Conventions 4.1, 5.1 below, as local compactness will be the essential property needed for the proof).

Proposition 3.30 For any Finsler manifold $(M, F)$, the boundary $\partial_{C}^{+} M$ is a closed subset of $M_{C}^{+}$.

Proof. Notice that, for any $x \in M$, any closed ball centered at $x$ with small radius $r>0$ is a compact subset of $M$ and, so, cannot contain a non-converging Cauchy sequence (nor a point of $\partial_{C}^{+} M$ ).

\subsection{Relating $M_{C}^{+}$and $M_{C}^{-}$through the extended quasi-distance}

Our aim here is to show that the extended quasi-distance $d_{Q}$ can be extended further to a domain larger than $M_{C}^{+}$.

Convention 3.31 From now on, and whenever there is no possibility of confusion, we will drop the notational distinction between $d, d_{Q}$ (resp. $\left.d^{\text {rev }}, d_{Q}^{\text {rev }}\right)$ and the further extensions of the generalized distance to be defined in the next proposition. So, $d$ and $d^{\text {rev }}$ will be applied in each case to the biggest possible domain.

Proposition 3.32 Let $(M, d)$ be a generalized metric space. Then:

(i) The maps $d: M_{C}^{+} \times\left(M_{C}^{+} \cup M_{C}^{-}\right) \rightarrow[0, \infty]$ and $d^{\mathrm{rev}}: M_{C}^{-} \times\left(M_{C}^{+} \cup M_{C}^{-}\right) \rightarrow$ $[0, \infty]$, given formally by the double limit 3.5), are well-defined.

(ii) $d(x, y)=d^{\mathrm{rev}}(y, x)$ for all $x \in M_{C}^{+}, y \in M_{C}^{-}$.

Proof. Let $x=\left[\left\{x_{n}\right\}\right]=\left[\left\{x_{n}^{\prime}\right\}\right] \in M_{C}^{+}, y=\left[\left\{y_{n}\right\}\right]=\left[\left\{y_{n}^{\prime}\right\}\right] \in M_{C}^{-}$.

(i) From Remark 3.7, the double $\operatorname{limits}_{\lim }\left(\lim _{m} d\left(x_{n}, y_{m}\right)\right)$ and $\lim _{n}\left(\lim _{m} d\left(x_{n}^{\prime}, y_{m}^{\prime}\right)\right)$ exist in $[0, \infty)$. Then, the same proof in Prop. 3.25 shows that they coincide.

(ii) Clearly,

$$
\begin{aligned}
d^{\mathrm{rev}}(y, x) & =\lim _{n}\left(\lim _{m} d^{\mathrm{rev}}\left(y_{n}, x_{m}\right)\right)=\lim _{n}\left(\lim _{m} d\left(x_{m}, y_{n}\right)\right) \\
& \leq \lim _{n}\left(\lim _{m}\left(\lim _{k}\left(d\left(x_{m}, y_{k}\right)+d\left(y_{k}, y_{n}\right)\right)\right)\right) \\
& =\lim _{m}\left(\lim _{k} d\left(x_{m}, y_{k}\right)\right)+\lim _{n}\left(\lim _{k} d^{\mathrm{rev}}\left(y_{n}, y_{k}\right)\right) \\
& =d(x, y)+d^{\mathrm{rev}}(y, y)=d(x, y)
\end{aligned}
$$

and the reversed inequality holds by interchanging the roles of $x$ and $y$.

Remark 3.33 In the previous proposition, the value $\infty$ is allowed for $d$. Only when $x \in M$ and $x^{+} \in M_{C}^{+}$, we can ensure $d\left(x, x^{+}\right)<\infty$ (Lemma 3.6), and analogously for $d^{\text {rev }}$.

In the case of length spaces, one can connect any $x \in M$ with $x^{+}=\left[\left\{x_{n}\right\}\right] \in$ $\partial_{C}^{+} M$ by means of a curve of finite length: in fact, for certain subsequence $\left\{n_{i}\right\}$, one can join $x$ with $x_{n_{1}}$ by a curve of finite length, and each $x_{n_{i}}$ with $x_{n_{i+1}}$ by a curve of length smaller than $i^{-2}$. If the reversed curve of $\gamma$ had also finite length, then one would also have $d\left(x^{+}, x\right)<\infty$ and $x^{+} \in \partial_{C}^{s} M$. However, even if we know that $d\left(x^{+}, x\right)<\infty$, we cannot ensure that $x^{+}$belongs to $\partial_{C}^{s} M$. The reason is that $x^{+}$ 


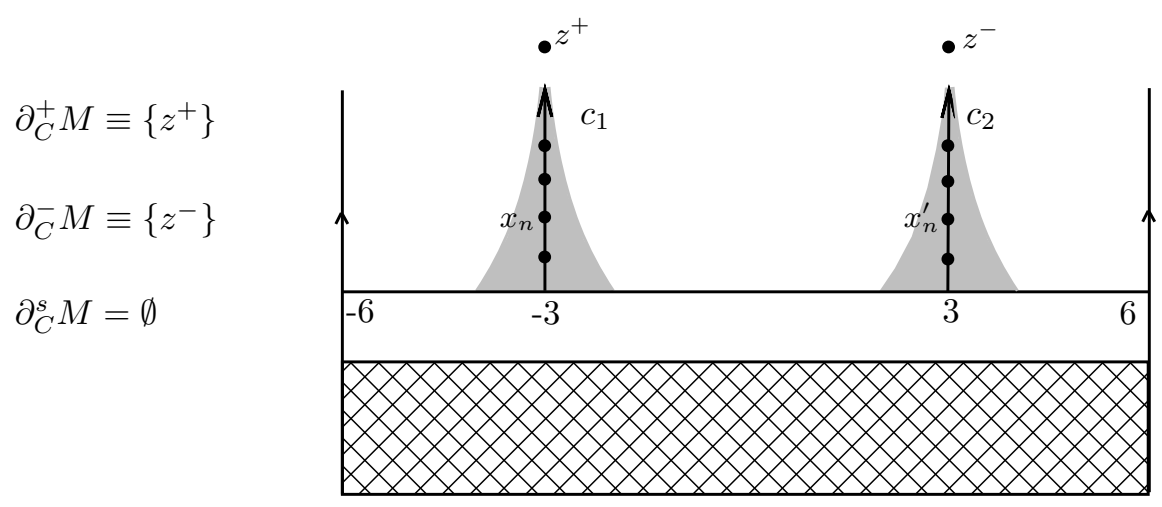

Figure 2: Non-evenly pairing (forward and backward) boundaries, see Remark 3.33 Definition 3.34 .

Starting at a strip of the plane, the two vertical lines at $x= \pm 6$ are identified (the dashed region will be irrelevant). Then, a Randers metric $F=\sqrt{d x^{2}+d y^{2}+\omega^{2}}+\omega$ is chosen such that $c_{1}$ (resp. $c_{2}$ ) determines the unique forward (resp. backward) boundary point $z^{+} \in \partial_{C}^{+} M$ (resp. $z^{-} \in \partial_{C}^{-} M$ ): namely, the support of $\omega$ is included in the grey region and $\omega\left(\partial_{y}\right)$ decreases (resp. increases) strictly to -1 (resp. 1) on $c_{1}$ (resp. $c_{2}$ ) so that the $F$-length of $c_{1}$ and $-c_{2}$ (the reversed parametrization of $c_{2}$ ) becomes finite but the $F$-length of $-c_{1}$ and $c_{2}$ is infinite. The distance $d\left(z^{+}, z^{-}\right)$ is finite - essentially, one could use horizontal lines to connect the points in the Cauchy sequence $\left\{x_{n}\right\}$ which defines $z^{+}$with the points in the Cauchy sequence $\left\{x_{n}^{\prime}\right\}$ which defines $z^{-}$. Moreover, $d\left(z^{+}, x\right), d\left(x, z^{-}\right)$are finite too for any $x \in M$ - say, for large $n$, go from $x_{n}$ to $c_{2}$ by using a horizontal line, go down by using $c_{2}$ until the height of $x$, and use again an horizontal segment to reach $x$. However, $z^{+}, z^{-}$do not belong to $\partial_{C}^{s} M$ (in fact, $\partial_{C}^{s} M=\emptyset$ ).

may be non-connectable to any $x \in M$ by means of a curve of finite length, but all the elements of $\left\{x_{n}\right\}$ may be connectable with $x$ by means of a sequence of curves of bounded length. Figure 2 shows an example of this situation.

In spite of examples as the illustrated one in Figure 2, the length spaces satisfying that any two points $x^{+}, x$ such that $d\left(x^{+}, x\right)<\infty$ admit a curve of finite length from $x^{+}$to $x$ (and thus, $x^{+} \in \partial_{C}^{s} M$ ), constitute a natural subclass: say, as any point $x^{+}$of the boundary and any point $x$ in $M$ can be connected by means of some curve in $M$ (as explained in the previous remark), if $d\left(x^{+}, x\right)<\infty$, one may expect that such a curve can be chosen of finite length. The following definition includes, in particular, this class.

Definition 3.34 A generalized metric space $(M, d)$ is said to have forward (resp. backward) evenly pairing boundary if the following condition holds:

$$
\begin{array}{rlll}
d\left(x^{+}, x\right)=\infty & \forall x^{+} \in \partial_{C}^{+} M \backslash \partial_{C}^{s} M & \text { for some, and then any, } x \in M_{C}^{-} \\
\text {(resp. } \quad d\left(x, x^{-}\right)=\infty & \forall x^{-} \in \partial_{C}^{-} M \backslash \partial_{C}^{s} M & \text { for some, and then any, } \left.x \in M_{C}^{+}\right) .
\end{array}
$$


We have emphasized in this definition that, when the first (resp. second) condition is satisfied for some $x \in M$, then it holds for any $x \in M_{C}^{-}$(resp. $x \in M_{C}^{+}$). So, in the practice, it suffices to check them for some point of $M$. For example, if Figure 2 were modified so that the support of $\omega$ became included in only one of the two grey regions, the boundaries would be clearly evenly pairing. Trivially, from Proposition 3.28

Corollary 3.35 If $d_{Q}$ is a generalized distance, then $(M, d)$ has forward and backward evenly pairing boundaries.

\section{Riemannian Gromov and Busemann completions}

Eberlein and O'Neill [9] developed a well-known compactification for any Hadamard manifold (or, in general, any CAT(0)-space), based in classes of equivalence of rays. Gromov [17] introduced a universal compactification for any complete Riemannian manifold, valid also for some more general complete metric spaces [17, p. 184f]. The latter compactification becomes equivalent to the former in Hadamard manifolds; in order to prove this, Busemann functions play an essential role (see, for example, the books [2, [5]). In this paper, we are interested in analogous boundaries for arbitrary Riemannian and Finslerian manifolds.

We start by reviewing briefly Gromov's construction for any Riemannian manifold, including the non-complete case, with a threefold aim. First, to make clear in which sense the Cauchy boundary can be regarded as a part of Gromov's one. Second, to show the appearance of the Busemann boundary as an intermediate boundary between the Cauchy and Gromov's ones. And third, to introduce the precise framework to be used in the Finslerian case and the remainder of the paper.

Our objective is to study a metric space $\left(M, d^{R}\right)$, being $M$ a (connected) smooth manifold and $d^{R}$ the distance associated to a Riemannian metric $g_{R}$ on $M$. However, the results will be stated in a more general setting, concretely:

Convention 4.1 Along the present Section $4 .\left(M, d^{R}\right)$ will denote any metric space which satisfies: $M$ is locally compact with a countable dense set (or equivalently, which is second countable), and $d^{R}$ is derived from a length space. Recall that the metric space associated to any reversible Finsler manifold is included. Typical notations in manifolds are extended to this general setting with no further mention. In particular, if $c$ is a smooth curve, the role of Riemannian norm $|\dot{c}(t)|=\sqrt{g_{R}(\dot{c}(t), \dot{c}(t))}$ in a general length space will be played by the dilatation. In general, we will adopt the terminology in [16, Chapter 1].

Remark 4.2 As above, $\partial_{C} M$ denotes the Cauchy boundary, and $M_{C}$ the Cauchy completion, the latter endowed with the continuous extension of $d^{R}$ (denoted again by $\left.d^{R}\right)$. Notice that $\left(M_{C}, d^{R}\right)$ is clearly a length space, but it may not lie under Convention 4.1 as $M_{C}$ may not be locally compact, even if $\left(M, d^{R}\right)$ comes from a Riemannian manifold. 


\subsection{Gromov completion}

For each $x \in M_{C}$, let $d_{x}^{R}$ be the Lipschitz function on $M$ given by $y \mapsto d^{R}(y, x)$. Consider the space $\mathcal{L}_{1}\left(M, d^{R}\right)$ of all the Lipschitz functions (always assumed with Lipschitz constant equal to 1 ) on $M$, endowed with the topology of pointwise convergence. Notice that this space is equivalent to the space of Lipschitz functions on $M_{C}$; in particular, functions $f \in \mathcal{L}_{1}\left(M, d^{R}\right)$ will be extended to $M_{C}$ with no further mention.

Remark 4.3 The topology of pointwise convergence on $\mathcal{L}_{1}\left(M, d^{R}\right)$ coincides with the topology of uniform convergences on compact sets and the compact-open topology; moreover, it is metrizable. In fact, consider the space $\mathcal{C}(M)$ of all the continuous functions from $M$ to $\mathbb{R}$. In this space the topology of uniform convergence on compact sets coincides with the compact-open topology (see [24, p. 230]) and, when restricted to $\mathcal{L}_{1}\left(M, d^{R}\right)$, with the pointwise convergence topology (see [24, p. 232]). It is not hard to prove that $\mathcal{C}(M)$ is Hausdorff, regular and second countable (as so are $M$ and $\mathbb{R})$. So, by Urysohn theorem, $\mathcal{C}(M)$, and then $\mathcal{L}_{1}\left(M, d^{R}\right)$, are metrizable. Even more, the following metric $d^{1}$ on $\mathcal{L}_{1}\left(M, d^{R}\right)$ generates the topology. Choose $x_{0} \in M$ and an auxiliary complete metric $\tilde{d}^{R}$ such that $\tilde{d}^{R} \geq d^{R}$, and define the metric

$$
d^{1}\left(f_{1}, f_{2}\right):=\sup _{x \in M} \frac{\left|f_{1}(x)-f_{2}(x)\right|}{1+\tilde{d}^{R}\left(x, x_{0}\right)^{2}} \quad \forall f_{1}, f_{2} \in \mathcal{L}_{1}\left(M, d^{R}\right) .
$$

Let $f_{n}, f \in \mathcal{L}_{1}\left(M, d^{R}\right), n \in \mathbb{N}$. Clearly, if $\left\{d^{1}\left(f_{n}, f\right)\right\}$ converges to 0 , then the sequence $\left\{f_{n}\right\}$ converges pointwise to $f$. For the converse, the Lipschitz condition yields the following bound for the expression corresponding to the fraction in 4.1):

$$
\frac{\left|f\left(x_{0}\right)\right|+\left|f_{n}\left(x_{0}\right)\right|+2 d^{R}\left(x, x_{0}\right)}{1+\tilde{d}^{R}\left(x, x_{0}\right)^{2}} \leq 2 \frac{1+\left|f\left(x_{0}\right)\right|+\tilde{d}^{R}\left(x, x_{0}\right)}{1+\tilde{d}^{R}\left(x, x_{0}\right)^{2}},
$$

(the last inequality for large $n$ ). So, the uniform convergence of $\left\{f_{n}\right\}$ on $\tilde{d}^{R}$-bounded sets completes the result.

Lemma 4.4 Let $\left\{f_{n}\right\}$ be a sequence in $\mathcal{L}_{1}\left(M, d^{R}\right)$. Assume that there exists $x_{0} \in$ $M_{C}$ such that $\left\{f_{n}\left(x_{0}\right)\right\}$ converges to some $k_{0} \in[-\infty, \infty]$. If $k_{0} \in \mathbb{R}$ then $\left\{f_{n}\right\}$ admits a subsequence converging in $\mathcal{L}_{1}\left(M, d^{R}\right)$. If $k_{0}= \pm \infty$ then $\left\{f_{n}\right\}$ converges uniformly on compact sets to $\pm \infty$.

Proof. Only the case $k_{0} \in \mathbb{R}$ will be considered, as $k_{0}= \pm \infty$ is similar.

Let $\left\{x_{n}\right\}_{n=0}^{\infty} \subset M$ be a countable dense subset of $M$. Remove a finite subset of $\left\{f_{n}\right\}$, to obtain a subsequence $\left\{f_{n}^{0}\right\}$ with $\left|f_{n}^{0}\left(x_{0}\right)-k_{0}\right|<1$ for all $n$. From the Lipschitz condition, one has

$$
-d^{R}\left(x_{0}, x_{m}\right)-1 \leq f_{n}^{0}\left(x_{m}\right)-k_{0} \leq d^{R}\left(x_{0}, x_{m}\right)+1 \quad \forall n, m .
$$

Now, we can construct inductively a subsequence $\left\{f_{n}^{m}\right\}_{n}$ of $\left\{f_{n}^{m-1}\right\}_{n}$ such that $\left\{f_{n}^{m}\left(x_{i}\right)\right\}_{n}$ is convergent for $i=1, \ldots, m$. Then, the diagonal subsequence $\left\{f_{n}^{n}\right\}_{n}$ is convergent in $\mathcal{L}_{1}\left(M, d^{R}\right)$, as it converges on each $x_{m}$. 
Proposition 4.5 The mar

$$
\hat{j}: M_{C} \rightarrow \mathcal{L}_{1}\left(M, d^{R}\right), \quad x \mapsto-d_{x}^{R}
$$

is a topological embedding of $M_{C}$ in $\mathcal{L}_{1}\left(M, d^{R}\right)$.

If $K \subset M_{C}$ is any bounded subset then the closure of $\hat{j}(K)$ in $\mathcal{L}_{1}\left(M, d^{R}\right)$ is compact.

Proof. The first assertion is standard (simplify Lemma 5.5 below). For the last one, we only have to show that the closure of $\hat{j}(K)$ is sequentially compact, since $\mathcal{L}_{1}\left(M, d^{R}\right)$ is metrizable by Remark 4.3 By Lemma 4.4 . it suffices to check that any sequence $\left\{f_{n}\right\}$ in the closure of $\hat{j}(K)$ admits some $x \in M_{C}$ such that $\left\{\left|f_{n}(x)\right|\right\}$ is bounded. Notice that $f_{n}=\lim _{i} \hat{j}\left(y_{n}^{i}\right)$ for some sequence $\left\{y_{n}^{i}\right\}_{i=0}^{\infty} \subset K$. Hence, for any $x \in M_{C}$ :

$$
\begin{aligned}
\left|f_{n}(x)-\left(\hat{j}\left(y_{n}^{0}\right)\right)(x)\right| & =\left|\lim _{i}\left(\hat{j}\left(y_{n}^{i}\right)\right)(x)-\left(\hat{j}\left(y_{n}^{0}\right)\right)(x)\right| \\
& \leq \lim _{i}\left|d^{R}\left(x, y_{n}^{i}\right)-d^{R}\left(x, y_{n}^{0}\right)\right| \leq \lim _{i} d^{R}\left(y_{n}^{0}, y_{n}^{i}\right) .
\end{aligned}
$$

But the last term is bounded by the diameter of $K$, as required.

$\mathcal{L}_{1}\left(M, d^{R}\right)_{*}=\mathcal{L}_{1}\left(M, d^{R}\right) / \mathbb{R}$ will be the topological quotient of $\mathcal{L}_{1}\left(M, d^{R}\right)$ by the 1-dimensional subspace of the (real) constant functions. The natural quotient topology will be called Gromov or (quotient) pointwise topology:

Proposition 4.6 The composition map

$$
j: M_{C} \rightarrow \mathcal{L}_{1}\left(M, d^{R}\right)_{*}, \quad x \mapsto\left[-d_{x}^{R}\right]
$$

is continuous and injective.

Moreover, the restriction $\left.j\right|_{M}: M \rightarrow \mathcal{L}_{1}\left(M, d^{R}\right)_{*}$ is a topological embedding.

Proof. The continuity of $j$ follows from the continuity of $\hat{j}$, and the injectivity from the following property: if $x \neq y$ then $d_{x}^{R}-d_{y}^{R}$ changes its sign at $x, y$, and so, cannot be constant. So, one only has to prove the continuity of $j^{-1}$ on $j(M)$.

To this aim, let $x_{n}, x \in M$ such that $\left\{j\left(x_{n}\right)\right\} \rightarrow j(x)$. Then, there exists a sequence $\left\{t_{n}\right\} \subset \mathbb{R}$ such that $\left\{t_{n}-d_{x_{n}}^{R}\right\}_{n} \rightarrow-d_{x}^{R}$ with the pointwise topology. Assume by contradiction that $\left\{x_{n}\right\} \nrightarrow \rightarrow x$. Up to a subsequence, there exists $\epsilon>0$ small enough such that $x_{n} \notin \overline{B(x, 2 \epsilon)}$ for all $n$. We can also assume that the spheres $S(x, r):=\left\{y \in M: d^{R}(y, x)=r\right\}$ are compact for $r=\epsilon, 2 \epsilon$. Notice that, for any $y \in S(x, 2 \epsilon)$ and $z \in S(x, \epsilon)]^{4}$

$$
\begin{aligned}
\lim _{n}\left(d^{R}\left(z, x_{n}\right)-d^{R}\left(y, x_{n}\right)\right) & =\lim _{n}\left(t_{n}-d^{R}\left(y, x_{n}\right)\right)-\lim _{n}\left(t_{n}-d^{R}\left(z, x_{n}\right)\right) \\
& =-d^{R}(y, x)+d^{R}(z, x)=-\epsilon<0 .
\end{aligned}
$$

\footnotetext{
${ }^{3}$ For convenience, we change the sign of the natural definition here. Later, this will affect only to the sign of Busemann functions, which will be the opposite of the one in the usual convention.

${ }^{4}$ The first equality is justified by the existence of a finite limit stated in the second one. We will use such a posteriori justifications with no further mention.
} 
On the other hand, for each $n$ we can choose $y_{n} \in S(x, 2 \epsilon)$ and $z_{n} \in S(x, \epsilon)$ such that

$$
d^{R}\left(z_{n}, x_{n}\right)-d^{R}\left(y_{n}, x_{n}\right) \geq 0 .
$$

In fact, consider a sequence of curves $\gamma_{n}^{j}$ from $x$ to $x_{n}$ with $\left\{\operatorname{length}\left(\gamma_{n}^{j}\right)\right\}_{j} \rightarrow$ $d^{R}\left(x, x_{n}\right)$. Each $\gamma_{n}^{j}$ will intersect $S(x, 2 \epsilon), S(x, \epsilon)$ in some points $y_{n}^{j}, z_{n}^{j}$, resp., and, with no loss of generality, one can assume the convergence $\left\{y_{n}^{j}\right\}_{j} \rightarrow y_{n}$ and $\left\{z_{n}^{j}\right\}_{j} \rightarrow z_{n}$. Then, by the continuity of $d^{R}(\cdot, x)$, such points $\left\{y_{n}\right\}$ and $\left\{z_{n}\right\}$ satisfy $d^{R}\left(z_{n}, x_{n}\right)=d^{R}\left(z_{n}, y_{n}\right)+d^{R}\left(y_{n}, x_{n}\right) \geq d^{R}\left(y_{n}, x_{n}\right)$. Finally, the compactness of the spheres allows to assume that $\left\{y_{n}\right\},\left\{z_{n}\right\}$ converge, obtaining a contradiction with 4.3 , 4.2.

Remark 4.7 Notice that when $M_{C}$ is locally compact, it also lies under Convention 4.1 (Remark 4.2), and so, the proof of Proposition 4.6 also ensures that $j$ is an embedding on all $M_{C}$.

The following technical result will be useful for future referencing.

Lemma 4.8 Consider $j: M_{C} \rightarrow \mathcal{L}_{1}\left(M, d^{R}\right)_{*}$ and $x_{n}, x \in M_{C}$. If $\left\{\left[-d_{x_{n}}^{R}\right]\right\}_{n} \rightarrow$ $\left[-d_{x}^{R}\right]$ then

$$
\lim _{n}\left(d^{R}(\cdot, x)+d^{R}\left(x, x_{n}\right)-d^{R}\left(\cdot, x_{n}\right)\right)=0 .
$$

Proof. The limit $\left\{\left[-d_{x_{n}}^{R}\right]\right\}_{n} \rightarrow\left[-d_{x}^{R}\right]$ provides the existence of $\left\{t_{n}\right\}$ such that $\left\{t_{n}-\right.$ $\left.d^{R}\left(\cdot, x_{n}\right)\right\}_{n}$ converges pointwise to $-d^{R}(\cdot, x)$. Then, if we evaluate this expression at $x$, we deduce $t_{n}-d^{R}\left(x, x_{n}\right) \rightarrow 0$. Therefore,

$$
\begin{gathered}
\lim _{n}\left(d^{R}(\cdot, x)+d^{R}\left(x, x_{n}\right)-d^{R}\left(\cdot, x_{n}\right)\right) \\
\quad=d^{R}(\cdot, x)-\lim _{n}\left(t_{n}-d^{R}\left(x, x_{n}\right)\right)+\lim _{n}\left(t_{n}-d^{R}\left(\cdot, x_{n}\right)\right) \\
=d^{R}(\cdot, x)-d^{R}(\cdot, x)=0
\end{gathered}
$$

Example 4.9 Here we are going to use bounded (i.e. without "directions at infinity") examples to illustrate the following four properties: (a) $j$ may be not an embedding on all $M_{C}$ and $\hat{j}\left(M_{C}\right)$ may be non-closed in $\mathcal{L}_{1}\left(M, d^{R}\right)$; (b) even if $j$ is a topological embedding, $j\left(M_{C}\right)$ may be non-closed in $\mathcal{L}_{1}\left(M, d^{R}\right)_{*} ;(\mathrm{c})$ even if $j\left(M_{C}\right)$ is closed, the map $j: M_{C} \rightarrow \mathcal{L}_{1}\left(M, d^{R}\right)_{*}$ may not be a topological embedding; and (d) even if $j$ is a topological embedding, $M_{C}$ may not be locally compact. These items will be illuminating to understand the way in which the Cauchy boundary lies in the Gromov one, and they also show the optimal character of Propositions 4.5 and 4.6 .

Consider the metric space $\left(M, d^{R}\right)$ with

$$
M:=\cup_{n=1}^{\infty}\left\{\left(n^{-1}, y\right): y \in[0,1)\right\} \cup\{(x, 0): x \in(0,1]\}
$$




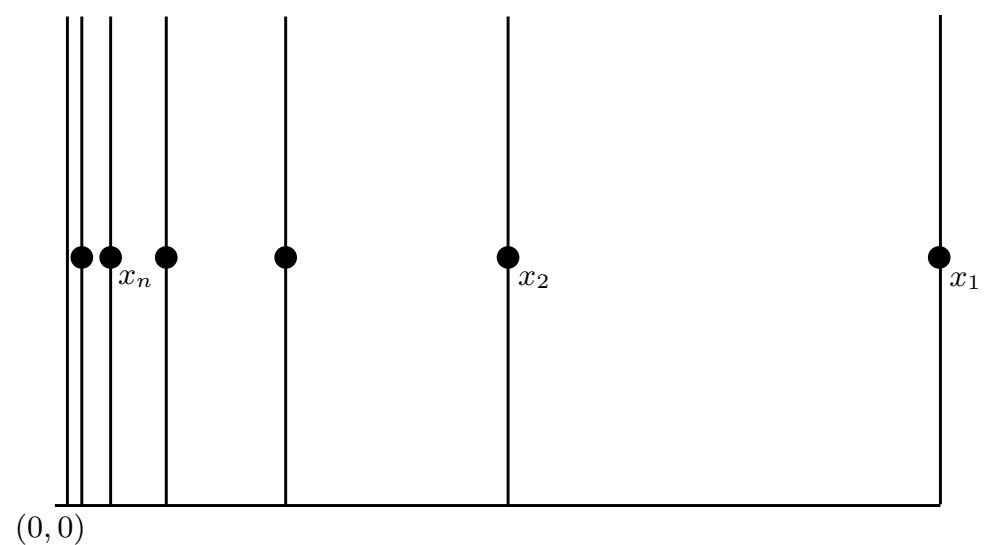

Figure 3: $\quad M_{G}$ and $j\left(M_{C}\right)$ coincide as point sets but not topologically.

Both, Gromov and Cauchy completions include naturally $(0,0)$ as a boundary point, and coincide as point sets. However, $\left\{x_{n}\right\}$ converges to $(0,0)$ only for Gromov topology. Moreover, $M_{C}$ is not locally compact, and $j: M_{C} \rightarrow M_{G}$ is continuous but not a topological embedding.

and $d^{R}(x, y)$ obtained as the infimum of the usual lengths of piecewise smooth curves in $M$ connecting $x$ and $y$ (see Fig. 3$]^{5}$. The sequences type $\left\{\left(n^{-1}, y_{0}\right)\right\}_{n}$ with $0<$ $y_{0}<1$ do not contain any convergent subsequence in $M_{C}$; in fact, $\partial_{C} M$ is naturally identifiable to $(0,0)$ plus the non-Cauchy sequence $\left\{\left(n^{-1}, 1\right)\right\}_{n=1}^{\infty}$. However, for any $0 \leq y_{0} \leq 1$, each sequence $\left\{-d_{\left(n^{-1}, y_{0}\right)}^{R}\right\}_{n}$ converges to a function in $\mathcal{L}_{1}\left(M, d^{R}\right)$, which will be denoted $-d_{\left(0, y_{0}\right)}^{R}$-notice that it corresponds with the natural (minus) distance to $\left(0, y_{0}\right)$ in the closure of $M$ as a subset of $\mathbb{R}^{2}$. In particular, this shows (a).

For (c), note that the sequence of functions $\left\{y_{0}-d_{\left(n^{-1}, y_{0}\right)}^{R}\right\}_{n}, y_{0} \in[0,1]$, converges pointwise to $-d_{(0,0)}^{R}$. So, the sequence of classes $\left\{\left[-d_{\left(n^{-1}, y_{0}\right)}^{R}\right]\right\}$ converges to $\left[-d_{(0,0)}^{R}\right]$ in $\mathcal{L}_{1}\left(M, d^{R}\right)_{*}$ (intuitively, $\left.\left[-d_{(0,0)}^{R}\right]=\left[-d_{\left(0, y_{0}\right)}^{R}\right]\right)$, and $j\left(M_{C}\right)$ is closed.

For (b) and (d), modify slightly previous example by enlarging it and redefining $M$ as:

$$
M:=M^{\prime} \cup\{(x, 1): x \in(0,1]\}
$$

where $M^{\prime}$ is now given by the expression in 4.4 (see Fig. 4(A)). Observe that $j$ is a topological embedding. In fact, from Prop. 4.6 we only have to check the continuity of $\left.j^{-1}\right|_{j\left(M_{C}\right)}$. Observe that if $\left\{\left[-d_{x_{n}}^{R}\right]\right\}_{n}$ converges to $\left[-d_{x}^{R}\right]$ in $\mathcal{L}_{1}\left(M, d^{R}\right)_{*}$ for $x_{n}, x \in M_{C}$, Lemma 4.8 ensures that $\lim _{n}\left(d^{R}(\cdot, x)+d^{R}\left(x, x_{n}\right)-d^{R}\left(\cdot, x_{n}\right)\right)=0$. If $\lim _{n} d^{R}\left(x, x_{n}\right) \neq 0$, one can obtain the following contradiction: there exist $y \in$

\footnotetext{
${ }^{5}$ Notice that $\left(M, d^{R}\right)$ fulfills the essential properties in Convention 4.1 Even though, for simplicity, this space is not a manifold, the example can be easily transformed into a Riemannian 2 -manifold by enlarging each vertical line in a strip (thinner as $n$ grows) and including also the forth quadrant, $x>0, y<0$. Analogously, $M^{\prime}$ in 4.5 below can be also transformed into a manifold.
} 
$M, \epsilon>0$ and a subsequence $\left\{x_{n_{k}}\right\}$ such that $d^{R}(y, x)+d^{R}\left(x, x_{n_{k}}\right)>d^{R}\left(y, x_{n_{k}}\right)+\epsilon$. In fact, considering $\left\{x_{n}\right\}$ as a sequence in $\mathbb{R}^{2}$, it will converge, up to a subsequence, to some $x_{0} \in \mathbb{R}^{2}$ (with $x_{0} \neq x$ ) such that $x_{0} \in M_{C}$. Depending on the relative position of $x_{0}$ and $x$, the claimed $y$ is chosen such that the strict triangle inequality $d^{R}(y, x)+d^{R}\left(x, x_{0}\right)>d^{R}\left(y, x_{0}\right)$ holds.

Observe that, for $x_{n}=\left(n^{-1}, 1 / 2\right)$, the sequence $\left\{\left[-d_{x_{n}}^{R}\right]\right\}$ converges in $\mathcal{L}_{1}\left(M, d^{R}\right)_{*}$ to the class of the function $\max \left\{-d_{(0,0)}^{R},-d_{(0,1)}^{R}\right\}$ (see Fig. 4 (B)), which does not correspond with any element of $M_{C}$. This proves (b) and, for (d), recall that $M_{C}$ is not locally compact, as any neighborhood of $(0,0)$ contains a sequence of the form $\left\{\left(n^{-1}, \epsilon\right)\right\}$ for $\epsilon>0$ small enough, and this sequence does not converge in $M_{C}$.

Definition 4.10 Let $\left(M, d^{R}\right)$ be the metric space associated to a Riemannian manifold (or any space under Convention 4.1).

The Gromov completion $M_{G}$ of $\left(M, d^{R}\right)$ is the closure of $j(M)$ in $\mathcal{L}_{1}\left(M, d^{R}\right)_{*}$, and the Gromov boundary $\partial_{G} M$ is $\partial_{G} M:=\overline{j(M)} \backslash j(M)$.

The map obtained by restricting the co-domain of the map $j$ defined in Prop. 4.6 is also denoted

$$
j: M_{C} \rightarrow M_{G},
$$

and shows that the Cauchy boundary can be regarded as a point set included in the Gromov one.

Remark 4.11 Typically in Gromov's approach, one considers, instead of $\mathcal{L}_{1}\left(M, d^{R}\right)$, the space $\mathcal{C}(M)$ of all the continuous functions on $M$ endowed with the topology of uniform convergence on compact sets. However, in order to compute the closure of $j(M)$, this difference in the ambient space becomes irrelevant. In fact, if $[h]$ belongs to the closure of $j(M)$ in $\mathcal{C}(M)_{*}$ then any $h \in[h]$ belongs to $\mathcal{L}_{1}\left(M, d^{R}\right)$. Moreover, on this space, the topology of uniform convergence on compact sets and the topology of pointwise convergence coincide (see Remark 4.3).

Theorem 4.12 The Gromov completion $M_{G}$ of any metric space $\left(M, d^{R}\right)$ associated to a Riemannian metric (or space under Convention 4.1) is a compact metrizable space.

The image of the manifold $j(M) \subset M_{G}$ is an open dense subset of $M_{G}$ and, thus, the boundary $\partial_{G} M$ is also compact.

Proof. Take some $x_{0} \in M$. The map $i: \mathcal{L}_{1}\left(M, d^{R}\right)_{*} \rightarrow \mathcal{L}_{1}\left(M, d^{R}\right)$ which maps each class $[f]$ to its representative $f_{0}$ such that $f_{0}\left(x_{0}\right)=0$, is a topological embedding. On the other hand, by Lemma $4.4 . i\left(\mathcal{L}_{1}\left(M, d^{R}\right)_{*}\right)$ is sequentially compact. Hence, the metrizable space $\mathcal{L}_{1}\left(M, d^{R}\right)_{*}$ is also sequentially compact, and thus compact (see, for example, 28, Theor. 28.2]). So, the first assertion follows from the fact that $M_{G}$ is closed in $\mathcal{L}_{1}\left(M, d^{R}\right)_{*}$. Finally, the density of $j(M)$ follows trivially from the construction of $M_{G}$, and its openness from the local compactness of $M$ as in Proposition 3.30 (see also Corollary 5.25 below).

As $\left.j\right|_{M}$ is an embedding, the continuous map $j$ can be dropped for $M$, and we will write simply $M \subset M_{G}$. However, $j$ will be maintained for $M_{C}$ and $\partial_{C} M$, as 


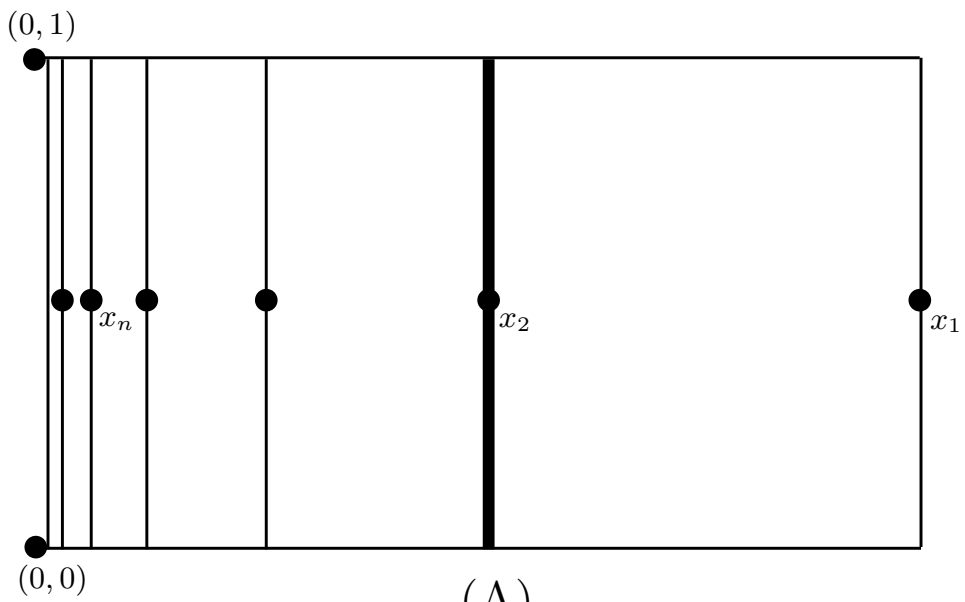

(A)

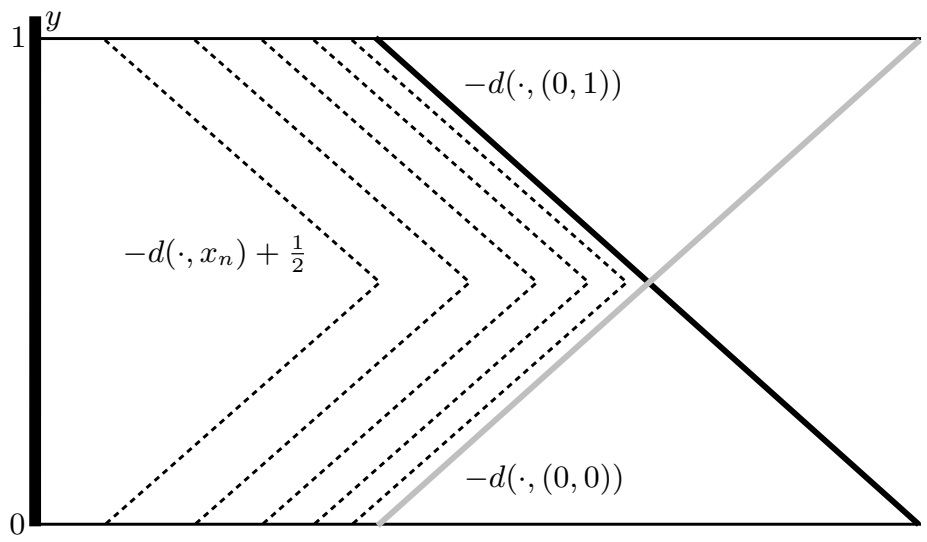

(B)

Figure 4: $\quad$ Bounded example with $j\left(M_{C}\right)$ non-closed in $\mathcal{L}_{1}\left(M, d^{R}\right)_{*}$ (Example 4.9 (b)) and non-Hausdorff chr. topology (Remark 5.30).

(A) $\partial_{C} M$ includes only $(0,0)$ and $(0,1)$, but $\partial_{G} M$ also includes in a natural way the other points $S=\{(0, y): 0<y<1\}$ of the segment between that points. Then, $j: M_{C} \rightarrow M_{G}$ is an embedding but $j\left(M_{C}\right)$ is not closed and $j\left(\partial_{C} M\right) \subsetneq \partial_{C G} M$. Moreover, the point in $\partial_{G} M$ defined by the sequence $\left\{x_{n}\right\}_{n}$ (or any other point in $\left.S \subset \partial_{G} M\right)$ cannot be reached as the limit of any curve in $M$.

(B) Visualization of the chr. convergence of $\left\{\left[-d\left(\cdot, x_{n}\right)\right]\right\}_{n}$ to $[-d(\cdot,(0,0))]$ and $[-d(\cdot,(0,1))]$ in Figure $(\mathrm{A})$. The bold vertical axis represents the vertical segment through $x_{2}$ in (A). The dashed lines correspond to the graph of the functions $-d\left(\cdot, x_{n}\right)+1 / 2$ on that vertical segment. The limit is $\max \{-d(\cdot,(0,0)),-d(\cdot,(0,1))\}$. 
$j\left(M_{C}\right), j\left(\partial_{C} M\right)$ will be endowed with the restriction of the Gromov topology of $M_{G}$, which, in general, does not agree with the Cauchy completion topology (Example 4.9. property (c)). In the Gromov boundary $\partial_{G} M$, one can distinguish two disjoint parts: the Cauchy-Gromov boundary $\partial_{C G} M$, which consists of all the points which can be obtained as the limit of some sequence of $M$ included in some bounded subset, and the proper Gromov boundary $\partial_{\mathcal{G}} M=\partial_{G} M \backslash \partial_{C G} M$, which corresponds with the "boundary at infinity". The Cauchy boundary $\partial_{C} M$ is naturally included in $\partial_{C G} M$ and, as Example 4.9 (property (b)) shows, the inclusion can be strict, i.e. a residual Gromov boundary $\partial_{R G} M=\partial_{C G} M \backslash j\left(\partial_{C} M\right)$ may appear. Summing up:

$$
M_{G}=M \cup \partial_{G} M \quad \partial_{G} M=\partial_{C G} M \cup \partial_{\mathcal{G}} M \quad \partial_{C G} M=j\left(\partial_{C} M\right) \cup \partial_{R G} M .
$$

The topological subspace $M_{C G}:=M \cup \partial_{C G} M$ of $M_{G}$ will be called the CauchyGromov completion and its properties are summarized as follows.

Corollary 4.13 The Cauchy-Gromov completion $M_{C G}$ satisfies:

(1) (Openness of $M$ ). The boundary $\partial_{C G} M$ is closed in $M_{C G}$.

(2) (Heine-Borel). The closure in $M_{C G}$ of any bounded set in $M$ is compact.

(3) (Consistency with the Cauchy boundary). If $M_{C}$ is locally compact, then $j$ : $M_{C} \rightarrow M_{G}$ is an embedding and $M_{C} \equiv M_{C G}$, i.e. $\partial_{R G} M=\emptyset$.

Proof. The first assertion is straightforward from Theorem 4.12, For the second assertion recall that, from the compactness of $M_{G}$, the closure of any set in $M_{G}$ is compact and, from the definition of $M_{C G}$, the closure of any bounded set belongs to $M_{C G}$. The first statement in (3) follows from Remark 4.7 and, for the last statement, it suffices to show that, if $M_{C}$ is locally compact, then the closed balls in $M_{C}$, $\overline{B\left(x, r ; M_{C}\right)}, x \in M_{C}, r>0$, are compact. In fact, in this case any sequence defining a point of the Cauchy-Gromov boundary will have a convergent subsequence in $M_{C}$, and the result follows from the continuity of $j$ and the Hausdorffness of $M_{G}$.

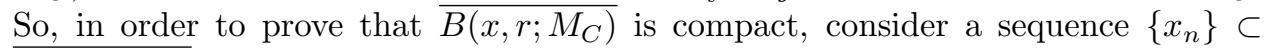
$\overline{B\left(x, r ; M_{C}\right)}$. Define a sequence of curves $\left\{\gamma_{n}\right\}, \gamma_{n}:[0,1] \rightarrow M$ joining $x$ and $x_{n}$, such that: each $\gamma_{n}$ restricted to $(0,1)$ is smooth and contained in $M$, it is parametrized with constant speed and length $\left(\gamma_{n}\right)<2 r$ (see Remark 3.12). Clearly, the sequence of curves $\left\{\gamma_{n}\right\}_{n}$ is equicontinuous ${ }^{6}$. Taking into account that $x$ is an accumulation point of the sequence $\left\{\gamma_{n}(0)\right\}$, and $M_{C}$ is both, locally compact and complete, classical Arzela's Theorem (see for example, Theorems 5.12 5.15 below) implies the existence of a curve $\gamma_{\infty}$ which is the pointwise limit of a subsequence of $\left\{\gamma_{n}\right\}$. So, the required limit is $\gamma_{\infty}(1)$.

\footnotetext{
${ }^{6}$ For posterior referencing, note that, if $t_{1}<t_{2}$ then $d^{R}\left(\gamma_{n}\left(t_{1}\right), \gamma_{n}\left(t_{2}\right)\right) \leq\left.\operatorname{length}\left(\gamma_{n}\right)\right|_{\left[t_{1}, t_{2}\right]}=$ $\left(t_{2}-t_{1}\right) \operatorname{length}\left(\gamma_{n}\right)<\left(t_{2}-t_{1}\right) 2 r$. This inequality, plus the symmetry of $d^{R}$, implies the equicontinuity.
} 


\subsection{Busemann completion as a point set}

Next, we are going to focus our attention on certain subset of $M_{G}$, which provides another compactification of $\left(M, d^{R}\right)$ when is endowed with a different topology.

Let $C(M)$ be the space of all the piecewise smooth curves $c:[\alpha, \Omega) \rightarrow M$, $-\infty<\alpha<\Omega \leq \infty$, with $|\dot{c}|^{2}=g_{R}(\dot{c}, \dot{c})<1$ and consider the associated function:

$$
b_{c}(x)=\lim _{s \nearrow \Omega}\left(s-d^{R}(x, c(s))\right) \in \mathbb{R} \cup\{+\infty\}, \quad x \in M .
$$

The following result shows that $b_{c}$ is well-defined:

Lemma 4.14 If $c \in C(M)$ then the map $s \mapsto s-d^{R}(x, c(s))$ is increasing for any $x \in M$.

Proof. Just notice:

$$
d^{R}\left(x, c\left(s_{2}\right)\right)-d^{R}\left(x, c\left(s_{1}\right)\right) \leq d^{R}\left(c\left(s_{1}\right), c\left(s_{2}\right)\right) \leq \operatorname{length}\left(\left.c\right|_{\left[s_{1}, s_{2}\right]}\right)<s_{2}-s_{1} .
$$

Proposition 4.15 Let $b_{c}$ be associated to some $c \in C(M)$.

(1) If $b_{c}$ reaches the value $+\infty$ at some $x \in M$ then $b_{c} \equiv \infty$; otherwise, $b_{c} \in$ $\mathcal{L}_{1}\left(M, d^{R}\right)$ and its corresponding class $\left[b_{c}\right]$ belongs to $M_{G}$.

(2) If $\Omega<\infty$ then: (i) there exists $\bar{x} \in M_{C}$ such that $\lim _{s} \nearrow_{\Omega} c(s)=\bar{x}$ in the metric topology of $M_{C}$; (ii) $b_{c}(\cdot)=\Omega-d_{\bar{x}}^{R}(\cdot)$; (iii) $j(\bar{x})=\left[b_{c}\right] \in j\left(M_{C}\right)\left(\subset M_{G}\right)$ and $\lim _{s} \Omega_{\Omega} j(c(s))=\left[b_{c}\right]$.

Conversely, for any $\bar{x} \in M_{C}$, there exists some $c \in C(M)$ with $\Omega<\infty$ such that $j(\bar{x})=\left[b_{c}\right]$. In particular, for $x \in M$, one has $j(x)=\left[b_{c_{x}}\right]$, where $c_{x}:[-1,0) \rightarrow M, s \mapsto x$.

(3) If $\Omega=\infty$ and $b_{c} \not \equiv \infty$, then $\left[b_{c}\right] \in \partial_{\mathcal{G}} M$.

Proof. It is a consequence of previous definitions (see also [20, Sect. 2]):

(1) Both assertions follow from $d^{R}(x, c(s))-d^{R}(y, c(s)) \leq d^{R}(x, y)$ for all $s$.

(2) As the length of $c$ is finite, some endpoint $\bar{x} \in M_{C}$ is determined (recall Remark 3.12 . This proves (i), and the pointwise convergence of $d_{c(s)}^{R}$ to $d_{\bar{x}}^{R}$ proves (ii). The remainder follows from the definitions above.

(3) As $b_{c}<\infty$ and $\Omega=\infty$, the curve $c$ must leave any bounded subset of $M$, and so, its class cannot belong to $M_{C G}$.

Definition 4.16 $A$ Busemann function $b: M \rightarrow(-\infty, \infty]$ is a function which can be written as $b=b_{c}$ for some $c \in C(M)$ as in $(4.6)$. The set of all the finite-valued Busemann functions will be denoted $B(M)\left(\subset \mathcal{L}_{1}\left(M, d^{R}\right)\right)$.

$A$ properly Busemann function $b$ is a finite-valued one which is written as $b=b_{c}$ for some $c \in C(M)$ with $\Omega=\infty$. The set of all the properly Busemann functions will be denoted $\mathcal{B}(M)(\subset B(M))$. 
As a point set, the Busemann completion $M_{B}$ of $\left(M, d^{R}\right)$ is defined as the subset $M_{B}=B(M) / \mathbb{R}$ of the Gromov completion $M_{G}$. The Busemann boundary is then $\partial_{B} M=M_{B} \backslash j(M)$ and the properly Busemann boundary $\partial_{\mathcal{B}} M=\mathcal{B}(M) / \mathbb{R}$ (= $\left.M_{B} \backslash j\left(M_{C}\right) \subset \partial_{\mathcal{G}} M\right)$.

Remark 4.17 About the notion of Busemann function, the following comments are in order:

(1) There is no restriction if, in the definition of $B(M)$, one considers curves in $C(M)$ with unit velocity. In fact, if $\bar{c}$ is the arc-reparametrization of a curve $c \in C(M)$ with $b_{c} \in B(M)$, then $b_{c}=k_{0}+b_{\bar{c}}$, where $k_{0}=\lim _{t}\left(t\right.$-length $\left.\left.\right|_{[\alpha, t]} c\right)$ (which is finite as $k_{0}<\lim _{t}\left(t-d^{R}(c(\alpha), c(t))\right)=b_{c}(c(\alpha))<\infty$ ). So, $\tilde{c}(t)=\bar{c}\left(t-k_{0}\right)$ satisfies $b_{\tilde{c}}=b_{c}$, as required. Therefore, we choose $|\dot{c}|^{2}<1$ only for convenience.

(2) Moreover, according to previous paragraph, one can also consider that the curves in $C(M)$ have velocity lower or equal than one.

(3) In any metric space $(X, d)$, one can say that $b: X \rightarrow(-\infty, \infty]$ is a Busemann function when there exists a sequence $\sigma=\left\{x_{n}\right\}_{n} \subset X$ such that $b=b_{\sigma}$, being $b_{\sigma}(x)=\lim _{n}\left(\sum_{k=1}^{n} d\left(x_{k-1}, x_{k}\right)-d\left(x_{n}, x\right)\right), x \in X$. This avoids the use of length spaces. However, we will need other properties of length spaces, and this general definition will not be considered in this paper.

Summing up, Definition 4.16 and Proposition 4.15 yield the disjoint unions

$$
M_{B}=j\left(M_{C}\right) \cup \partial_{\mathcal{B}} M, \quad \partial_{B} M=j\left(\partial_{C} M\right) \cup \partial_{\mathcal{B}} M
$$

and the relations

$$
\partial_{\mathcal{B}} M \subset \partial_{\mathcal{G}} M, \quad \partial_{B} M \cap \partial_{R G} M=\emptyset
$$

complemented with $\partial_{B} M \subset \partial_{G} M, j\left(\partial_{C} M\right) \subset \partial_{C G} M$, where all the inclusions may be strict (see Figure 5 ).

Remark 4.18 In some sense, $\partial_{\mathcal{B}} M$ represents the set of possible "directions towards infinity". In a Hadamard manifold, $\partial_{C} M=\emptyset$ and $\partial_{\mathcal{B}} M=\partial_{G} M$ (see Corollary 5.40 below); moreover, in this case one can construct $\partial_{\mathcal{B}} M$ by using only rays, instead of all $C(M)$ (in fact, the classical construction by Gromov shows that $M_{G}$, and thus $M_{B}$, can be obtained by using only rays). However, it is not difficult to find even a complete, 1-connected Riemannian manifold where $\partial_{\mathcal{B}} M \varsubsetneqq \partial_{G} M$. This is suggested by the example in Figure 5 (B); a different example is the universal covering of the "grapefruit on a stick", studied in detail in [11, Sect. 2.1].

If $\partial_{B} M \neq \partial_{G} M$ then $M_{B}$ is not compact with the pointwise convergence topology, and the name "Busemann completion or compactification" may seem misleading. However, the natural topology for this completion, the chronological topology (which is suggested by the causal boundary), makes $M_{B}$ and $\partial_{B} M$ sequentially compact (but at the price of being non-Hausdorff). Such a topology is studied below, in the more general (non-necessarily symmetric) Finslerian case, Subsection 5.2 


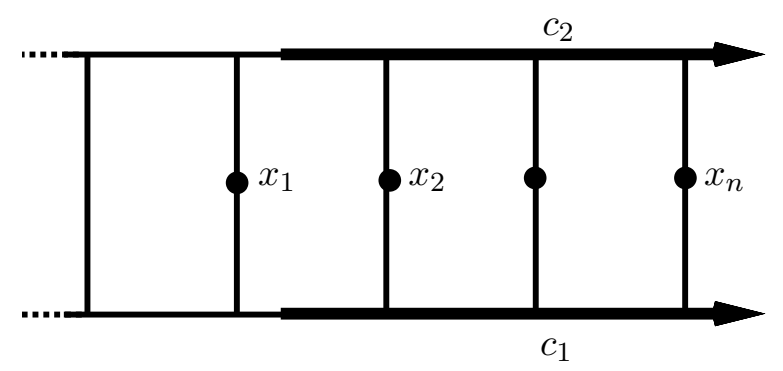

$(\mathrm{A})$

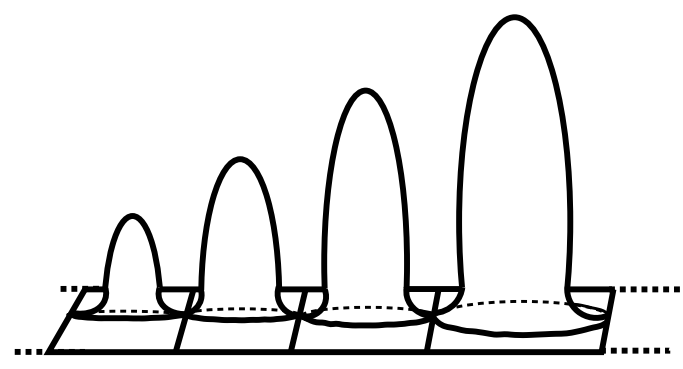

(B)

Figure 5: (A) A simple modification of Figure 4 which satisfies $\partial_{\mathcal{B}} M \subsetneq \partial_{\mathcal{G}} M$. The Busemann functions for the horizontal curves $c_{1}, c_{2}$ yield two boundary points $z_{1}, z_{2}$ in $\partial_{\mathcal{B}} M$. The sequence $\left\{x_{n}\right\}$ converges to both, $z_{1}$ and $z_{2}$, in the chr. topology, and to a different point $z$ of $\partial_{\mathcal{G}} M$ in the pointwise topology.

(B) Filling the holes in (A) by using diverging (or just big enough) "bubbles", a complete simply-connected space which behaves qualitatively as the previous one $\left(\partial_{\mathcal{B}} M \subsetneq \partial_{\mathcal{G}} M\right)$ is constructed. Positive sectional curvature is required in some regions (see Remark 4.18). 


\section{$5 \quad$ Finslerian completions}

Our aim in this section is to study previous compactifications for a (connected) smooth manifold $M$ endowed with the generalized distance $d$ associated to a Finsler metric $F$ on $M$. As in the Riemannian case, this can be done in somewhat more general spaces.

Convention 5.1 Along the present section, $(M, d)$ will denote any generalized metric space satisfying: $M$ is locally compact and contains a countable dense set, and $d$ is derived from a length space (except if specified otherwise). Recall that most of the notions of the previously studied symmetric case can be translated to the present non-symmetric case in a straightforward way; in particular, for a smooth curve, the Finslerian norm $F(\dot{c})$ must be interpreted as the dilatation (see for example [8, Appendix], and also Convention 4.1). The extended quasi-distance on the Cauchy completions $M_{C}^{ \pm}$is denoted by $d_{Q}$.

In order to translate previous constructions to a generalized metric space $(M, d)$, first we need to generalize the notion of Lipschitz function.

Definition 5.2 A function $f: M \rightarrow \mathbb{R}$ on a generalized metric space $(M, d)$ is:

- 1-Lipschitz or, simply, Lipschitz, if $f(y)-f(x) \leq d(x, y)$ for all $x, y \in M$.

- Max-Lipschitz if $|f(x)-f(y)| \leq \max \{d(x, y), d(y, x)\}$ for all $x, y \in M$.

The sets of all the Lipschitz and Max-Lipschitz functions on $(M, d)$ will be denoted $\mathcal{L}_{1}(M, d)$ and $\mathcal{L}_{1}^{\mathrm{Max}}(M, d)$, resp.

Remark 5.3 (1) In principle, the space $\mathcal{L}_{1}^{\operatorname{Max}}(M, d)$ uses $d$ in a symmetric way and seems closer to the one used in the Riemannian case. However, this space (as well as the notion of Lipschitz function for the symmetrized distance $d^{s}$, which is defined in the obvious way) has the drawback that the involved distance does not come from a length space (see [8], especially its Appendix, for a discussion and examples in the class of Randers metrics). This and other reasons of compatibility with the causal boundary justify that, in what follows, the space $\mathcal{L}_{1}(M, d)$ will be preferred. However, the space $\mathcal{L}_{1}^{\operatorname{Max}}(M, d)$ will be useful for technical purposes.

(2) The reasonings in Remark 4.3 are applicable to $\mathcal{L}_{1}^{\operatorname{Max}}(M, d)$. So, this space is metrizable and the pointwise, compact-open and uniform topologies coincide on it. Moreover, Lemma 4.4 remains true if we replace $\mathcal{L}_{1}\left(M, d^{R}\right)$ by $\mathcal{L}_{1}^{\operatorname{Max}}(M, d)$ and, then, a first Gromov-type compactification as in Theorem 4.12 would be defined by using the space $\mathcal{L}_{1}^{\operatorname{Max}}(M, d)_{*}=\mathcal{L}_{1}^{\operatorname{Max}}(M, d) / \mathbb{R}$.

Now, for $\mathcal{L}_{1}(M, d)$ :

Proposition 5.4 (1) $\mathcal{L}_{1}(M, d)$ is a closed subset of $\mathcal{L}_{1}^{\operatorname{Max}}(M, d)$.

(2) Let $\left\{f_{n}\right\}$ in $\mathcal{L}_{1}(M, d)$ with $\left\{f_{n}\left(x_{0}\right)\right\}$ converging to $k_{0} \in[-\infty, \infty]$ for some $x_{0} \in M$. If $k_{0} \in \mathbb{R}$ then it admits a pointwise convergent subsequence; otherwise, it converges uniformly on compact sets to $\pm \infty$.

(3) The uniform, compact-open and pointwise topologies are equivalent on $\mathcal{L}_{1}(M, d)$. 
Proof. For (1), let $f$ be the pointwise limit of a sequence $\left\{f_{n}\right\} \subset \mathcal{L}_{1}(M, d)$. Note that, if $f(y)>f(x)$, then $f_{n}(y)>f_{n}(x)$ for $n$ big enough, thus $f(y)-f(x)=$ $\lim _{n}\left(f_{n}(y)-f_{n}(x)\right)<d(x, y)$.

The proof of (2) follows from the facts that Lemma 4.4 is true for $\mathcal{L}_{1}^{\mathrm{Max}}(M, d)$, and $\mathcal{L}_{1}(M, d)$ is a closed subset of $\mathcal{L}_{1}^{\mathrm{Max}}(M, d)$. Item $(3)$ is proved analogously.

\subsection{Gromov completions for the non-symmetric case}

Endow $\mathcal{L}_{1}(M, d)$ with the pointwise convergence topology and $M_{C}^{ \pm}$with its natural quasi-distance $d_{Q}$ and topologies (Convention 3.27). Clearly, the maps

$$
\begin{array}{lll}
\hat{j}^{+}: M_{C}^{+} \rightarrow \mathcal{L}_{1}(M, d), & x \mapsto-d_{x}^{+}, & \text {where } d_{x}^{+}=d_{Q}(\cdot, x) \\
\hat{j}^{-}: M_{C}^{-} \rightarrow \mathcal{L}_{1}(M, d), & x \mapsto+d_{x}^{-}, & \text {where } d_{x}^{-}=d_{Q}(x, \cdot)
\end{array}
$$

define two injective functions. Up to a sign, the second one can be regarded as the first one for $d_{Q}^{\text {rev }}$. In what follows, we will consider only the map $\hat{j}^{+}$(being the case for $\hat{j}^{-}$completely analogous). The map $\hat{j}^{+}$is not automatically continuous, as happened in the Riemannian case (Prop. 4.5). However:

Lemma 5.5 The map $\hat{j}^{+}: M_{C}^{+} \rightarrow \mathcal{L}_{1}(M, d)$ is continuous if and only if $d_{Q}$ satisfies the following property:

(a4') For any sequence $\left\{x_{n}\right\} \subset M_{C}^{+}$and $x \in M_{C}^{+}$such that $\lim _{n} d_{Q}\left(x_{n}, x\right)=0$, necessarily $\lim _{n} d_{Q}\left(x, x_{n}\right)=0$, i.e. the topology generated by the backward balls is finer than the one generated by the forward balls.

Moreover, if $d_{Q}$ is a generalized distance, then $\hat{j}^{+}$is an embedding.

Proof. For the implication to the right, suppose that $\hat{j}^{+}$is continuous and consider a sequence $\left\{x_{n}\right\} \subset M_{C}^{+}$and a point $x$ in $M_{C}^{+}$such that $\lim _{n} d_{Q}\left(x_{n}, x\right)=0$. By the continuity of $\hat{j}^{+}$, necessarily $\left\{\hat{j}^{+}\left(x_{n}\right)\right\} \rightarrow \hat{j}^{+}(x)$, i.e. $\lim _{n} d_{Q}\left(\cdot, x_{n}\right)=d_{Q}(\cdot, x)$. Then, the result follows by evaluating the last expression in $x$.

For the implication to the left, suppose that condition (a4') is satisfied and take $\left\{x_{n}\right\} \subset M_{C}^{+}, x \in M_{C}^{+}$with $\left\{x_{n}\right\} \rightarrow x$, i.e. $\lim _{n} d_{Q}\left(x_{n}, x\right)=0$. Then:

$$
\begin{aligned}
d_{Q}(\cdot, x) & \leq \lim _{n}\left(d_{Q}\left(\cdot, x_{n}\right)+d_{Q}\left(x_{n}, x\right)\right) \\
& \leq \lim _{n}\left(d_{Q}(\cdot, x)+d_{Q}\left(x, x_{n}\right)+d_{Q}\left(x_{n}, x\right)\right) .
\end{aligned}
$$

Taking into account that $\lim _{n} d_{Q}\left(x_{n}, x\right)=\lim _{n} d_{Q}\left(x, x_{n}\right)=0$, the intermediate equality holds, i.e. $d_{Q}(\cdot, x)=\lim _{n} d_{Q}\left(\cdot, x_{n}\right)$, and so, $\hat{j}^{+}\left(x_{n}\right) \rightarrow \hat{j}^{+}(x)$.

For the last assertion, recall that any sequence $\left\{d_{Q}\left(\cdot, x_{n}\right)\right\}$ converging pointwise to $d_{Q}(\cdot, x)$ satisfies that $\lim _{n} d_{Q}\left(x, x_{n}\right)=d_{Q}(x, x)=0$. So, from (a4) in Definition 3.1. $\lim _{n} d_{Q}\left(x_{n}, x\right)=0$, i.e., $\left\{x_{n}\right\}$ converges to $x$.

Remark 5.6 Observe that condition (a4) in Definition 3.1 is more restrictive than (a4') here (see also Remark 3.2. . So, if $d_{Q}$ is a generalized distance then $\hat{j}^{+}$is continuous (and analogously for $\hat{j}^{-}$). 
From Lemma 5.5, and reasoning as in Proposition 4.6 and Remark 4.7 one deduces:

Proposition 5.7 Consider the natural map

$$
j^{+}: M_{C}^{+} \rightarrow \mathcal{L}_{1}(M, d)_{*}, \quad x \mapsto\left[-d_{x}^{+}\right] .
$$

Then, $\left.j^{+}\right|_{M}$ is a topological embedding. Moreover $j^{+}$is continuous if and only if $d_{Q}$ satisfies (a4'). If, in addition, $d_{Q}$ is a generalized distance and $M_{C}^{+}$is locally compact then $j^{+}$is also an embedding.

Definition 5.8 Let $(M, d)$ be the generalized metric space associated to a Finsler manifold (or any space under Convention 5.1). The forward (resp. backward) Gromov completion $M_{G}^{+}$(resp. $\left.M_{G}^{-}\right)$of $(M, d)$ is the closure of $j^{+}(M)$ (resp. $j^{-}(M)$ ) in $\mathcal{L}_{1}(M, d)_{*}$.

Then, the forward (resp. backward) Gromov boundary $\partial_{G}^{+} M$ (resp. $\left.\partial_{G}^{-} M\right)$ of $M$ is defined as $\partial_{G}^{+} M=M_{G}^{+} \backslash j^{+}(M)$ (resp. $\partial_{G}^{-} M=M_{G}^{-} \backslash j^{-}(M)$ ).

Theorem $5.9 M_{G}^{+}$and $\partial_{G}^{+} M$ are compact metrizable topological spaces.

The image of the manifold $j^{+}(M) \subset M_{G}$ is an open dense subset of $M_{G}^{+}$and, thus, the boundary $\partial_{G}^{+} M$ is also compact.

Proof. Notice that the map $i: \mathcal{L}_{1}(M, d)_{*} \rightarrow \mathcal{L}_{1}^{\operatorname{Max}}(M, d)$, which sends each class $[f]$ to its representative $f_{0}$ such that $f_{0}\left(x_{0}\right)=0$ for some fix $x_{0} \in M$, is an embedding. So, the sequential compactness follows Prop. 5.4 (2), and the result follows taking into account that $\mathcal{L}_{1}^{\operatorname{Max}}(M, d)$ is metrizable (Remark $5.3(2)$ ).

As in Theorem 4.12, the second assertion follows from standard arguments.

In analogy with the Riemannian case, the Gromov boundary (and the Gromov completion analogously) can be divided in two disjoint parts. One of them is the Cauchy-Gromov boundary, $\partial_{C G}^{+} M$, which contains all the points which are limit of some sequence of $M$ included in some bounded subset, i.e. which are contained in some (forward) ball $B^{+}(x, r)$ with $x \in M$ and $r>0$. The other part is the proper Gromov boundary, $\partial_{\mathcal{G}}^{+} M=\partial_{G}^{+} M \backslash \partial_{C G}^{+} M$. The Cauchy-Gromov boundary contains the Cauchy boundary as a point set, so we define the residual Gromov boundary, $\partial_{R G}^{+} M:=\partial_{C G}^{+} M \backslash j^{+}\left(\partial_{C}^{+} M\right)$. Consequently, the Cauchy-Gromov completion is defined as $M_{C G}^{+}=M \cup \partial_{C G}^{+} M$ and $M_{G}^{+}=M_{C G}^{+} \cup \partial_{\mathcal{G}}^{+} M$.

In order to understand the structure of $M_{C G}^{+}$, a Finslerian version of Corollary 4.13 is required. Recall however that, in the proof of that corollary, Arzela's Theorem was applied to a sequence of curves. One can prove directly a version of Arzela's Theorem for generalized distances. But this would be insufficient for our purposes here, as we will also need to include a generalization of the notion of equicontinuity 7

\footnotetext{
${ }^{7}$ As an alternative to Arzela's Theorem, the extended version of Hopf-Rinow Theorem for spaces under Convention 4.1 (see [16]) could have been used. In order to follow such an approach here, a Finslerian version of this extended Hopf-Rinow Theorem would be required. Our approach below also allows to prove this version, and makes apparent the difficulties associated to non-symmetry.
} 
Definition 5.10 Let $(M, d)$ be a generalized metric space associated to a Finsler manifold. A sequence of functions $\left\{f_{n}\right\}, f_{n}:[a, b] \rightarrow M$, is oriented-equicontinuous if, for any $\epsilon>0$, there exists $\delta>0$ such that, for any $t_{1}, t_{2} \in[a, b]$ with $0 \leq t_{2}-t_{1}<$ $\delta$, then $d\left(f_{n}\left(t_{1}\right), f_{n}\left(t_{2}\right)\right)<\epsilon$ for all $n$. If the requirement $0 \leq t_{2}-t_{1}<\delta$ is weakened into $0 \leq\left|t_{2}-t_{1}\right|<\delta$, then $\left\{f_{n}\right\}$ is equicontinuous.

Remark 5.11 (1) If the sequence $\left\{f_{n}\right\}$ is oriented-equicontinuous, then each $f_{n}$ is continuous. In fact, fix $c \in[a, b]$ and consider a sequence $\left\{t_{m}\right\} \rightarrow c$. With no loss of generality, consider the cases: (i) $t_{m} \leq c$ and (ii) $t_{m} \geq c$. For (i), as $f_{n}$ belongs to an oriented-equicontinuous sequence, the definition yields directly $\left\{d\left(f_{n}\left(t_{m}\right), f_{n}(c)\right)\right\}_{m} \rightarrow 0$. For (ii), the oriented-equicontinuity ensures that $\left\{d\left(f_{n}(c), f_{n}\left(t_{m}\right)\right)\right\}_{m} \rightarrow 0$ and then, by using that $d$ is a generalized distance, we deduce $\left\{d\left(f_{n}\left(t_{m}\right), f_{n}(c)\right)\right\}_{m} \rightarrow 0$ from condition (a4) in Definition 3.1. as required.

(2) Oriented-equicontinuity does not imply equicontinuity. In fact, one can easily construct an example of this situation by endowing the space in Figure 3 with a Finslerian metric such that the vertical segments have unitary lengths when they are parameterized by a curve from down to up, and diverging lengths when they are parameterized from up to down.

The following result is the (local) version of Arzela's Theorem for equicontinuity in generalized metric spaces, which will be refined later for oriented-equicontinuity (Theorem 5.15).

Theorem 5.12 Let $(M, d)$ be a generalized metric space and $\left\{f_{n}\right\}, f_{n}:[a, b] \rightarrow M$, a sequence of equicontinuous functions. Assume that $x \in M$ is an accumulation point of $\left\{f_{n}(c)\right\}$, with $c \in[a, b]$, admitting a compact neighborhood $K$. Then, there exist $r>0$, a subsequence $\left\{f_{n_{k}}\right\} \subset\left\{f_{n}\right\}$ and a continuous function $f:[c-r, c+$ $r] \cap[a, b] \rightarrow M$ such that $\left\{f_{n_{k}}(t)\right\}_{k} \rightarrow f(t)$ for each $t \in[c-r, c+r] \cap[a, b]$.

Proof. It is carried out by standard arguments, which we include for the sake of completenes $\AA^{8}$. By the equicontinuity, and the facts that $x$ is an accumulation point and $d$ a generalized distance, there exists $r>0$ such that $f_{n}([c-r, c+r] \cap[a, b]) \subset K$ for all $n$ big enough. Let $A=\left\{t_{m}\right\}_{m=0}^{\infty} \subset[c-r, c+r] \cap[a, b]$ be a countable dense subset, and consider the sequence of sequences $\left\{f_{n}\left(t_{m}\right)\right\}_{n} \subset K$. A standard diagonal argument (see the proof of Lemma 4.4 ensures the existence of a subsequence $\left\{f_{n_{k}}\right\}_{k}$ such that, for each $m,\left\{f_{n_{k}}\left(t_{m}\right)\right\}_{k}$ converges to some point, denoted $f\left(t_{m}\right)$.

Now, we are in conditions to prove that $\left\{f_{n_{k}}(t)\right\}$ is convergent for each $t \in$ $[c-r, c+r] \cap[a, b]$. In fact, as the sequence $\left\{f_{n_{k}}(t)\right\}_{k}$ is contained in the compact $K$, it suffices to show that it is a Cauchy sequence. Take $\epsilon>0$, from the equicontinuity of $\left\{f_{n_{k}}\right\}$ and the density of $A$, there exists $t_{m} \in A$ such that $d\left(f_{n_{k}}\left(t_{m}\right), f_{n_{k}}(t)\right), d\left(f_{n_{k}}(t), f_{n_{k}}\left(t_{m}\right)\right)<\epsilon / 3$ for all $k$. On the other hand, as $\left\{f_{n_{k}}\left(t_{m}\right)\right\}$ is a Cauchy sequence, there exists $k_{0}$ such that $d\left(f_{n_{k_{1}}}\left(t_{m}\right), f_{n_{k_{2}}}\left(t_{m}\right)\right)<\epsilon / 3$ for $k_{2}>k_{1} \geq k_{0}$ and, so:

$$
\begin{aligned}
d\left(f_{n_{k_{1}}}(t), f_{n_{k_{2}}}(t)\right) \leq & d\left(f_{n_{k_{1}}}(t), f_{n_{k_{1}}}\left(t_{m}\right)\right)+d\left(f_{n_{k_{1}}}\left(t_{m}\right), f_{n_{k_{2}}}\left(t_{m}\right)\right) \\
& +d\left(f_{n_{k_{2}}}\left(t_{m}\right), f_{n_{k_{2}}}(t)\right)<\frac{\epsilon}{3}+\frac{\epsilon}{3}+\frac{\epsilon}{3}=\epsilon,
\end{aligned}
$$

\footnotetext{
${ }^{8}$ Recall that here not all the assumptions in Convention 5.1 are required.
} 
as required. Let $f(t)$ be the limit point of the sequence $\left\{f_{n_{k}}(t)\right\}_{k}$. The continuity of the so obtained $f$ follows from the equicontinuity of the sequence $\left\{f_{n_{k}}\right\}$.

In order to deal with oriented-equicontinuity, let us consider the following result.

Lemma 5.13 Let $(M, d)$ be a generalized metric space associated to a Finsler manifold, and $\left\{f_{n}\right\}, f_{n}:[a, b] \rightarrow M$, a sequence of oriented-equicontinuous functions satisfying $f_{n}([a, b]) \subset K$, with $K$ compact. Then, the sequence $\left\{f_{n}\right\}$ is equicontinuous.

Proof. Assume by contradiction that $\left\{f_{n}\right\}$ is not equicontinuous. Then, there exist $\epsilon>0$, two sequences $\left\{t_{m}^{1}\right\},\left\{t_{m}^{2}\right\} \subset[a, b]$ and a subsequence $\left\{f_{n_{m}}\right\}_{m}$ such that $0<t_{m}^{2}-t_{m}^{1} \searrow 0$ and

$$
d\left(f_{n_{m}}\left(t_{m}^{2}\right), f_{n_{m}}\left(t_{m}^{1}\right)\right)>\epsilon \text { for all } m .
$$

Even more, since $\left\{f_{n}\right\}$ is oriented-equicontinuous, we can also assume that

$$
d\left(f_{n_{m}}\left(t_{m}^{1}\right), f_{n_{m}}\left(t_{m}^{2}\right)\right)<1 / m \text { for all } m .
$$

As $\left\{f_{n_{m}}\left(t_{m}^{i}\right)\right\}_{m}, i=1,2$, is contained in the compact $K$, up to a subsequence, we can assume that $\left\{f_{n_{m}}\left(t_{m}^{i}\right)\right\}_{m} \rightarrow x^{i}$ for some $x^{i} \in K, i=1,2$. From (5.2), $d\left(x^{2}, x^{1}\right) \geq \epsilon$, but from $(5.3), d\left(x^{1}, x^{2}\right)=0$, in contradiction to the fact that $d$ is a generalized distance.

Remark 5.14 As a consequence, if $(M, d)$ is locally compact and $\left\{f_{n}\right\}_{n}$ converges pointwise to a continuous function $f$, then the family $\left\{f_{n}\right\}_{n \geq n_{0}}$ is equicontinuous for some $n_{0}$. In fact, the compactness of $f([a, b])$ implies the existence of a compact neighborhood $K$ of $f([a, b])$, and the inclusion $f_{n}([a, b]) \subset K$ follows from the uniform convergence of $\left\{f_{n}\right\}$ to $f$.

The required version of Arzela's Theorem is the following:

Theorem 5.15 Let $(M, d)$ be a generalized metric space and $\left\{f_{n}\right\}, f_{n}:[a, b] \rightarrow$ $M$, a sequence of oriented-equicontinuous functions. Suppose that $\left\{f_{n}(a)\right\}$ has an accumulation point and $(M, d)$ is locally compact and complete. Then, there exist a continuous function $f:[a, b] \rightarrow M$ and an equicontinuous subsequence $\left\{f_{n_{k}}\right\} \subset\left\{f_{n}\right\}$ such that $\left\{f_{n_{k}}(t)\right\} \rightarrow f(t)$ for each $t \in[a, b]$.

Proof. By Remark 5.14, it suffices to prove the existence of a continuous function $f:[a, b] \rightarrow M$ and a subsequence $\left\{f_{n_{k}}\right\} \subset\left\{f_{n}\right\}$ such that $\left\{f_{n_{k}}(t)\right\} \rightarrow f(t)$ for each $t \in[a, b]$. To this aim, consider the set

$$
B=\left\{r \in(a, b]: \begin{array}{l}
\exists \text { subsequence of }\left\{f_{n}\right\} \text { which converges (pointwise) } \\
\text { to a continuous function } f:[a, r] \rightarrow M
\end{array}\right\} .
$$

Reasoning as in Theorem 5.12 there exists $r>0$ such that $f_{n}([a, a+r]) \subset K$ for all $n$ and for some compact set $K$. From Lemma 5.13 the family $\left\{f_{n}\right\}$ is equicontinuous in $[a, a+r]$, and from Theorem 5.12 necessarily $B \neq \emptyset$. Let $r_{0} \leq b$ 
be the supremum of $B$. By a canonical diagonal argument, there exists a continuous function $f:\left[a, r_{0}\right) \rightarrow M$ which is the pointwise limit of a partial $\left\{f_{n_{k}}\right\}$ of $\left\{f_{n}\right\}$. Then, define recursively a sequence $\left\{s_{m}\right\}$ in the following way:

$$
s_{m} \geq s_{m-1}, \quad 0<r_{0}-s_{m}<\delta_{m}<\min \left\{\delta_{m-1}, 1 / m\right\},
$$

where $\delta_{0}=1$ and $\delta_{m}$ is obtained from the oriented-equicontinuity of $\left\{f_{n_{k}}\right\}$ applied to $\epsilon=1 / m$. The sequence $\left\{s_{m}\right\}$ satisfies that $\left\{s_{m}\right\} \nearrow r_{0}$ and $\left\{f\left(s_{m}\right)\right\}_{m}$ is a Cauchy sequence. From the completeness of $M,\left\{f\left(s_{m}\right)\right\}_{m}$ converges to some point $f\left(r_{0}\right)$. As $M$ is locally compact, there exists $r>0$ such that $B^{+}\left(f\left(r_{0}\right), r\right) \subset K^{\prime}$ with $K^{\prime}$ compact. Since $f\left(s_{m}\right) \rightarrow f\left(r_{0}\right)$ and $d$ is a generalized distance, there exists $m$ such that $d\left(f\left(r_{0}\right), f\left(s_{m}\right)\right)<r / 3$ and $1 / m<r / 3$. Moreover, as $f_{n_{k}}\left(s_{m}\right) \rightarrow f\left(s_{m}\right)$, for $n_{k}$ big enough $d\left(f\left(s_{m}\right), f_{n_{k}}\left(s_{m}\right)\right)<r / 3$. Therefore, we have that, for all $s \in$ $\left[s_{m}, s_{m}+\delta_{m}\right]$ and $n_{k}$ big enough,

$$
\begin{aligned}
d\left(f\left(r_{0}\right), f_{n_{k}}(s)\right) & \leq d\left(f\left(r_{0}\right), f\left(s_{m}\right)\right)+d\left(f\left(s_{m}\right), f_{n_{k}}\left(s_{m}\right)\right)+d\left(f_{n_{k}}\left(s_{m}\right), f_{n_{k}}(s)\right) \\
& <\frac{r}{3}+\frac{r}{3}+\frac{r}{3}=r .
\end{aligned}
$$

So, for $n_{k}$ big enough, $f_{n_{k}}\left(\left[s_{m}, s_{m}+\delta_{m}\right]\right) \subset K^{\prime}$ and then, by Lemma 5.13 , the family $f_{n_{k}}$ is equicontinuous in $\left[s_{m}, s_{m}+\delta_{m}\right]$. Moreover, as $r_{0}-s_{m}<\delta_{m}$, necessarily $r_{0} \in$ $\left[s_{m}, s_{m}+\delta_{m}\right]$. Therefore, from the equicontinuity and the fact that $f\left(s_{m}\right) \rightarrow f\left(r_{0}\right)$, we deduce that $f_{n_{k}}\left(r_{0}\right) \rightarrow f\left(r_{0}\right)$. Finally, by Theorem $5.12, r_{0}$ must be a maximum, and thus, $r_{0}=b$.

Now we are in conditions to prove:

Corollary 5.16 The Cauchy-Gromov completion $M_{C G}^{+}=M \cup \partial_{C G}^{+} M$ satisfies:

(1) (Openness of $M$ ). The boundary $\partial_{C G}^{+} M$ is closed in $M_{C G}^{+}$.

(2) (Heine-Borel). The closure in $M_{C G}^{+}$of any bounded set in $M$ is compact.

(3) (Consistency with the Cauchy boundary). If $M_{C}^{+}$is locally compact and $d$ is a generalized distance, then $j^{+}: M_{C}^{+} \rightarrow M_{G}^{+}$is an embedding and $M_{C}^{+} \equiv M_{C G}^{+}$, i.e. $\partial_{R G}^{+} M=\emptyset$.

Proof. The first assertion is straightforward from Theorem 5.9. The second assertion follows from the compact character of $M_{G}^{+}$and the definition of $M_{C G}^{+}$. For the first assertion in (3), as $d$ is a generalized distance, condition (a4') holds, and thus, $j^{+}$ is continuous. The continuity of the inverse of $j^{+}$follows reasoning as in Remark 4.7. For the last assertion, in analogy to the Riemannian case, it is sufficient to show that $\overline{B^{+}\left(x, r ; M_{C}^{+}\right)}, x \in M_{C}^{+}, r>0$, are compact. Consider a sequence $\left\{x_{n}\right\}$ in $B^{+}\left(x, r ; M_{C}^{+}\right)$and define a sequence of curves $\left\{\gamma_{n}\right\}, \gamma_{n}:[0,1] \rightarrow M$ joining $x$ and $x_{n}$, such that: each $\gamma_{n}$ is contained in $M$, it is parametrized with constant speed and length $\left(\gamma_{n}\right)<2 r$. Reasoning as in the Riemannian case (see footnote 6), the family of curves is oriented-equicontinuous. So, taking into account that $M_{C}^{+}$ is locally compact and complete, and $x$ is an accumulation point of $\left\{\gamma_{n}\right\}$, Theorem 5.15 ensures the existence of a curve $\gamma_{\infty}$ which is the pointwise limit of a subsequence of $\left\{\gamma_{n}\right\}$, and so the required limit is $\gamma_{\infty}(1)$. 


\subsection{Busemann completions}

Next, we are going to develop the Busemann completion $M_{B}^{+}$for any Finsler manifold $(M, F)$. First, in Subsection 5.2.1, we will deal with its definition as a point set by making definitions analogous to the Riemannian ones. The topology is developed in the following three subsections. As always in the non-symmetric case, a second completion $M_{B}^{-}$is possible, and some non-trivial conventions about it will be stressed at Subsection 5.2.5.

\subsubsection{Busemann completion as a point set}

Let $(M, d)$ be a generalized metric space associated to a Finsler manifold $(M, F)$ or any space under Convention 5.1. We will introduce the analogous objects to those ones in Section 4.2 for the Riemannian case.

Let $C^{+}(M)$ be the set of curves $c:[\alpha,+\Omega) \rightarrow M, \Omega \leq \infty$, such that $F(\dot{c})<1$ (recall Remark 4.17). For $c \in C^{+}(M)$ the associated (forward) Busemann function $b_{c}^{+}: M \rightarrow(-\infty, \infty]$ is:

$$
b_{c}^{+}(\cdot)=\lim _{s \rightarrow \Omega}(s-d(\cdot, c(s))
$$

which is well-defined as

$$
s \mapsto s-d(x, c(s)) \text { is increasing for any } x \in M
$$

(extend Lemma 4.14). Following steps analogous to those in Proposition 4.15, first, we have that if a Busemann function is not identically infinity then it is finite-valued everywhere, and we denote:

$$
B^{+}(M):=\left\{b_{c}^{+}<\infty: c \in C^{+}(M)\right\}
$$

Second, we also have

$$
B^{+}(M) \subset \mathcal{L}_{1}(M, d), \quad \text { and so, } \quad b_{c}^{+} \in B^{+}(M) \Rightarrow\left[b_{c}^{+}\right] \in M_{G}^{+} .
$$

Moreover, when $\Omega<\infty$ there exists some $\bar{x} \in M_{C}^{+}$such that $b_{c}^{+}(x)=\Omega-d(x, \bar{x})$ for all $x \in M$ (here, $d$ is extended to the elements of $M_{C}^{+}$according to Prop. 3.32, and we will write

$$
b_{c}^{+}=d_{p}^{+}:=\Omega-d_{\bar{x}}^{+},
$$

where $d_{\bar{x}}^{+}(x):=d(x, \bar{x})$ for all $x \in M$ and $p=(\Omega, \bar{x}) \in \mathbb{R} \times M_{C}^{+}$(recall that the domain of $d_{\bar{x}}^{+}$can be also extended to any point of $\left.M_{C}^{+}\right)$. If, otherwise, $\Omega=\infty$, then $b_{c}^{+}$is called a properly Busemann function and we write:

$$
\mathcal{B}^{+}(M):=\left\{b_{c}^{+}<\infty: c \in C^{+}(M), \Omega=\infty\right\} .
$$

Definition 5.17 As a point set, the (forward) Busemann completion of $(M, d)$ is the quotient $M_{B}^{+}:=B^{+}(M) / \mathbb{R} \subset M_{G}^{+}$, the (forward) Busemann boundary is $\partial_{B}^{+} M:=M_{B}^{+} \backslash M\left(\subset \partial_{G}^{+} M\right)$, and the (forward) properly Busemann boundary is $\partial_{\mathcal{B}}^{+} M:=\mathcal{B}^{+}(M) / \mathbb{R}$.

From previous discussion, clearly, $\partial_{B}^{+} M=j^{+}\left(\partial_{C}^{+} M\right) \cup \partial_{\mathcal{B}}^{+} M$. 


\subsubsection{Chronological topology on $M_{B}^{+}$}

The chronological topology on $B^{+}(M)$ is introduced defining the closed sets by means of a limit operator $\hat{L}$. Such an operator has its origins in an operator introduced by Flores and Harris ([1]; see also [20, 19]) in order to study the future causal boundary of a static spacetime, and it has been developed further, including some consequences for the Riemannian case (see [1, 10, 1, 21, 30, 12]). Here, we introduce directly the chronological topology for the Busemann completion of a Finsler manifold, $M_{B}^{+}$. The definition is somewhat involved, and its motivation will appear more clearly in stationary spacetimes. However, one can appreciate its nice properties and its relation with Gromov topology — even in Finsler manifolds which are not involved in constructions associated to boundaries of spacetimes.

The limit operator $\hat{L}$ will allow to define the closed sets for the topology in $B^{+}(M)$. Let $\sigma=\left\{f_{n}\right\} \subset B^{+}(M)$, the subset $\hat{L}(\sigma) \subset B^{+}(M)$ is defined as:

$$
f \in \hat{L}(\sigma) \Longleftrightarrow\left\{\begin{array}{l}
(a) f \leq \liminf _{n} f_{n} \text { and } \\
(b) \forall g \in B^{+}(M) \text { with } f \leq g \leq \limsup _{n} f_{n} \text {, it is } g=f .
\end{array}\right.
$$

Definition 5.18 The (forward) chronological topology (chr. topology for short) on $B^{+}(M)$ is the one such that $C \subset B^{+}(M)$ is closed if and only if $\hat{L}(\sigma) \subset C$ for any sequence $\sigma=\left\{f_{n}\right\} \subset C$,

The induced quotient topology on the Busemann completion $M_{B}^{+}=B^{+}(M) / \mathbb{R}$ will be also called Busemann or chr. topology on $M_{B}^{+}$.

The Busemann completion $M_{B}^{+}$will be always regarded as a topological space endowed with the chr. topology, except if otherwise is explicitly said.

Remark 5.19 It is easy to check that this notion of closed set is consistent and, moreover, if $f \in \hat{L}(\sigma)$ then $\sigma$ converges to $f$. In fact, this can be seen from the following more general viewpoint (see [12, Appendix 3.6] for details; recall also Remark 2.3(1) above).

A limit operator $L$ on a set $X$ is any map $L: \mathcal{S}(X) \rightarrow \mathcal{P}(X)$ (where $\mathcal{S}(X)$ is the set of all the sequences in $X$ and $\mathcal{P}(X)$ is the set of parts of $X$ ) such that if $\bar{\sigma}$ is a subsequence of some $\sigma \in S(X)$ then $L(\sigma) \subset L(\bar{\sigma})$. This property ensures that an associated topology $\tau_{L}$ is defined on $X$ just declaring that $C \subset X$ is closed if and only if $L(\sigma) \subset C$ for any sequence $\sigma$ in $C$. For this topology, $x \in L(\sigma)$ implies that $\sigma \rightarrow x$. The converse does not hold in general and, when it holds, $L$ (and, then, $\tau_{L}$ ) is called of first order.

Such a topological space $\left(X, \tau_{L}\right)$ is a sequential space, i.e., it satisfies that any sequentially closed subset (which is a subset $S \subset X$ such that if a sequence in $S$ converges to a point $x \in X$ then $x \in S$ ) is closed -recall that the converse always holds. In the class of sequential spaces (which includes the class of first countable ones) the continuity of a function can be characterized as sequential continuity, i.e., in terms of the preservation of converging sequences, as in the standard case of metric spaces. This fact will be used frequently for maps between spaces such as $M_{B}^{+}, B^{+}(M), M_{G}^{+}$or $M_{C}^{+}$, as all these spaces are sequential (the last two ones 
because they are metrizable, and $B^{+}(M), M_{B}^{+}$by construction, see also Proposition 5.23 .

Finally, consider the constant sequence $\hat{x}=\{x\}_{n}$, for each $x \in X$. Clearly, if $L(\hat{x})=\{x\}$ for all $x \in X$, then the points in $X$ are closed for $\tau_{L}$, i.e. $\tau_{L}$ is $T_{1}$ (conversely, if $\tau_{L}$ is $T_{1}$ then $L(\hat{x}) \subset\{x\}$ ). In particular, the chr. topology is always $T_{1}$.

An example of result (to be used later) which is valid for the chr. topology as well as any topology constructed from a limit operator $L$, is the following. Recall that it is applicable to the limit operator $\hat{L}$ for the chr. topology in $M_{B}^{+}$.

Proposition 5.20 Let $X$ be a set endowed with a topology associated to a limit operator L. Assume that $L(\hat{x})=\{x\}$ for any $x \in X$, where $\hat{x}=\{x\}_{n}$. Given a sequence $\sigma \subset X$, if $L(\bar{\sigma})=\{x\}$ for any subsequence $\bar{\sigma} \subset \sigma$ then $\sigma$ only converges to $x$.

Proof. From Remark 5.19, $x$ is a limit of $\sigma$ and we will prove that it is unique. Assume by contradiction that $x^{\prime} \neq x$ is also limit of $\sigma$. In particular, $x, x^{\prime}$ are limits of any subsequence $\bar{\sigma}$ of $\sigma$. Notice that $\bar{\sigma} \cup\{x\}$ is closed. In fact, if $\tau$ is any sequence constructed from the elements in $\bar{\sigma} \cup\{x\}$ then some subsequence $\bar{\tau}$ of $\tau$ must be either a subsequence of $\bar{\sigma}$ or constantly equal to some element of $\bar{\sigma} \cup\{x\}$. In both cases, our assumptions imply $L(\bar{\tau}) \subset \bar{\sigma} \cup\{x\}$, and then $L(\tau) \subset \bar{\sigma} \cup\{x\}$ as required. As a consequence, $x^{\prime} \in \bar{\sigma}$ for any subsequence $\bar{\sigma} \subset \sigma$. This means that $\sigma$ is constantly equal to $x^{\prime}$ up to a finite number of terms, in contradiction to $L(\sigma)=\{x\}$.

The definition of the chr. topology makes necessary to study properties of convergent sequences.

Lemma 5.21 (i) For $p_{i}=\left(t_{i}, x_{i}\right) \in \mathbb{R} \times M, i=1,2$, the inequality $d_{p_{1}}^{+}<d_{p_{2}}^{+}$(i.e. $d_{p_{1}}^{+}(x)<d_{p_{2}}^{+}(x)$ for all $\left.x \in M\right)$ holds if and only if $d\left(x_{1}, x_{2}\right)<t_{2}-t_{1}$.

(ii) For any $f \in B^{+}(M)$ there exists a sequence $\left\{p_{n}\right\} \subset \mathbb{R} \times M$ such that $d_{p_{n}}^{+}<d_{p_{n+1}}^{+}$and $f=\sup _{n} d_{p_{n}}^{+}$. Moreover, if $D \subset \mathbb{R} \times M$ is a dense subset, $\left\{p_{n}\right\}$ can be chosen included in $D$.

(iii) Let $g \in \mathcal{L}_{1}(M, d)$ and $p_{0}=\left(t_{0}, x_{0}\right) \in \mathbb{R} \times M$ such that $t_{0}<g\left(x_{0}\right)$. Then, $d_{p_{0}}^{+}<g$.

Proof. (i) The implication to the right follows by evaluating the inequality $d_{p_{1}}^{+}<d_{p_{2}}^{+}$ at $x_{1}$. For the implication to the left, just use that, from the triangle inequality, $d_{x_{2}}^{+}(y)-d_{x_{1}}^{+}(y) \leq d\left(x_{1}, x_{2}\right)$ for all $y$.

(ii) Let $f=b_{c}^{+}$for $c:[\alpha, \Omega) \rightarrow M$. Any sequence $\left\{p_{n}=c\left(t_{n}\right)\right\}$ with $\left\{t_{n}\right\} \nearrow \Omega$ fulfills the required properties. For the last assertion, recall that each $W_{n}:=\{p \in$ $\left.\mathbb{R} \times M: d_{p_{n}}^{+}<d_{p}^{+}<d_{p_{n+1}}^{+}\right\}$is open and non-empty (use (i)), and replace the original $p_{n}$ by any point in $D \cap W_{n}$.

(iii) Notice that $d_{p_{0}}^{+}(x)<g\left(x_{0}\right)-d\left(x, x_{0}\right) \leq g(x)$, the last inequality from the Lipschitz condition.

The following result exhibits some natural open subsets. 
Proposition 5.22 For each $f \in B^{+}(M)$ the subset

$$
I^{+}(f):=\left\{h \in B^{+}(M): \exists p \in \mathbb{R} \times M \text { such that } f \leq d_{p}^{+}<h\right\} \subset B^{+}(M)
$$

is open for the chr. topology.

Proof. In order to prove that $B^{+}(M) \backslash I^{+}(f)$ is closed, it is enough to check that, for any sequence $\sigma=\left\{g_{n}\right\} \subset B^{+}(M)$ and function $h \in I^{+}(f)$ such that $h \in \hat{L}(\sigma)$, the sequence $\sigma$ is contained in $I^{+}(f)$, up to a finite subset. Let $p_{0}=\left(t_{0}, x_{0}\right) \in \mathbb{R} \times M$ such that $f \leq d_{p_{0}}^{+}<h$, recall that $t_{0}=d_{p_{0}}^{+}\left(x_{0}\right)<h\left(x_{0}\right)$ and, as $h \leq \liminf g_{n}$, $d_{p_{0}}^{+}\left(x_{0}\right)<g_{n}\left(x_{0}\right)$ for $n$ big enough. So, from Lemma 5.21 (iii) $d_{p_{0}}^{+}<g_{n}$ and $g_{n} \in I^{+}(f)$ for $n$ large enough.

In the particular case of $f=d_{p_{0}}^{+}$, for $p_{0} \in \mathbb{R} \times M$, the notation will be replaced by $I^{+}\left(p_{0}, B^{+}(M)\right):=I^{+}\left(d_{p_{0}}^{+}\right)$. Alternatively, by using the Lipschitz property of $h$ in (5.8):

$$
I^{+}\left(p_{0}, B^{+}(M)\right):=\left\{h \in B^{+}(M): t_{0}<h\left(x_{0}\right)\right\} \quad \text { for any } p_{0}=\left(t_{0}, x_{0}\right) \in \mathbb{R} \times M .
$$

The notation suggests that $I^{+}\left(p_{0}, B^{+}(M)\right)$ is related with the chronological future of $p_{0}$ in some spacetime, as shown explicitly in Section 6 .

The natural topological properties of $B^{+}(M)$ are transmitted to $M_{B}^{+}$. In fact, as the maps $T_{k}: B^{+}(M) \rightarrow B^{+}(M), f \mapsto f+k$ are homeomorphisms for all $k \in \mathbb{R}$, we have:

Proposition 5.23 The natural projection $\Pi: B^{+}(M) \rightarrow M_{B}^{+}=B^{+}(M) / \mathbb{R}$, is an open map.

\subsubsection{The inclusion of $M_{C}^{+}$in $M_{B}^{+}$from the topological viewpoint}

In order to obtain a true completion, the original space $M$ has to be embedded as a dense subset in $M_{B}^{+}$in a natural way. In fact, we know that $j^{+}: M_{C}^{+} \rightarrow M_{G}^{+}$is injective, and $j^{+}\left(M_{C}^{+}\right) \subset M_{B}^{+}$. Denote

$$
j_{B}^{+}: M_{C}^{+} \rightarrow M_{B}^{+}
$$

the map obtained by restricting the codomain of $j^{+}$to $M_{B}^{+}$(which is regarded now as a topological space with the chr. topology). We will see that $\left.j_{B}^{+}\right|_{M}$ yields the required embedding and, as in the case of the embedding in $M_{G}^{+}$, the situation is subtler for $j_{B}^{+}$.

Proposition 5.24 Consider a sequence $\left\{b_{c_{n}}^{+}\right\} \subset B^{+}(M)$ and $p=(\Omega, x) \in \mathbb{R} \times M$. If $d_{p}^{+}=\Omega-d_{x}^{+}\left(\in B^{+}(M)\right)$ belongs to $\hat{L}\left(\left\{b_{c_{n}}^{+}\right\}\right)$, then $\left\{b_{c_{n}}^{+}\right\}$converges pointwise to $d_{p}^{+}$. Moreover, for $n$ big enough, $b_{c_{n}}^{+}=\Omega_{n}-d_{x_{n}}^{+}$with $\Omega_{n} \in \mathbb{R}$ and $x_{n} \in M$ satisfying $\Omega_{n} \rightarrow \Omega$ and $x_{n} \rightarrow x$.

If $d_{Q}$ is a generalized distance and $M_{C}^{+}$(necessarily equal to $M_{C}^{-}$and $M_{C}^{s}$, Prop. 3.28) is locally compact, then the same conclusion holds for any $p=(\Omega, x) \in$ $\mathbb{R} \times M_{C}^{+}$. 
Proof. Consider $c_{n}:\left[\alpha_{n}, \Omega_{n}\right) \rightarrow M$ and suppose by contradiction that $\Omega-d_{x}^{+} \in$ $L\left(\left\{b_{c_{n}}^{+}\right\}\right)$but $\left\{b_{c_{n}}^{+}\right\}$does not converge pointwise to $\Omega-d_{x}^{+}$. By the chronological convergence, one has $\Omega \leq \liminf b_{c_{n}}^{+}(x)$. So, taking a subsequence $\left\{\Omega_{k_{n}}\right\}_{n}$ if necessary, and re-naming $\Omega_{k_{n}} \equiv \Omega_{n}$ for all $n$, we can assume $\Omega_{n} \geq \Omega-1 / n$. Notice also that there exists some $\epsilon>0$ such that:

$$
\Omega_{n}>\Omega+\epsilon, \text { up to a subsequence. }
$$

In fact, otherwise $\Omega_{n} \rightarrow \Omega$ and for $n$ large enough, $b_{c_{n}}^{+}=\Omega_{n}-d_{x_{n}}^{+}$for some $x_{n} \in M_{C}^{+}$ (Prop. 4.15. Since $\Omega \leq \liminf _{n} b_{c_{n}}^{+}(x)$, for each $m \in \mathbb{N}$ there exists some $m_{0}$ such that $\Omega-1 / m \leq b_{c_{n}}^{+}(x)=\Omega_{n}-d\left(x, x_{n}\right)$ for all $n \geq n_{0} \geq m$, that is:

$$
0 \leq d\left(x, x_{n}\right) \leq\left(\Omega_{n}-\Omega\right)+\frac{1}{m} .
$$

Thus, $d\left(x, x_{n}\right) \rightarrow 0$, which joined to $\Omega_{n} \rightarrow \Omega$, implies that $\left\{b_{c_{n}}^{+}\right\}$converges pointwise to $\Omega-d_{x}^{+}$, a contradiction.

Next, assuming that (5.11) holds, we are going to obtain a contradiction with the maximality of $\Omega-d_{x}^{+}$as a $L$-limit according to (b) in (5.7). The local compactness of $M$ allows to take a compact neighborhood $U$ of $x$ and define the following subsets of Busemann functions, which are also compact with the pointwise topology:

$$
\begin{gathered}
C:=\left\{t-d_{y}^{+} \in B^{+}(M): t \in\left[\Omega-\frac{\epsilon}{2}, \Omega+\frac{\epsilon}{2}\right], y \in U\right\} \\
\partial C:=\left\{t-d_{y}^{+} \in C: \text { either } t=\Omega-\frac{\epsilon}{2}, \Omega+\frac{\epsilon}{2} \text { or } y \in \partial U\right\} .
\end{gathered}
$$

For the proof, we will need the following claim which will be proved later:

Claim: For any $s \in\left[\Omega-\frac{\epsilon}{2}, \Omega\right]$ and $z \in U$ such that $s<\Omega-d(z, x)$, there exist $t^{z} \in[\Omega-\epsilon / 2, \Omega+\epsilon / 2], y^{z} \in U$ and a subsequence $\left\{b_{c_{n_{k}}}^{+}\right\}$such that: (i) $t^{z}-d_{y^{z}}^{+} \in \partial C$, (ii) $s \leq t^{z}-d_{y^{z}}^{+}(z)$ and (iii) $\liminf b_{c_{n_{k}}}^{+} \geq t^{z}-d_{y^{z}}^{+}$.

Let us apply the claim to each $s_{m}$ of a sequence $\left\{s_{m}\right\} \subset\left[\Omega-\frac{\epsilon}{2}, \Omega\right]$ with $s_{m} \nearrow \Omega$ and $z=x$. We find some $t^{m} \in\left[\Omega-\frac{\epsilon}{2}, \Omega+\frac{\epsilon}{2}\right], y^{m} \in U$ and a subsequence $\left\{b_{c_{n}}^{+[m]}\right\}_{n}$ of $\left\{b_{c_{n}}^{+}\right\}$satisfying (i), (ii) and (iii). Making each $\left\{b_{c_{n}}^{+[m+1]}\right\}_{n}$ be a subsequence of $\left\{b_{c_{n}}^{+[m]}\right\}_{n}$, by a diagonal argument we can suppose that the same subsequence $\left\{b_{c_{n_{k}}}^{+}\right\}$is valid for all $m$. Up to a subsequence, $\left\{t^{m}\right\}$ and $\left\{y^{m}\right\}$ converge to some $t^{0} \in\left[\Omega-\frac{\epsilon}{2}, \Omega+\frac{\epsilon}{2}\right], y^{0} \in U$ and, so,

$$
\left\{t^{n}-d_{y^{n}}^{+}\right\} \quad \text { converges pointwise to } t^{0}-d_{y^{0}}^{+} \in \partial C .
$$

By statements (ii) and (iii) of the claim, the function $t^{0}-d_{y^{0}}^{+}$also satisfies $\Omega \leq$ $t^{0}-d_{y^{0}}^{+}(x)$ and $\liminf b_{c_{n_{k}}}^{+} \geq t^{0}-d_{y^{0}}^{+}$. The first inequality (plus the triangle one) yields $t^{0}-d_{y^{0}}^{+} \geq \Omega-d_{x}^{+}$and, as $t^{0}-d_{y^{0}}^{+} \in \partial C$, the inequality is strict at some point. Thus, $t^{0}-d_{y^{0}}^{+}$contradicts the maximality of $\Omega-d_{x}^{+}$in $\lim \sup b_{c_{n}}^{+}$, as required.

Proof of the claim. As liminf $b_{c_{n}}^{+} \geq \Omega-d_{x}^{+}$, and so, $s<\Omega-d_{x}^{+}(z) \leq \liminf b_{c_{n}}^{+}(z)$, there exists $t_{n} \in\left(\Omega+\epsilon, \Omega_{n}\right)$ (observe (5.11)) satisfying:

$$
b_{c_{n}}^{+}(z)>t_{n}-d\left(z, c_{n}\left(t_{n}\right)\right) \text { and } s<t_{n}-d\left(z, c_{n}\left(t_{n}\right)\right) \text { for all } n \text { big enough. }
$$


That is, $d\left(z, c_{n}\left(t_{n}\right)\right)<t_{n}-s$, and there exists a curve $r:\left[s, t_{n}\right] \rightarrow M$ with $F(\dot{r})<1$ joining $z$ and $c_{n}\left(t_{n}\right)$. By Lemma $4.14 . \tau-d_{c(\tau)}^{+}$is increasing, and so,

$$
s-d_{z}^{+}<t-d_{r(t)}^{+}<t_{n}-d_{c_{n}\left(t_{n}\right)}^{+} \quad \forall t \in\left(s, t_{n}\right) .
$$

As $s-d_{z}^{+} \in C, t_{n}-d_{c_{n}\left(t_{n}\right)}^{+} \notin C$ and the functions $t-d_{r(t)}^{+}$vary continuously with $t$ in the pointwise convergence topology,

$$
\exists t_{n}^{z}: t_{n}^{z}-d_{c_{n}\left(t_{n}^{z}\right)}^{+} \in \partial C
$$

Summing up, we have found $t_{n}^{z}, y_{n}^{z}\left(=r\left(t_{n}^{z}\right)\right)$ such that: $t_{n}^{z}-d_{y_{n}^{z}}^{+} \in \partial C$ and $s-d_{z}^{+}<$ $t_{n}^{z}-d_{y_{n}^{z}}^{+}<t_{n}-d_{c_{n}\left(t_{n}\right)}^{+} \leq b_{c_{n}}^{+}$, the last inequality because of the Lipschitz condition (moreover, it is strict because of our choice $F(\dot{c})<1$, recall Remark 4.17). As $t_{n}^{z}-d_{y_{n}^{z}}^{+} \in \partial C \subset C$ compact, there exists $t^{z}, y^{z}$ such that $t^{z}-d_{y^{z}}^{+}$is the pointwise limit of a subsequence $\left\{t_{n_{k}}^{z}-d_{y_{n_{k}}^{z}}^{+}\right\}_{k}$. From the construction, $t^{z}, y^{z}$ satisfy the conditions (i) and (ii). And, as $t^{z}-d_{y^{z}}^{+}$is the pointwise limit of $t_{n_{k}}^{z}-d_{y_{n_{k}}^{z}}^{+} \leq b_{c_{n_{k}}}^{+}$, the condition (iii) is also satisfied, which ends the proof of the claim.

For the last assertion, observe that the essential properties used from $(M, d)$ have been: (1) the fact that $(M, d)$ is a length space; (2) the local compactness of $M$; (3) in order to prove (5.12), (5.13), the sentence below (5.11) and the convergence of $\left\{t_{n_{k}}^{z}-d_{y_{n_{k}}^{z}}^{+}\right\}_{k}$ above, we have used the continuity of the function $d$ in the second variable; (4) in order to prove 5.14, we have essentially used that any curve $c$ going from inside to outside the compact neighborhood $U$ of $x$ (which can be assumed to be a ball) must intersect the boundary of $U$, i.e. the continuity of $d$ in the first variable (recall that we consider backward balls in general). So, if we impose that $d_{Q}$ is a generalized distance and $M_{C}^{+}$is locally compact (thus, $M_{C}^{+}=M_{C}^{s}$ is a length space and the continuity in both variables is ensured; recall Proposition 3.28 and Lemma 5.5 applied to $d$ and $d^{\mathrm{rev}}$ ), the last assertion is obtained.

The required result is then:

Corollary 5.25 Let $(M, d)$ be under Convention 5.1 and $j_{B}^{+}: M_{C}^{+} \rightarrow M_{B}^{+}$as in (5.10). Then, $j_{B}^{+}$is injective and it is continuous if and only if the condition (a4') holds. The restriction $\left.j_{B}^{+}\right|_{M}$ is an open embedding and $j_{B}^{+}(M)$ is dense in $M_{B}^{+}$.

Moreover, if $d_{Q}$ is a generalized distance and $M_{C}^{+}$is locally compact, then $j_{B}^{+}$is also an embedding. In particular, this happens if $d$ is a (symmetric) distance and $M_{C}\left(=M_{C}^{+}\right)$is locally compact.

Proof. The continuity and injectivity of $j_{B}^{+}$follows as in Proposition 5.7 the density of $j_{B}^{+}(M)$ from Lemma 5.21 (ii), and $j_{B}^{+}(M)$ is open because $\Pi^{-1}\left(\partial_{B}^{+} M\right) \subset B^{+}(M)$ is closed for the chr. topology (for any sequence $\sigma$ in $\Pi^{-1}\left(\partial_{B}^{+} M\right), \hat{L}(\sigma)$ is also included in $\Pi^{-1}\left(\partial_{B}^{+} M\right)$ by Proposition 5.24). The continuity of the inverse of of $j_{B}^{+}$follows as a consequence of previous Proposition 5.24 and the fact that $M_{B}^{+}$is sequential (recall Remark 5.19). 
Remark 5.26 Notice that analogous results are also being obtained for $B^{+}(M)$. In particular, the map $\mathbb{R} \times M \hookrightarrow B^{+}(M),(t, x) \mapsto t-d_{x}^{+}$is an open embedding with dense image.

\subsubsection{Compactness of the Busemann completion}

Our aim is to prove that $M_{B}^{+}$provides a sequential compactification of $M$. To this objective, again we need a previous technical result.

Lemma 5.27 Assume that, for some $\phi \in \mathcal{L}_{1}(M, d), x_{0} \in M$ and $t_{0} \in \mathbb{R}$, the following subset of $B^{+}(M)$ is non-empty:

$$
\mathcal{F}=\left\{g \in B^{+}(M): g \leq \phi, g\left(x_{0}\right) \geq t_{0}\right\} .
$$

Then, $\mathcal{F}$ admits some maximal element (for the partial order $\leq$ ) in $B^{+}(M)$.

Proof. Applying Zorn's Lemma to $\mathcal{F}$, it suffices to show that any totally ordered subset admits an upper bound in $\mathcal{F}$. By contradiction, suppose that there exists a totally ordered subset $\mathcal{F}^{\prime}$ which has no upper bound. Associated to this set, there exists a countable family of functions $\left\{d_{\left(t_{n}, x_{n}\right)}^{+}\right\}$such that any $b_{c}^{+} \in \mathcal{F}^{\prime}$ is chronological limit of some increasing subsequence of $\left\{d_{\left(t_{n}, x_{n}\right)}^{+}\right\}$and each element $d_{\left(t_{n}, x_{n}\right)}^{+}$of the family admits some $b_{c}^{+} \in \mathcal{F}^{\prime}$ satisfying $d_{\left(t_{n}, x_{n}\right)}^{+}<b_{c}^{+}$. In fact, from Lemma 5.21 (ii), $b_{c}^{+}=\lim _{n} d_{\left(t_{n}^{c}, x_{n}^{c}\right)}^{+}$(pointwise, and then, chronologically), with $d_{\left(t_{n}^{c}, x_{n}^{c}\right)}^{+}<d_{\left(t_{n+1}^{c}, x_{n+1}^{c}\right)}^{+},\left(t_{n}^{c}, x_{n}^{c}\right) \in \mathbb{Q} \times D$ for all $n$, and $D$ a numerable, dense subset of $M$.

Construct a subsequence $\left\{d_{\left(s_{n}, y_{n}\right)}^{+}\right\}$of $\left\{d_{\left(t_{n}, x_{n}\right)}^{+}\right\}$by the following inductive way. Take $d_{\left(s_{1}, y_{1}\right)}^{+}:=d_{\left(t_{1}, x_{1}\right)}^{+}$, and given $d_{\left(s_{n}, y_{n}\right)}^{+}$, take $d_{\left(s_{n+1}, y_{n+1}\right)}^{+}$such that $d_{\left(s_{n+1}, y_{n+1}\right)}^{+}>$ $d_{\left(s_{n}, y_{n}\right)}^{+}$and $d_{\left(s_{n+1}, y_{n+1}\right)}^{+}>d_{\left(t_{i}, x_{i}\right)}^{+}, i=1, \ldots, n-1$. For the existence of $d_{\left(s_{n+1}, y_{n+1}\right)}^{+}$, note that, as $d_{\left(t_{i}, x_{i}\right)}^{+}<b_{c_{i}}^{+} \in \mathcal{F}^{\prime}, i=1, \ldots, n-1, d_{\left(s_{n}, y_{n}\right)}^{+}<b_{c_{n}}^{+} \in \mathcal{F}^{\prime}$ and $\mathcal{F}^{\prime}$ is totally ordered without upper bound, there exists $b_{c_{n+1}}^{+} \in \mathcal{F}^{\prime}$ such that $d_{\left(t_{i}, x_{i}\right)}^{+}<b_{c_{n+1}}^{+}, i=$ $1, \ldots, n-1$ and $d_{\left(s_{n}, y_{n}\right)}^{+}<b_{c_{n+1}}^{+}$; hence, the density of $\left\{d_{\left(t_{n}, x_{n}\right)}^{+}\right\}$in $\mathcal{F}^{\prime}$ ensures that such a $d_{\left(s_{n+1}, y_{n+1}\right)}^{+}$exists.

Finally, from Lemma 5.21 (i) one has that $d_{\left(s_{n+1}, y_{n+1}\right)}^{+}>d_{\left(s_{n}, y_{n}\right)}^{+}$implies $s_{n+1}-$ $s_{n}>d^{+}\left(y_{n}, y_{n+1}\right)$, and so, one can define a curve $\tau:\left[s_{1}, \Omega\right) \rightarrow M$, with $\Omega=\lim _{n} s_{n}$, such that $F(\dot{\tau})<1$ and $\tau\left(s_{n}\right)=y_{n}$. Moreover, the function $b_{\tau}^{+}$satisfies: $b_{\tau}^{+} \leq \phi$ (as $\left.d_{\left(s_{n+1}, y_{n+1}\right)}^{+} \leq \phi\right), b_{c}^{+} \leq b_{\tau}^{+}$for all $b_{c}^{+} \in \mathcal{F}^{\prime}$ (as $b_{\tau}^{+} \geq d_{\left(t_{n}, x_{n}\right)}^{+}$for all $n$ ) and $b_{\tau}^{+}\left(x_{0}\right) \geq b_{c}^{+}\left(x_{0}\right) \geq t_{0}$ (where $b_{c}^{+} \in \mathcal{F}^{\prime}$ ). Hence, $b_{\tau}^{+} \in \mathcal{F}$ is an upper bound of $\mathcal{F}^{\prime}$, in contradiction with our initial hypothesis.

Next, the required result on compactification is the following:

Theorem 5.28 The (forward) Busemann completion $M_{B}^{+}$of $(M, d)$ endowed with the chronological topology is a sequentially compact topological space. 
Proof. Consider any sequence $\sigma=\left\{\left[b_{c_{n}}^{+}\right]\right\}$in $M_{B}^{+}$. Pick a point $x_{0} \in M$ and, with no loss of generality, assume that $b_{c_{n}}^{+}\left(x_{0}\right)=0$. By Lemma 4.4 , there exists a subsequence $\left\{b_{c_{n_{k}}}^{+}\right\}_{k}$ with some pointwise limit $\phi \in \mathcal{L}_{1}(M, d)$ (in particular, $\phi\left(x_{0}\right)=$ 0 ). Putting $t_{0}=0$ in Lemma 5.21 (iii), $-d_{x_{0}}^{+} \leq \phi$ and the set $\mathcal{F}$ defined in 5.15 is non-empty. From Lemma 5.27, $\mathcal{F}$ must contain some maximal function $b_{c}^{+}$. This function is a chr. limit of $\left\{b_{c_{n_{k}}}^{+}\right\}_{k}$ according to 5.7). In fact, let $h \in B^{+}(M)$ with $b_{c}^{+} \leq h \leq \phi$. Necessarily, $h \in \mathcal{F}$ and by the maximality of $b_{c}^{+}, h=b_{c}^{+}$, i.e. $b_{c}^{+} \in \hat{L}\left(\left\{b_{c_{n_{k}}}^{+}\right\}_{k}\right)$. Therefore, $\left[b_{c}^{+}\right]$is also a limit of $\left\{\left[b_{c_{n_{k}}}^{+}\right]\right\}_{k}$, as required.

\subsubsection{The backward Busemann completion}

As always in the non-symmetric case, we can introduce "backward" definitions and results, just by using the reversed elements $F^{\text {rev }}, d^{\text {rev }}$ instead of $F, d$. However, there are some conventions (especially about signs) which suggest a different choice for the backward elements. These conventions will be very useful for the relations between the forward and backward Busemann boundaries provided by the c-boundary (see Section 6).

So, for the construction of the space $M_{B}^{-}$, we start with the map $j^{-}: x \mapsto$ $+d(x, \cdot)$ instead of the map $j^{+}: x \mapsto-d(\cdot, x)$. The space $C^{-}(M)$ is the set of all the piecewise smooth curves $c:[\alpha, \bar{\Omega}) \rightarrow M, \bar{\Omega} \leq \infty$, such that $F^{\mathrm{rev}}(\dot{c}) \leq 1$. Here, the endpoint of the interval has been written $\bar{\Omega}$, and we will put typically $\bar{\Omega}=-\Omega$. Now, the backward Busemann function is defined as $b_{c}^{-}(\cdot)=\lim _{s \rightarrow \bar{\Omega}}(-s+d(c(s), \cdot)) \in$ $\mathbb{R} \cup\{-\infty\}$, and $B^{-}(M)$ denotes the space of these functions which are finite. The backward chronological topology on $B^{-}(M)$ is introduced by means of the following limit operator $\breve{L}$, which replaces $\hat{L}$ in $(5.7)$ :

$$
f \in \check{L}(\sigma) \Longleftrightarrow\left\{\begin{array}{l}
(a) f \geq \limsup f_{n} \text { and } \\
(b) \forall g \in B^{-}(M) \text { with } f \geq g \geq \liminf _{n} f_{n}, \text { it is } g=f .
\end{array}\right.
$$

Recall that the corresponding notions of Gromov and Busemann completions, according to this alternative conventions, are totally equivalent, and so are the corresponding results.

\subsection{Chronological topology vs Gromov topology}

The Busemann completion and its different parts are regarded as topological spaces with the chr. topology. However, we have emphasized that $M_{B}^{+} \subset M_{G}^{+}$as a point set and, in fact, $j_{B}^{+}: M_{C}^{+} \rightarrow M_{B}^{+}$was just $j^{+}: M_{C}^{+} \rightarrow M_{G}^{+}$with the co-domain restricted at the point set level. We have already analyzed the relation between the Cauchy topology on $M_{C}^{+}$(induced from the backwards balls of the quasi-distance $\left.d_{Q}\right)$ and both, the Gromov and Busemann topologies on $j^{+}\left(M_{C}^{+}\right)\left(=j_{B}^{+}\left(M_{C}^{+}\right)\right)$, see Corollaries 5.16 and 5.25. Next, we are going to study the relations between the Gromov and Busemann topologies when the former is restricted to $M_{B}^{+}$. 


\subsubsection{The inclusion of $M_{B}^{+}$in $M_{G}^{+}$from the topological viewpoint}

The following result shows that the chr. topology is coarser than the Gromov one. As the latter is coarser than the Cauchy topology on $j^{+}\left(M_{C}^{+}\right)$, the chr. topology is the coarsest one of the three topologies.

Proposition 5.29 Consider a sequence $\left\{f_{n}\right\} \subset B^{+}(M)$ which converges pointwise to a function $f \in B^{+}(M)$. Then, $f$ is the unique chronological limit of $\left\{f_{n}\right\}$.

In particular, the inclusion $i: M_{B}^{+} \rightarrow M_{G}^{+}$satisfies that $i^{-1}: i\left(M_{B}^{+}\right) \rightarrow M_{B}^{+}$is continuous.

Proof. For the first assertion observe that, from the pointwise convergence of $\left\{f_{n}\right\}$, we have $\lim \sup f_{n}=\liminf f_{n}=f$. In particular, $\sigma=\left\{f_{n}\right\}$ and $f$ lie under the hypotheses of Proposition 5.20. Thus, $f$ is the unique limit of $\sigma$ in the chr. topology.

For the last one, as $M_{G}^{+}$is metrizable (Theorem 5.9 the continuity of $i^{-1}$ is characterized by sequences, so let $\left\{\left[f_{n}\right]\right\} \subset M_{B}^{+}$which converges pointwise to $[f] \in$ $M_{B}^{+}$. With no loss of generality, we can assume that $\left\{f_{n}\right\}$ converges pointwise to $f$. Then, $f$ is the (unique) chr. limit of $\left\{f_{n}\right\}$ from the first part, and the required chr. convergence of $\left\{\left[f_{n}\right]\right\}$ to $[f]$ follows from the continuity of the natural projection $\Pi: B^{+}(M) \rightarrow M_{B}^{+}$.

Remark 5.30 The differences between the Busemann and Gromov topologies at the point set level appeared clearly even in the Riemannian case, see Remark 4.18. It is easy to check that, in these cases, there is also a difference at the topological level. In fact, in Figure 4 (A) the sequence $\left\{\left[-d_{x_{n}}^{R}\right]\right\}_{n}$ converges to both, $\left[-d_{(0,0)}^{R}\right]$ and $\left[-d_{(0,1)}^{R}\right]$ (recall Fig. $4(\mathrm{~B})$ ). As we will see, there are differences at the point set level occur if and only if the Busemann topology is different (strictly coarser) than the induced by Gromov one in $M_{B}^{+}$(see Remark 5.41).

Therefore, in general the chr. topology is strictly coarser than Gromov one. Recall also that Example 4.9 also showed that the Gromov topology (and then, the Busemann one) is strictly coarser than the Cauchy topology.

\subsubsection{The case of separating topologies}

Most of the difficulties of the chr. topology come from the fact that the limit operator $\hat{L}$ may be not of the first order. We will see next that a simple hypotheses prevents this case and ensures automatically quite a few of nice properties. These hypotheses are adapted from a natural one for the c-boundary of spacetimes, which was studied in [12, Defn. 3.42, Prop. 3.44]. Recall that the open subsets $I^{+}\left(p_{0}, B^{+}(M)\right)$ were defined in 5.9 .

Definition 5.31 The (forward) chr. topology on $B^{+}(M)$ is separating if the following property holds: for any two functions $f, f^{\prime} \in B^{+}(M)$, with $f^{\prime} \leq f, f^{\prime} \neq f$, there exists some $p_{0}=\left(t_{0}, x_{0}\right) \in \mathbb{R} \times M$ which separates them, i.e., $p_{0}$ satisfies $f^{\prime} \notin \overline{I^{+}\left(p_{0}, B^{+}(M)\right)}$ but $f^{\prime}\left(x_{0}\right)<t_{0}<f\left(x_{0}\right)$ (and thus, $f \in I^{+}\left(p_{0}, B^{+}(M)\right)$ ). 
Proposition 5.32 If $B^{+}(M)$ (endowed with the chr. topology) is separating, then $B^{+}(M)$ is second countable and $\hat{L}$ is of first order. Therefore, $M_{B}^{+}$is also second countable.

Proof. First, let us prove that the following family of subsets in $B^{+}(M)$ is a (countable) topological basis:

$$
\Omega=\left\{I^{+}\left(p_{n}, B^{+}(M)\right) \cap{\overline{\cup_{i=1}^{k} I^{+}\left(p_{j_{i}}, B^{+}(M)\right)}}^{c}: n, j_{i} \in \mathbb{N}\right\},
$$

where $D=\left\{p_{n}\right\}_{n}$ is a dense countable subset of $\mathbb{R} \times M$. Consider $f^{\prime} \in B^{+}(M)$, choose a chain $\left\{d_{p_{n}^{\prime}}^{+}\right\}$converging pointwise to $f^{\prime}$ with $\left\{p_{n}^{\prime}\right\} \subset D$ (recall Lemma 5.21(ii)) and construct a basis $\left\{U_{n}\right\} \subset \Omega$ of open neighborhoods of $f^{\prime}$ as follows. Let $\left\{s_{i}=p_{n_{i}}\right\}_{i} \subset D$ be the (countable) subset composed by those points in $D$ which separate $f^{\prime}$ and $f$, for some $f \in B^{+}(M)$ such that $f^{\prime} \leq f, f^{\prime} \neq f$. Recall about this subset that, from the separation property assumed in the hypotheses, for any $f$ as above there exists some $p_{0}=\left(t_{p_{0}}, x_{p_{0}}\right) \in \mathbb{R} \times M$ which separates $f^{\prime}$ and $f$. Thus, any $s=\left(t_{s}, x_{s}\right) \in D$ satisfying $d\left(x_{p_{0}}, x_{s}\right)<t_{s}-t_{p_{0}}$ and $t_{s}<f\left(x_{s}\right)$ provides an element of $\left\{s_{i}\right\}$ associated to $f$ (recall that necessarily $d_{p_{0}}^{+}<d_{s}^{+}$, then $I^{+}\left(s, B^{+}(M)\right) \subset I^{+}\left(p_{0}, B^{+}(M)\right)$ and this inclusion holds for the closures, i.e., $f^{\prime} \notin$ $\left.\overline{I^{+}\left(s, B^{+}(M)\right)}\right)$. The density of $D$ allows to find (infinitely many) such a $s$ with $d\left(x_{p_{0}}, x_{s}\right)$ small enough, and the full $\left\{s_{i}\right\}$ is constructed by taking all such $s$ for all such functions $f$. Now, define each $U_{n} \in \Omega$ as $U_{n}:=I^{+}\left(p_{n}^{\prime}, B^{+}(M)\right) \cap A_{n}$, where $A_{n}={\overline{\cup_{i=1}^{n} I^{+}\left(s_{i}, B^{+}(M)\right)}}^{c}$. Since $f^{\prime} \notin \overline{I^{+}\left(s_{i}, B^{+}(M)\right)}$ for all $i$, the open subsets $U_{n}$ contain $f^{\prime}$.

To check that $\left\{U_{n}\right\}$ constitute the required basis around $f^{\prime}$, let us prove first that any sequence $\left\{g_{n}\right\}$ such that $g_{n} \in U_{m}$ for all $m$ and all $n>n(m)$, satisfies $f^{\prime} \in \hat{L}\left(\left\{g_{n}\right\}\right)$. In fact, to check the first condition in (5.7), recall that $g_{n} \in I^{+}\left(p_{m}^{\prime}, B^{+}(M)\right)$, and so, $g_{n}>d_{p_{m}^{\prime}}^{+}$. As $d_{p_{m}^{\prime}}^{+} \rightarrow f^{\prime}$ pointwise, the inequality $f^{\prime} \leq \liminf _{n} g_{n}$ holds. For the second condition in (5.7), if $f^{\prime} \leq f \leq \lim \sup _{n} g_{n}$, $f^{\prime} \neq f$, then $f \in I^{+}\left(s_{i_{0}}, B^{+}(M)\right)$ for some $s_{i_{0}} \in\left\{s_{i}\right\}$ and, thus, $t_{s_{i_{0}}}<f\left(x_{s_{i_{0}}}\right)$. As $f \leq \limsup _{n} g_{n}$, then $t_{s_{i_{0}}}<g_{n}\left(x_{s_{i_{0}}}\right)$, i.e., $g_{n} \in I^{+}\left(s_{i_{0}}, B^{+}(M)\right)$, for infinitely many $n$, in contradiction with the hypothesis $g_{n} \in U_{m}$. Now, it is clear that if some neighborhood $U$ of $f^{\prime}$ satisfied $U_{n} \not \subset U$ for all $n$, the sequence $\left\{g_{n}\right\}$ obtained by choosing some $g_{n} \in U_{n} \backslash U$ for all $n$ would not converge to $f^{\prime}$ with the chr. topology but $f^{\prime} \in \hat{L}\left(\left\{g_{n}\right\}\right)$, an absurd (see Remark 5.19).

The first order property of $\hat{L}$ is also straightforward now, because if $\sigma=\left\{g_{n}\right\}$ converges to $f^{\prime}$ with the chr. topology, necessarily $g_{n} \in U_{m}$ for all $m$ and all $n \geq n(m)$, and then $f^{\prime} \in \hat{L}\left(\left\{g_{n}\right\}\right)$.

For the last assertion, just use Proposition 5.23 and project the basis 5.16 .

Remark 5.33 As we will see, if $B^{+}(M)$ is Hausdorff then it is separating (just apply Remark 5.35 and Proposition 5.37), but the converse does not hold. In the framework of spacetimes, it is easy to find examples of causal completions such that its (chr.) topology is not separating and, even more, non-first countable, see [12, Example 3.43, Remark 3.40]. So, it is conceivable that these properties may occur 
for $B^{+}(M)$ (and, then, be transmitted to $M_{B}^{+}$), even though we do not have any example where 5.16 is not a basis for the chr. topology.

\subsubsection{Topologies with unique limits of sequences}

As we have seen, $M_{B}^{+}$may be non-Hausdorff and this is a clear difference with $M_{G}^{+}$. Our aim will be to show that only in this case there are differences between both topologies. However, we will study the non-Hausdorff character from a more general viewpoint (compare with [33]).

Definition 5.34 Let $X$ be a set. A topology on $X$ is unique limit for sequences $(U L S)$ if any sequence in $X$ has at most one limit. A topology $\tau_{L}$ associated to a limit operator $L$ on $X$ is unique limit for $L$ (ULL) if, for any sequence $\sigma$ in $X$, $L(\sigma)$ contains at most one element.

Remark 5.35 Clearly, Hausdorff $\Rightarrow$ ULS $\Rightarrow$ ULL (recall Remark 5.19p and, for first countable spaces, ULS $\Rightarrow$ Hausdorff.

Now, let us consider again $B^{+}(M)$ with the chr. topology - always regarded as the topology associated to $\hat{L}$.

Lemma 5.36 Assume that $B^{+}(M)$ is ULL. If a sequence $\left\{b_{c_{n}}^{+}\right\} \subset B^{+}(M)$ converges pointwise to some Lipschitz function $\phi \in \mathcal{L}_{1}(M, d)$ and $b_{c}^{+} \in \hat{L}\left(\left\{b_{c_{n}}^{+}\right\}\right)$, then $\phi=b_{c}^{+}$.

Proof. Since $b_{c}^{+} \in \hat{L}\left(\left\{b_{c_{n}}^{+}\right\}\right)$, necessarily $b_{c}^{+} \leq \phi$. Assuming by contradiction that $\phi \neq b_{c}^{+}$, there is some $x_{0} \in M$ and $t_{0} \in \mathbb{R}$ with $b_{c}^{+}\left(x_{0}\right)<t_{0}<\phi\left(x_{0}\right)$. Thus, for $p_{0}=\left(t_{0}, x_{0}\right)$, necessarily $d_{p_{0}}^{+}<\phi$ (recall Lemma 5.21 (iii)), and so $d_{p_{0}}^{+} \in \mathcal{F}$, i.e. the set $\mathcal{F}$ constructed in $(5.15)$ is non-empty. Then, Lemma 5.27 ensures that $\mathcal{F}$ must contain a maximal function $b_{\tau}^{+}$and, even more, $b_{\tau}^{+} \in \hat{L}\left(\left\{b_{c_{n}}^{+}\right\}\right.$) (for any $h \in B^{+}(M)$ with $b_{\tau}^{+} \leq h \leq \phi$, we have $h \in \mathcal{F}$; since $b_{\tau}^{+}$is maximal, necessarily $h=b_{\tau}^{+}$, and thus, $\left.b_{\tau}^{+} \in \hat{L}\left(\left\{b_{c_{n}}^{+}\right\}\right)\right)$. But since $b_{\tau}^{+}\left(x_{0}\right) \geq t_{0}>b_{c}^{+}\left(x_{0}\right)$, we know $b_{\tau}^{+} \neq b_{c}^{+}$, in contradiction to the ULL character of $B^{+}(M)$.

Proposition 5.37 If $B^{+}(M)$ is ULL, then it is separating. So, $\hat{L}$ is of first order and both, the chr. topologies on $B^{+}(M)$ and $M_{B}^{+}$, are $U L S$, second countable and Hausdorff.

Proof. To prove that $B^{+}(M)$ is separating, let $f^{\prime}, f \in B^{+}(M)$, with $f^{\prime} \leq f$ and $f^{\prime}\left(x_{0}\right)<t_{0}<f\left(x_{0}\right)$ for some $p_{0}=\left(t_{0}, x_{0}\right)$, and let us prove that $f^{\prime} \notin$ $\overline{I^{+}\left(p_{0}, B^{+}(M)\right)}$ (recall $\left.(5.9)\right)$. Note that it suffices to check that the subset $\{g \in$ $\left.B^{+}(M): t_{0} \leq g\left(x_{0}\right)\right\}$ (which does not contain $f^{\prime}$ ), is chr. closed. Reasoning by contradiction, assume that there exists some sequence $\sigma=\left\{f_{n}\right\}$, with $t_{0} \leq f_{n}\left(x_{0}\right)$ for all $n$, and $h \in \hat{L}(\sigma)$, such that $h\left(x_{0}\right)<t_{0}$. As $M_{G}^{+}$is compact, there exist constants $t_{n} \in \mathbb{R}$ and a subsequence $\left\{f_{n_{k}}+t_{k}\right\}$ converging pointwise to some Lipschitz function $\phi^{\prime}$. It is not a restriction to suppose that this subsequence satisfies $t_{k} \rightarrow t_{\infty}$ for some $t_{\infty} \in \mathbb{R}$. In fact, otherwise, for some subsequence $\left\{t_{k_{l}}\right\}_{l}$ we 
have either $t_{k_{l}} \rightarrow \infty$, and so, as $t_{0} \leq f_{n}\left(x_{0}\right), \phi^{\prime} \equiv \infty$ (an absurd), or $t_{k_{l}} \rightarrow-\infty$ and, then, $f_{n_{k_{l}}}\left(x_{0}\right) \rightarrow \infty$, which, by Remark 5.3(2), implies $\liminf \left\{f_{n_{k_{l}}}\right\}=\infty$, in contradiction with the maximality of $h$ in $\lim \sup \left\{f_{n}\right\}$ required for the elements of $\hat{L}(\sigma)$. Therefore, $\bar{\sigma}=\left\{f_{n_{k}}\right\}$ converges pointwise to $\phi=\phi^{\prime}-t_{\infty}$. In particular, $h\left(x_{0}\right)<t_{0} \leq \phi\left(x_{0}\right)$. Since $h \in \hat{L}(\bar{\sigma})$, this contradicts Lemma 5.36 .

For the last assertions recall that, as $B^{+}(M)$ is separating, Proposition 5.32 ensures that the chr. topology is second countable and $\hat{L}$ is of first order. Moreover, as $B^{+}(M)$ is ULL, the chr. topology is ULS and, so, Hausdorff (recall Remark 5.35).

\subsubsection{Main results}

The following theorems show the precise relation between the Busemann and Gromov completions. The following technical lemma is required first.

Lemma $5.38 B^{+}(M)$ is ULL if and only if $M_{B}^{+}$is Hausdorff.

Proof. The implication to the right is included in Proposition 5.37. For the implication to the left, assume that $B^{+}(M)$ is not ULL. Then, $\hat{L}(\sigma)$ contains two distinct elements for some sequence $\sigma \subset B^{+}(M)$. From property (b) in (5.7), the difference between these elements is not a constant. So, they define two different classes of $M_{B}^{+}$, and thus, $M_{B}^{+}$is not Hausdorff.

Theorem 5.39 The following statements are equivalent:

(a) No sequence in $M_{B}^{+}$converges to more than one point in $\partial_{B}^{+} M$ with the forward chronological topology. For any sequence $\sigma \subset M_{B}^{+}, L(\sigma)$ contains at most one point in $\partial_{B}^{+} M$.

(b) $M_{B}^{+}$is Hausdorff with the forward chronological topology.

(c) $i: M_{B}^{+} \rightarrow M_{G}^{+}$is continuous (and thus, an embedding).

(d) $M_{B}^{+}=M_{G}^{+}$(both, as a point set and topologically).

(e) $M_{B}^{+}$is equal to $M_{G}^{+}$as a point set.

Proof. $(a) \Rightarrow(b)$. Suppose by contradiction that $M_{B}^{+}$is not Hausdorff, and thus, $B^{+}(M)$ is not ULL (recall Lemma 5.38). So, there exists a sequence $\left\{b_{c_{n}}^{+}\right\}_{n}$ such that $\hat{L}\left(\left\{b_{c_{n}}^{+}\right\}\right)$contains two distinct elements of $B^{+}(M)$. By hypothesis (a), one of these functions is of the form $\Omega-d_{x}^{+}$with $x \in M$. From Proposition $5.24\left\{b_{c_{n}}^{+}\right\}_{n}$ converges pointwise to $\Omega-d_{x}^{+}$. But, then, Proposition 5.29 ensures that this is the unique limit, a contradiction.

$(b) \Rightarrow(c)$. Since $M_{B}^{+}$is a sequential space the continuity of $i$ is characterized by means of sequences (recall Remark 5.19); by Proposition 5.29, $i$ will be then also an embedding. So, assume that $\left\{\left[b_{c_{n}}^{+}\right]\right\}$converges with the forward chronological topology to $\left[b_{c}^{+}\right]$. It is not a restriction to assume that $\left\{b_{c_{n}}^{+}\right\}$converges with the forward chronological topology to $b_{c}^{+}$. As $M_{B}^{+}$is Hausdorff, $B^{+}(M)$ is ULL (Lemma 
5.38), and thus, $\hat{L}$ is of first order (Proposition 5.37). So, we can also assume that $b_{c}^{+} \in \hat{L}\left(\left\{b_{c_{n}}^{+}\right\}\right)$and, reasoning by contradiction, that $\left\{b_{c_{n}}^{+}\right\}$does not converge pointwise to $b_{c}^{+}$. Moreover, there exists a subsequence $\left\{b_{c_{n_{k}}}^{+}\right\}_{k}$ converging pointwise to some $\phi \neq b_{c}^{+}$(reason as in Proposition 5.37, namely, for any $x_{0} \in M$, the sequence $\left\{\left|b_{c_{n_{k}}}^{+}\left(x_{0}\right)\right|\right\}_{k}$ must be bounded because, otherwise, a contradiction with $\hat{L}\left(\left\{b_{c_{n}}^{+}\right\}\right) \neq \emptyset$ would appear). This is in contradiction with Lemma 5.36, since $b_{c}^{+}$ belongs also to $\hat{L}\left(\left\{b_{c_{n_{k}}}^{+}\right\}_{k}\right)$.

$(c) \Rightarrow(d)$. Any point $[f] \in M_{G}^{+}$is the pointwise limit of some sequence $\left\{j^{+}\left(x_{n}\right)\right\} \subset M_{B}^{+}$, with $\left\{x_{n}\right\} \subset M$. As $M_{B}^{+}$is sequentially compact, up to a subsequence, $\left\{j^{+}\left(x_{n}\right)\right\}$ converges with the forward chronological topology to some $[\bar{f}] \in M_{B}^{+}$. By the continuity of $i,\left\{j^{+}\left(x_{n}\right)\right\}$ also converges pointwise to $[\bar{f}]$, and so, $[\bar{f}]=[f]$. So, $i$ is onto and, under our hypotheses, a homeomorphism.

$(d) \Rightarrow(e)$. Trivial.

$(e) \Rightarrow(a)$. By contradiction, assume that $\left\{\left[b_{c_{n}}^{+}\right]\right\} \subset M_{B}^{+}$converges with the chr. topology to distinct elements $\left[b_{c}^{+}\right] \neq\left[b_{c^{\prime}}^{+}\right]$of $\partial_{B}^{+} M$. By the compactness of $M_{G}^{+}$and hypothesis (e), there exists some subsequence $\left\{b_{c_{n_{k}}^{+}}^{+}\right\}_{k}$ converging pointwise to some $[f] \in M_{G}^{+}=M_{B}^{+}$. From Proposition 5.29, $[f]$ must be the unique chronological limit of $\left\{\left[b_{c_{n_{k}}}^{+}\right]\right\}_{k}$, and thus of $\left\{\left[b_{c_{n}}^{+}\right]\right\}$, in contradiction with the initial assumption.

Recall that Gromov and Eberlein and O'Neill's boundaries coincide, both as a point set and topologically, when $(M, g)$ is a Hadamard manifold [2, 5. So, Theorem 5.39 also ensures the equality with the Busemann boundary.

Corollary 5.40 Let $(M, g)$ be a Hadamard manifold. Then the Busemann and Gromov compactifications (as well as Eberlein and O'Neill's one) coincide.

Proof. As Eberlein and O'Neill's compactification is carried out by means of Busemann functions of rays, then it is always included in $M_{B}$ as a point set. So, as that compactification agrees with Gromov's one in a Hadamard manifold, $M_{G}=M_{B}$ as point sets and, from Theorem 5.39, also topologically.

Remark 5.41 As emphasized in Remark 4.18 and Figure 5 , it is not difficult to find examples with $M_{B} \neq M_{G}$ as a point set -and, as a consequence of Theorem 5.39, where $i: M_{B}^{+} \rightarrow M_{G}^{+}$is neither an embedding. This happens when there exists some point in $M_{G}$ that cannot be reached from $M$ by means of an asymptotically raylike curve, i.e. a curve with finite Busemann function. Perhaps the most dramatic difference happens when some points in $\partial_{G} M$ cannot be reached as limits of any type of curves in $M$ (recall Figure $4(\mathrm{~A})$ ). But, even when they can, it is not difficult to construct examples where the Gromov completion differs from Busemann one.

The example depicted in Figure 6 may be especially illustrating. Here, if one modifies the usual metric of a cylinder by putting the depicted "chimney" (Figure 6 (A)), then a point appears for both, the Gromov and Busemann boundaries (as well as for the Cauchy one). If two such chimneys are included (Figure 6 (B)), two points appear for $\partial_{B} M$ (and $\partial_{C} M$ ), but a continuum of points (a segment) appears for $\partial_{G} M$. The two points in $\partial_{B} M$ are not Hausdorff separated for the chr. topology. This reflects the fact that $\partial_{B} M$ is a compactification, not only a 
completion (as $\partial_{C} M$ was). The "surprising" appearance of a continuum of points for $\partial_{G} M$ is a consequence of the Hausdorffness of the Gromov compactification.

It is also worth pointing out that, in the Figure 6 (B), the induced topology in $\partial_{B} M$ from $M_{B}$ is the discrete one (this is a consequence of the fact that the chr. topology is $T_{1}$ and $\partial_{B} M$ contains only two points). So $\partial_{B} M$ is Hausdorff, even though the two points of $\partial_{B} M$ are not Hausdorff related as points of $M_{B}$. Analogously, local compactness is not satisfied (neither in the case (A) nor in (B) of Figure 6) by the points of $\partial_{C} M$ regarded as points of $M_{C}$, but it is satisfied in $\partial_{C} M$ regarded as a topological space.

\subsection{Proof of Theorem 1.1}

Now, we are in conditions to summarize the proof of Theorem 1.1 .

Proof of Theor. 1.1. Point 1. It follows from Theorem 5.9.

Point 2. The sequential compactness is proved in Theorem 5.28. For the $T_{1}$ character of the chr. topology, recall Remark 5.19. The non-Hausdorff possibility (as well as the possible non-continuity of the inclusion $i$ in the second paragraph), are illustrated by Figure 4 (Remark 5.30). The continuity of $i^{-1}$ in the second paragraph is proved in Proposition 5.29 The third paragraph is included in Theorem 5.39.

Point 3. (First paragraph). The character of quasi-distance is ensured by Proposition 3.25 and the $T_{0}$ property by Theorem 3.29 .

Point 3. (A) The first paragraph follows from Proposition 5.7. For the second paragraph, the local compactness of $M_{C G}^{+}$follows from the compactness of $M_{G}^{+}$ and the Heine-Borel property is proved in Corollary 5.16 (2). The third paragraph collects Corollary 5.16 (3).

Point 3. (B) follows from Corollary 5.25.

Point 3. (Last paragraph). Direct consequence of Theorem 3.29 Proposition 3.30. Theorem 5.9 and Corollary 5.25 (for the last assertion, use also Theorem 5.39).

\section{C-boundary of standard stationary spacetimes}

Next, we are going to use the structures introduced in previous sections to describe the c-boundary of standard stationary spacetimes. To this aim, we will consider spacetimes as in 2.4 with the choice of conformal factor $\Lambda=1$, i.e.

$$
V=\mathbb{R} \times M \quad \text { and } \quad g=-d t^{2}+\pi^{*} \omega \otimes d t+d t \otimes \pi^{*} \omega+\pi^{*} g_{0} .
$$

The natural projection $t: \mathbb{R} \times M \rightarrow \mathbb{R}$ will be called the (standard) time, -and we will use the letter $t$ with some obvious abuse of notation. From this metric, we consider the Fermat metrics $F^{ \pm}=\sqrt{g_{0}+\omega^{2}} \pm \omega$ as in 2.5 . We will denote with subscripts $+($ resp. -$)$ the objects associated to the metric $F^{+}$(resp. $F^{-}$). So, the associated generalized distance will be denoted $d^{+}$and its reverse distance $d^{-}$. $\left(M, d^{ \pm}\right)$will be the generalized metric spaces associated to the standard stationary spacetime. 


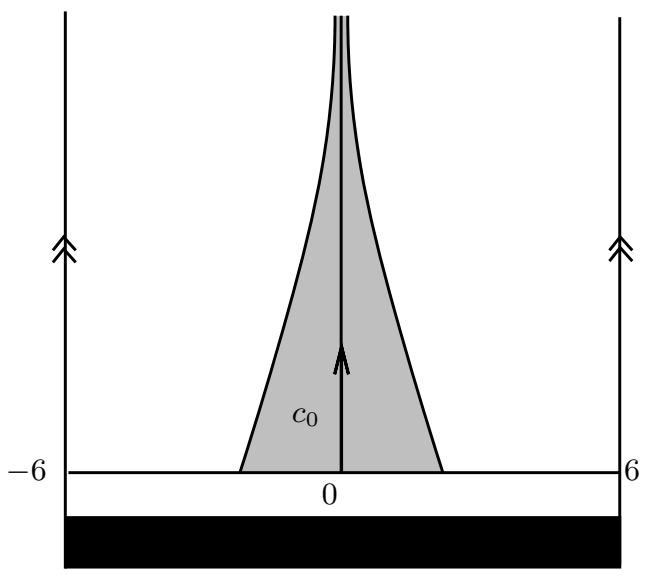

(A)

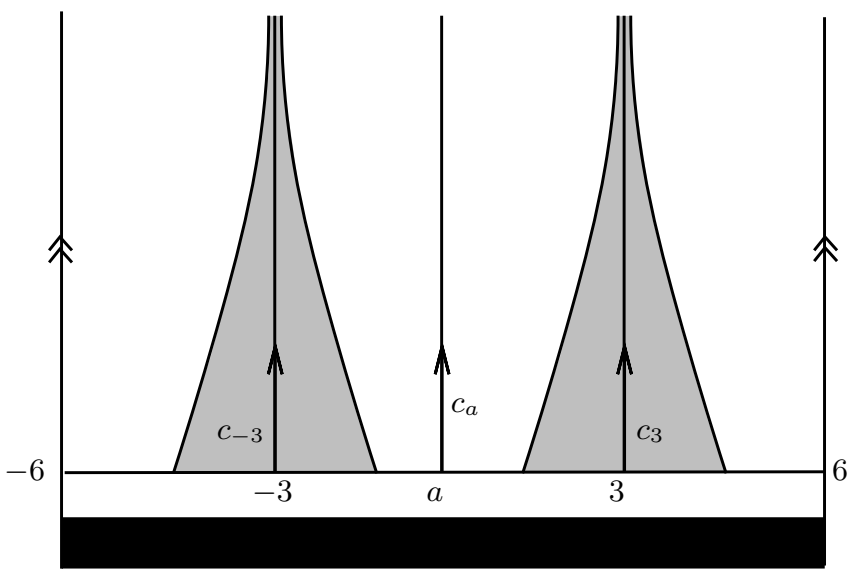

(B)

Figure 6: Non-Hausdorff $\partial_{B} M$ and surprising points in $\partial_{G} M$ (Remark 5.41).

Starting at a strip on the plane, consider the half cylinder $M=[-6,6] \times(-1, \infty) / \sim$ where $\sim$ identifies each $(-6, y)$ with $(6, y)$, for all $y>-1$ (the black regions are irrelevant for our problem, and will not be taken into account). Put a Riemannian metric (which can be chosen conformal to the usual Euclidean one) in such a way that: (i) out of the "chimneys" (the regions drawn in grey) the metric is the Euclidean one, and (ii) the central curves of the chimneys $\left(c_{0}\right.$ in the case $(\mathrm{A})$ and $c_{-3}, c_{3}$ in the case $\left.(\mathrm{B})\right)$ have finite length.

Case (A): $M_{B}$ and $M_{G}$ coincide (as, obviously, no sequence in $M$ can converge to two distinct points of $\left.\partial_{B} M\right)$. Moreover, the corresponding boundary is naturally identified with $\left\{x_{0}\right\}$, where $x_{0}$ is the Cauchy boundary point associated to the curve $c_{0}$.

Case (B): The Cauchy and Busemann boundaries coincide as point sets and are identifiable with $\left\{x_{-3}, x_{3}\right\}$, where $x_{-3}, x_{3}$ are the Cauchy boundary points associated to $c_{-3}$ and $c_{3}$ resp. Nevertheless, the Gromov boundary include also a point for any curve of the form $c_{a}(t)=(a, t)$ with $a \in[-3,3]$, i.e., the Gromov boundary naturally includes a "segment" which connects $x_{-3}$ and $x_{3}$. 


\subsection{Chronological relations and Lipschitzian functions}

In this subsection we are going to establish the chronological relations of $(V, g)$ in terms of the Finsler metrics $F^{ \pm}$. The arguments are inspired by the seminal work of Harris for the standard static case [20].

Proposition 6.1 Consider two points $\left(t_{0}, x_{0}\right),\left(t_{1}, x_{1}\right) \in V$. Then:

$$
\left(t_{0}, x_{0}\right) \ll\left(t_{1}, x_{1}\right) \Longleftrightarrow d^{+}\left(x_{0}, x_{1}\right)<t_{1}-t_{0} .
$$

Proof. If $\left(t_{0}, x_{0}\right) \ll\left(t_{1}, x_{1}\right)$ then there exists a future-directed timelike curve $\gamma(s)=$ $(t(s), c(s))$ such that $\gamma(0)=\left(t_{0}, x_{0}\right), \gamma(1)=\left(t_{1}, x_{1}\right)$. Since $\gamma$ is future-directed timelike, necessarily $\dot{t}(s)>\sqrt{g_{0}(\dot{c}(s), \dot{c}(s))+\omega(\dot{c}(s))^{2}}+\omega(\dot{c}(s))=F^{+}(\dot{c}(s))$ for all $s$. In particular,

$$
t_{1}-t_{0}=\int_{0}^{1} \dot{t}(s) d s>\int_{0}^{1} F^{+}(\dot{c}(s))=\operatorname{length}_{+}(c) \geq d^{+}\left(x_{0}, x_{1}\right) .
$$

Reciprocally, if $t_{1}-t_{0}>d^{+}\left(x_{0}, x_{1}\right)$ then there exists a curve $c(s)$ in $M$ with $c(0)=$ $x_{0}, c_{1}(1)=x_{1}$ and constant speed $F^{+}(\dot{c}(s))<t_{1}-t_{0}$. Therefore, the curve $\gamma(s)=$ $(t(s), c(s))$, with $t(s)=\left(t_{1}-t_{0}\right) s+t_{0}$, is timelike, future-directed and satisfies $\gamma(0)=\left(t_{0}, x_{0}\right), \gamma(1)=\left(t_{1}, x_{1}\right)$, as required.

Notice that we can reparametrize the timelike curves by using the standard time $t$. Therefore, from the expression of the standard stationary metric $g$ in 6.1 , we deduce directly:

Proposition 6.2 A curve $\gamma(t)=(t, c(t))$ (resp. $\gamma(t)=(-t, c(t))), t \in I$ (interval), is future (resp. past) directed timelike if and only if $F^{+}(\dot{c})<1$ (resp. $\left.F^{-}(\dot{c})<1\right)$.

As a consequence, the past and future sets of $V$ (according to the definitions in Section 2.1 can be characterized by means of Lipschitz functions. More precisely:

Definition 6.3 Let $f$ be a Lipschitz function for $d^{+}$(resp. $\left.d^{-}\right)$. Its past $P(f)$ (resp. future $F(f)$ ) is the following subset of $V=\mathbb{R} \times M$ :

$$
P(f):=\{(t, x) \in V: t<f(x)\} \subset V \quad(\text { resp. } F(f):=\{(t, x) \in V: t>f(x)\} \subset V) .
$$

Proposition 6.4 A (non-empty) subset $P, P \varsubsetneqq V$ (resp. $F, F \varsubsetneqq V$ ) is a past (resp. future) set if and only if $P=P(f)$ (resp. $F=F(f)$ ) for some Lipschitz function $f$ for $d^{+}$(resp. for $d^{-}$).

Proof. For the implication to the right, given $P$ define

$$
f(x):=\sup \{t \in \mathbb{R}:(t, x) \in P\}, \quad \forall x \in M,
$$

and choose two points $\left(t_{0}, x_{0}\right) \notin P,\left(t_{1}, x_{1}\right) \in P$. As $d^{+}\left(x, x_{1}\right)<t_{1}-t$ for large $-t$, necessarily $f(x)>-\infty$. Moreover, $f(x) \leq t_{0}+d^{+}\left(x_{0}, x\right)$ because, otherwise, any $t \in\left(t_{0}+d^{+}\left(x_{0}, x\right), f(x)\right)$ would yield the contradiction $\left(t_{0}, x_{0}\right) \ll(t, x) \in P$. In particular, $f(x)<\infty$ and 6.2 defines a function $f: M \rightarrow \mathbb{R}$. As $P$ is a past 
set, observe that any pair $(t, x)$ with $t<f(x)$ belongs to $P$ (if $t<f(x)$, there exists $t^{\prime}$ such that $t<t^{\prime}<f(x)$ and $\left.(t, x) \ll\left(t^{\prime}, x\right) \in P\right)$. Furthermore, $f$ is Lipschitz (so, we can write $P=P(f)$ according to Defn. 6.3). In fact, otherwise $f(y)>f(x)+d^{+}(x, y)$ for some $x, y$. Picking some $t \in\left(f(x)+d^{+}(x, y), f(y)\right)$ we have $(f(x), x) \ll(t, y) \in P$, in contradiction to the facts that $P$ is a past set and $(f(x), x) \notin P(P$ must be open $)$.

For the implication to the left, it suffices to prove that $P(f)=I^{-}[P(f)]$. For the inclusion $\subseteq$, take any $(t, x) \in P(f)$. Then, $t<f(x)$, and so, there exists $t^{\prime}$ such that $t<t^{\prime}<f(x)$. In particular, $(t, x) \ll\left(t^{\prime}, x\right) \in P(f)$, since $t^{\prime}-t>0=d^{+}(x, x)$. For the inclusion $\supseteq$, suppose that $(t, x) \in I^{-}[P(f)]$. Then, there exists some point $\left(t^{\prime}, x^{\prime}\right) \in P(f)$ such that $(t, x) \ll\left(t^{\prime}, x^{\prime}\right)$, or, equivalently, $d^{+}\left(x, x^{\prime}\right)<t^{\prime}-t$. Assume by contradiction that $(t, x) \notin P(f)$. Then, $t \geq f(x)$. This joined to the fact that $\left(t^{\prime}, x^{\prime}\right) \in P(f)$, i.e. $t^{\prime}<f\left(x^{\prime}\right)$, implies

$$
t^{\prime}-t<f\left(x^{\prime}\right)-f(x) \leq d^{+}\left(x, x^{\prime}\right),
$$

the latter inequality because $f$ is Lipschitz.

Convention 6.5 As $V$ is also a past and future set, we put $P(\infty)=F(-\infty):=V$.

\subsection{Future and past c-boundaries as point sets}

Next, we are going to construct the partial (future and past) c-boundaries. Consider a future-directed timelike curve $\gamma:[\alpha, \Omega) \rightarrow V$ parametrized as $\gamma(t)=(t, c(t))$. By using that function $t \mapsto t-d^{+}(\cdot, c(t))$ is increasing (recall (5.5)), the past of $\gamma$ can be expressed in the following way:

$$
\begin{aligned}
I^{-}[\gamma] & =\left\{\left(t^{\prime}, x^{\prime}\right) \in V:\left(t^{\prime}, x^{\prime}\right) \ll \gamma(t) \text { for some } t \in[\alpha, \Omega)\right\} \\
& =\left\{\left(t^{\prime}, x^{\prime}\right) \in V: t^{\prime}<t-d^{+}\left(x^{\prime}, c(t)\right) \text { for some } t \in[\alpha, \Omega)\right\} \\
& =\left\{\left(t^{\prime}, x^{\prime}\right) \in V: t^{\prime}<\lim _{t \rightarrow \Omega}\left(t-d^{+}\left(x^{\prime}, c(t)\right)\right)\right\} \\
& =\left\{\left(t^{\prime}, x^{\prime}\right) \in V: t^{\prime}<b_{c}^{+}\left(x^{\prime}\right)\right\} .
\end{aligned}
$$

Therefore, the set of all the IPs, $I^{-}[\gamma] \neq V$, can be identified with $B^{+}(M)$. When $b_{c}^{+} \equiv \infty$ then $I^{-}[\gamma]=V$, and this TIP will be denoted $i^{+}$. Summing up:

Proposition 6.6 A subset $P \subset V$ (resp. $P \subsetneq V$ ) is an IP if and only if $P=$ $P\left(b_{c}^{+}\right)$for some Busemann function $b_{c}^{+}$(resp. for some finite Busemann function $\left.b_{c}^{+} \in B^{+}(M)\right)$.

Obviously, such a $b_{c}^{+}$is univocally determined by $P$. Therefore, the study of the future c-boundary of $(V, g)$ is reduced to the study of Busemann functions for the Finsler manifold $\left(M, F^{+}\right)$. More precisely, choose any $x_{0}$ in $M$, and notice that the following map is bijective,

$$
\pi_{x_{0}}^{+}: B^{+}(M) \rightarrow \mathbb{R} \times M_{B}^{+} \quad b_{c}^{+} \mapsto\left(b_{c}^{+}\left(x_{0}\right),\left[b_{c}^{+}\right]\right)
$$

$\left(\pi_{x_{0}}^{+}\right.$may be non-continuous, see Section 6.5). Identifying each element of $B^{+}(M)$ with its image under $\pi_{x_{0}}^{+}$, we can establish the following identification at a point set 
level:

$$
\begin{aligned}
\hat{V} & =B^{+}(M) \cup\{\infty\}=\left(\mathbb{R} \times M_{B}^{+}\right) \cup\left\{i^{+}\right\}=\left(\mathbb{R} \times\left(M \cup \partial_{B}^{+} M\right)\right) \cup\left\{i^{+}\right\} \\
& =\left(\mathbb{R} \times\left(M \cup \partial_{C}^{+} M \cup \partial_{\mathcal{B}}^{+} M\right)\right) \cup\left\{i^{+}\right\} \\
& =V \cup\left(\mathbb{R} \times \partial_{C}^{+} M\right) \cup\left(\mathbb{R} \times \partial_{\mathcal{B}}^{+} M\right) \cup\left\{i^{+}\right\}, \\
\hat{\partial} V & =\left(\mathbb{R} \times \partial_{B}^{+} M\right) \cup\left\{i^{+}\right\}=\left(\mathbb{R} \times \partial_{C}^{+} M\right) \cup\left(\mathbb{R} \times \partial_{\mathcal{B}}^{+} M\right) \cup\left\{i^{+}\right\} .
\end{aligned}
$$

This suggests a structure of cone for $\hat{\partial} V$ described below (Defn. 6.9.

We proceed analogously for the past c-boundary. Consider a past-directed timelike curve $\gamma:[\alpha,-\Omega) \rightarrow V$ parametrized as $\gamma(t)=(-t, c(t))$. Then:

$$
\begin{aligned}
I^{+}[\gamma] & =\left\{\left(t^{\prime}, x^{\prime}\right) \in V: \gamma(t) \ll\left(t^{\prime}, x^{\prime}\right) \text { for some } t \in[\alpha,-\Omega)\right\} \\
& =\left\{\left(t^{\prime}, x^{\prime}\right) \in V:-t<t^{\prime}-d^{+}\left(c(t), x^{\prime}\right) \text { for some } t \in[\alpha,-\Omega)\right\} \\
& =\left\{\left(t^{\prime}, x^{\prime}\right) \in V: t^{\prime}>\lim _{t \rightarrow-\Omega}\left(-t+d^{-}\left(x^{\prime}, c(t)\right)\right)\right\} \\
& =\left\{\left(t^{\prime}, x^{\prime}\right) \in V: t^{\prime}>b_{c}^{-}\left(x^{\prime}\right)\right\} .
\end{aligned}
$$

Proposition 6.7 A subset $F \subset V$ (resp. $F \subsetneq V$ ) is an IF if and only if $F=$ $F\left(b_{c}^{-}\right)$for some Busemann function $b_{c}^{-}$(resp. for some finite Busemann function $\left.b_{c}^{-} \in B^{-}(M)\right)$.

Defining a bijection $\pi_{x_{0}}^{-}$analogous to $\pi_{x_{0}}^{+}$above, we also obtain the point set identifications:

$$
\begin{aligned}
\check{V} & =V \cup\left(\mathbb{R} \times \partial_{C}^{-} M\right) \cup\left(\mathbb{R} \times \partial_{\mathcal{B}}^{-} M\right) \cup\left\{i^{-}\right\} \\
\check{\partial} V & =\left(\mathbb{R} \times \partial_{C}^{-} M\right) \cup\left(\mathbb{R} \times \partial_{\mathcal{B}}^{-} M\right) \cup\left\{i^{-}\right\} .
\end{aligned}
$$

Convention 6.8 (A) When a point $x_{0} \in M$ has been chosen as above, any set $P \in \hat{\partial} V \backslash\left\{i^{+}\right\}$in the future causal boundary will be determined univocally by a pair $\left(\Omega, x^{+}\right) \in \mathbb{R} \times \partial_{B}^{+} M$, according to 6.4). Analogously, any terminal set $F \in \check{\partial} V \backslash\left\{i^{-}\right\}$ in the past causal boundary will be represented by a pair $\left(\Omega, x^{-}\right) \in \mathbb{R} \times \partial_{B}^{-} M$.

(B) According to (5.6) and the conventions in Subsection 5.2.5 any non-proper forward or backward Busemann function $b_{c}^{ \pm}$determines univocally a pair $p=$ $\left(\Omega, x^{ \pm}\right) \in \mathbb{R} \times \partial_{C}^{ \pm} M$ such that

$$
b_{c}^{ \pm}(\cdot)=d_{p}^{ \pm}(\cdot):=\Omega \mp d^{ \pm}\left(\cdot, x^{ \pm}\right)=\Omega \mp d_{x^{ \pm}}^{ \pm}(\cdot) .
$$

(By Prop. 3.32, the domain of these functions can be extended to points of the form $\mathbb{R} \times\left(\partial_{C}^{+} M \cup \partial_{C}^{-} M\right)$.) Therefore, we can write

$$
B^{ \pm}(M) \backslash \mathcal{B}^{ \pm}(M) \equiv \mathbb{R} \times \partial_{C}^{ \pm} M
$$

independently of the choice of any point $x_{0}$ as above.

(C) In what follows, we will use the intrinsic decomposition (6.5) explained in (B), except when a point $x_{0}$ has been chosen explicitly, and the decomposition (6.4) is used. Thus, the pair $\left(\Omega, x^{ \pm}\right)$will be considered as explained in (A) (for all the 
boundary points but $i^{ \pm}$) or in (B) (for points associated to the Cauchy boundary) depending on the context. In each case, we will say that the TIP $P$ or the TIF $F$ is associated to the pair $\left(\Omega, x^{ \pm}\right)$. Moreover, as the projection 6.4 on the second factor is independent of the chosen $x_{0}$, we can also say that $\left(\Omega, x^{ \pm}\right)$is associated to $x^{ \pm}$, and that $\left(\pi^{ \pm}\right)_{x_{0}}^{-1}\left(x^{ \pm}\right)$is the line on $x^{ \pm}$.

In order to express previous results more compactly, we introduce the following definition:

Definition 6.9 For any topological space $T$, the forward cone with base $T$ is the quotient topological space $((-\infty, \infty] \times T) / \sim$ where the unique non-trivial identifications are $(\infty, x) \sim\left(\infty, x^{\prime}\right)$ for all $x, x^{\prime} \in T$. The class of $(\infty, x)$ will be called the apex of the cone.

For notational convenience, we will consider when necessary the backward cone, obtained by means of an analogous construction for $[-\infty, \infty) \times T$.

The results in this section have been obtained at a point set level, and can be summarized as follow:

Proposition 6.10 Let $(V, g)$ be a standard stationary spacetime. Fixing a point $x_{0} \in M$, the future (resp. past) c-completion $\hat{V}(\check{V})$ is identified naturally as a point set with a forward (resp. backward) cone with basis $M_{B}^{+}$(resp. $M_{B}^{-}$) and apex $i^{+}$(resp. $\left.i^{-}\right)$.

Moreover, the future (resp. past) c-boundary $\partial \hat{V}$ (resp. $\partial$ V) is formed by a cone with basis $\partial_{B}^{+} M=\partial_{C}^{+} M \cup \partial_{\mathcal{B}}^{+} M$ (resp. $\partial_{B}^{-} M=\partial_{C}^{-} M \cup \partial_{\mathcal{B}}^{-} M$ ) and apex $i^{+}$(resp. $\left.i^{-}\right)$.

The extension of this result to a topological level will be studied in Section 6.5.

\subsection{The (total) c-boundary as a point set}

Next, we are going to construct the (total) c-boundary $\partial V$ from the partial (future and past) ones. To this aim, it is essential to study which TIPs and TIFs are S-related, according to Section 2 (recall (2.1), (2.2)).

Consider a timelike curve $\gamma_{ \pm}:[\alpha, \pm \Omega) \rightarrow V$ parametrized as $\gamma_{ \pm}(t)=( \pm t, c(t))$ with $c \in C^{ \pm}(M)$. If $\pm \Omega=\infty$, the terminal set associated to $\gamma_{ \pm}$is S-related to the empty set, since the common future/past is empty (see Prop. 6.1). So, we will assume $\pm \Omega<\infty$. The following result determines the common future/past of $\gamma_{ \pm}$ in terms of $\Omega \in \mathbb{R}$ and the limit point of $c$ in $M_{C}^{ \pm}$.

Lemma 6.11 Consider a timelike curve $\gamma_{ \pm}:[\alpha, \pm \Omega) \rightarrow V$ parametrized as $\gamma_{ \pm}(t)=$ $( \pm t, c(t))$, with $\pm \Omega<\infty, c \in C^{ \pm}(M)$ and $c(t) \rightarrow x^{ \pm} \in M_{C}^{ \pm}$with the extended distance topology (Conv. 3.2\%) and denote $p=\left(\Omega, x^{ \pm}\right)$(Conv. 6.8 (B)). Then:

$$
\begin{gathered}
I^{-}\left[\gamma_{+}\right]=\left\{\left(t^{\prime}, x^{\prime}\right) \in V: t^{\prime}<d_{p}^{+}\left(x^{\prime}\right):=\Omega-d^{+}\left(x^{\prime}, x^{+}\right)\right\}=P\left(d_{p}^{+}\right) \\
\uparrow I^{-}\left[\gamma_{+}\right]=\left\{\left(t^{\prime}, x^{\prime}\right) \in V: t^{\prime}>d_{p}^{-}\left(x^{\prime}\right):=\Omega+d^{-}\left(x^{\prime}, x^{+}\right)\right\}=F\left(d_{p}^{-}\right) \\
\left(\begin{array}{l}
I^{+}\left[\gamma_{-}\right]=\left\{\left(t^{\prime}, x^{\prime}\right) \in V: t^{\prime}>d_{p}^{-}\left(x^{\prime}\right):=\Omega+d^{-}\left(x^{\prime}, x^{-}\right)\right\}=F\left(d_{p}^{-}\right) \\
\downarrow I^{+}\left[\gamma_{-}\right]=\left\{\left(t^{\prime}, x^{\prime}\right) \in V: t^{\prime}<d_{p}^{+}\left(x^{\prime}\right):=\Omega-d^{+}\left(x^{\prime}, x^{-}\right)\right\}=P\left(d_{p}^{+}\right)
\end{array}\right) .
\end{gathered}
$$


Proof. The expressions for $I^{-}\left[\gamma_{+}\right]$are straightforward from (6.3), and we focus on:

$$
\begin{aligned}
\uparrow I^{-}\left[\gamma_{+}\right] & =I^{+}\left[\left\{\left(t^{\prime}, x^{\prime}\right) \in V: \gamma_{+}(t) \ll\left(t^{\prime}, x^{\prime}\right) \text { for all } t\right\}\right] \\
& =I^{+}\left[\left\{\left(t^{\prime}, x^{\prime}\right) \in V: d^{-}\left(x^{\prime}, c(t)\right)<t^{\prime}-t \text { for all } t\right\}\right] \\
& =I^{+}\left[\left\{\left(t^{\prime}, x^{\prime}\right) \in V: t+d^{-}\left(x^{\prime}, c(t)\right)<t^{\prime} \text { for all } t\right\}\right] .
\end{aligned}
$$

Note that the function $t \mapsto t+d^{-}\left(x^{\prime}, c(t)\right)$ increases monotonically. In fact, since $c \in C^{+}(M)$, and so, $F^{+}(\dot{c})<1$, it is

$$
d^{-}\left(x^{\prime}, c\left(t_{1}\right)\right)-d^{-}\left(x^{\prime}, c\left(t_{2}\right)\right) \leq d^{+}\left(c\left(t_{1}\right), c\left(t_{2}\right)\right)<t_{2}-t_{1} \text { for } t_{1}<t_{2} .
$$

Therefore,

$$
\begin{aligned}
\uparrow I^{-}\left[\gamma_{+}\right] & =I^{+}\left[\left\{\left(t^{\prime}, x^{\prime}\right) \in V: \lim _{t} \nearrow_{\Omega}\left(t+d^{-}\left(x^{\prime}, c(t)\right)\right) \leq t^{\prime}\right\}\right] \\
& =\left\{\left(t^{\prime}, x^{\prime}\right) \in V: t^{\prime}>d_{p}^{-}\left(x^{\prime}\right)\right\},
\end{aligned}
$$

the last equality for $p=\left(\Omega, x^{+}\right)$, recalling that length $(c)<\infty, c(t) \rightarrow x^{+}$and Prop. 3.32. Finally, the expressions for $\gamma_{-}$are deduced similarly.

Remark 6.12 Observe that Propositions 6.6, 6.7 and Lemma6.11 remain true if we extend the set $C^{ \pm}(M)$ with curves of velocity lower or equal to one (recall Remark 4.17). Therefore, there is no difference if we consider timelike or causal curves for the computation of the c-completion i.e. the c-boundary of any standard stationary spacetime is properly causal (any inextensible future-directed causal curve will also have an endpoint), according to Defn. 3.33 and Th. 3.35 in [12].

With previous lemma at hand, the following result can be established.

Proposition 6.13 If $P \in \hat{\partial} V, F \in \check{\partial} V$ are represented by the same point $\left(\Omega, x^{s}\right) \in$ $\mathbb{R} \times \partial_{C}^{s} M$ then they are $S$-related, $P \sim_{S} F$. In particular, they define a unique element $(P, F) \in \partial V$.

Proof. Just observe that, from previous proposition,

$$
P=\downarrow F, \quad F=\uparrow P,
$$

which provides the desired result.

This proposition establishes identifications between those TIPs and TIFs associated to the same point of $\mathbb{R} \times \partial_{C}^{s} M$. Examples 6.19 and 6.20 illustrate situations where additional identifications between terminal sets appear; concretely, between TIPs and TIFs associated to points in $\partial_{C}^{+} M \backslash \partial_{C}^{s} M$ and $\partial_{C}^{-} M \backslash \partial_{C}^{s} M$, respectively. Remarkably, these identifications cannot appear in static spaces as $F^{+}=F^{-}$(see also [1]), and so, they are characteristic of the standard stationary case. In the following proposition we provide some restrictions for these additional identifications.

Proposition 6.14 Let $P, F$ be two terminal sets associated to $\left(\Omega^{+}, x^{+}\right),\left(\Omega^{-}, x^{-}\right)$ resp., where $\Omega^{+}, \Omega^{-} \in \mathbb{R}$ and $x^{ \pm} \in \partial_{C}^{ \pm} M \backslash \partial_{C}^{s} M$. If $P \sim_{S} F$, then $\Omega^{-}-\Omega^{+}=$ $d^{-}\left(x^{-}, x^{+}\right)$. 
Proof. Assume by contradiction that $\Omega^{-}-\Omega^{+} \neq d^{-}\left(x^{-}, x^{+}\right)$and consider the cases:

(A) $\Omega^{-}-\Omega^{+}<d^{-}\left(x^{-}, x^{+}\right)$. Take $\Omega^{-}<t<\Omega^{+}+d^{-}\left(x^{-}, x^{+}\right)$and consider a sequence $\left\{\left(t, x_{n}\right)\right\}_{n} \subset V$ such that $x_{n} \rightarrow x^{-}$with the topology associated to the extended distance $d^{-}$. Then:

$$
\lim _{n}\left(\Omega^{-}+d^{-}\left(x_{n}, x^{-}\right)\right)=\Omega^{-}<t<\Omega^{+}+d^{-}\left(x^{-}, x^{+}\right)=\lim _{n}\left(\Omega^{+}+d^{-}\left(x_{n}, x^{+}\right)\right) .
$$

Therefore, if we take $n_{0} \in \mathbb{N}$ such that $\Omega^{-}+d^{-}\left(x_{n_{0}}, x^{-}\right)<t<\Omega^{+}+d^{-}\left(x_{n_{0}}, x^{+}\right)$, then $\left(t, x_{n_{0}}\right) \in F$ and $\left(t, x_{n_{0}}\right) \notin \uparrow P$, in contradiction with $P \sim_{S} F$.

(B) $\Omega^{-}-\Omega^{+}>d^{-}\left(x^{-}, x^{+}\right)$. Take $\bar{\Omega}=\Omega^{+}+d^{-}\left(x^{-}, x^{+}\right)$and denote by $\bar{F}$ the TIF associated to $\left(\bar{\Omega}, x^{-}\right)$. As $d_{\left(\Omega^{-}, x^{-}\right)}^{-}>d_{\left(\bar{\Omega}, x^{-}\right)}^{-}$, one has $F \varsubsetneqq \bar{F}$. Therefore:

$$
\begin{aligned}
(t, x) \in \bar{F} & \Rightarrow t>\bar{\Omega}+d^{-}\left(x, x^{-}\right) \\
& \Rightarrow t>\Omega^{+}+d^{-}\left(x^{-}, x^{+}\right)+d^{-}\left(x, x^{-}\right) \geq \Omega^{+}+d^{-}\left(x, x^{+}\right) \\
& \Rightarrow(t, x) \in \uparrow P .
\end{aligned}
$$

Hence $(F \varsubsetneqq) \bar{F} \subset \uparrow P$, in contradiction to the maximality of $F$ in $\uparrow P$ required from $P \sim_{S} F$.

Summing up:

Theorem 6.15 Let $V=\mathbb{R} \times M$ be a standard stationary spacetime. Then, those pairs $(P, F)$ in the causal boundary $\partial V$ which have $P \neq \emptyset$, satisfy $P=P\left(b_{c}^{+}\right)$for some $c \in C^{+}(M)$, and:

(a) If $b_{c}^{+} \equiv \infty$ then $P=V, F=\emptyset$.

(b) If $b_{c}^{+} \in \mathcal{B}^{+}(M)$ then $F=\emptyset$.

(c) If $b_{c}^{+} \in B^{+}(M) \backslash \mathcal{B}^{+}(M)\left(\equiv \mathbb{R} \times \partial_{C}^{+} M\right)$, then $b_{c}^{+}=d_{p}^{+}$with $p=\left(\Omega^{+}, x^{+}\right) \in$ $\mathbb{R} \times \partial_{C}^{+} M$ and $F \subseteq F\left(d_{p}^{-}\right)$. In this case, there are two exclusive possibilities:

(c1) either $F=\emptyset$,

(c2) or $F=F\left(d_{p^{\prime}}^{-}\right)$with $p^{\prime}=\left(\Omega^{-}, x^{-}\right) \in \mathbb{R} \times \partial_{C}^{-} M$ and satisfying $\Omega^{-}-\Omega^{+}=$ $d^{-}\left(x^{-}, x^{+}\right)$(in this case, $p^{\prime}$ is not necessarily unique).

Moreover, if $x^{+} \in \partial_{C}^{s} M$, then (c2) holds with $p^{\prime}=p, \uparrow P=F\left(d_{p}^{-}\right)$and $P$ is univocally $S$-related with $F=F\left(d_{p}^{-}\right)$.

$A$ dual result holds for pairs $(P, F) \in \partial V$ with $F \neq \emptyset$.

Observe that, for $P \neq \emptyset$, previous theorem determines a unique $F$ such that $P \sim_{S} F$ except in the particular case when $P=P\left(d_{p}^{+}\right)$and $p=\left(\Omega^{+}, x^{+}\right) \in$ $\mathbb{R} \times\left(\partial_{C}^{+} M \backslash \partial_{C}^{s} M\right)$. The presence of these exceptions is related with the boundedness of the quasi-distance $d_{Q}^{+}$associated to the generalized metric space $\left(M, d^{+}\right)$(recall Remark 3.33. In fact, these exceptions will not appear if we suppose that $d_{Q}^{+}$is a generalized distance: 
Corollary 6.16 If $\left(M, d^{+}\right)$is a generalized metric space with either forward or backward evenly pairing boundary, then the c-completion of $V$ is simple as a point set (according to Def. 2.4). In particular, this happens if $d_{Q}^{+}$is a generalized distance.

Proof. The proof follows by analyzing the case (c2) in Theorem 6.15. In fact, if $P=P\left(d_{p}^{+}\right)$with $p=\left(\Omega, x^{+}\right) \in \mathbb{R} \times\left(\partial_{C}^{+} M \backslash \partial_{C}^{s} M\right)$, then $\uparrow P=\emptyset$ (recall Lemma 6.11 and Definition 3.34. For the last assertion recall Corollary 3.35.

Finally, recall that each point $x^{ \pm}=\left[b_{c}^{ \pm}\right] \in \partial_{B}^{ \pm} M$ determines in a natural way a line $\left\{b_{c}^{ \pm}+k: k \in \mathbb{R}\right\}$ in $\hat{\partial} V, \check{\partial} V$ (Convention $6.8(\mathrm{C})$ ) and then a (point set) cone. If $V$ has a c-completion which is simple as a point set, such lines also yield a simple picture of the c-boundary: $\partial V$ is composed by all the lines in $\hat{\partial} V$ and $\check{\partial} V$. But, when the point in $\partial_{B}^{ \pm} M$ belongs to $\partial_{C}^{s} M$, then the lines for $\hat{\partial} V$ and $\check{\partial} V$ must be identified as a single one - they represent $S$-paired elements. If the c-completion is not simple, the pairings are not so trivial. However, the structure of lines can be also defined, as we do now for future referencing.

Definition 6.17 Consider a point $(P, F) \in \partial V$ with $\emptyset \neq P=P\left(b_{c}^{+}\right)$. Then, we define the line over $(P, F)$, which will be denoted $L(P, F)$, as:

- If $F=\emptyset$ then $L(P, \emptyset):=\left\{\left(P^{\prime}, \emptyset\right): P^{\prime}=P\left(b_{c}^{+}+k\right), k \in \mathbb{R}\right\}$.

- If $F \neq \emptyset$, and so $P=P\left(d_{p}^{+}\right), F=F\left(d_{p^{\prime}}^{-}\right)$(recall Theorem 6.15), then

$$
L(P, F):=\left\{\left(P^{\prime}, F^{\prime}\right): P^{\prime}=P\left(d_{p}^{+}+k\right), F^{\prime}=F\left(d_{p^{\prime}}^{-}+k\right), k \in \mathbb{R}\right\} .
$$

$A$ dual definition is assumed for $(P, F) \in \partial V$ with $\emptyset \neq F=F\left(b_{c}^{-}\right)$.

Remark 6.18 Of course, if $P \neq \emptyset \neq F$, then the two definitions of line agree. Moreover, from the definition of the $S$-relation, if $P \sim_{S} F$ with $P=P\left(d_{p}^{+}\right), F=$ $F\left(d_{p^{\prime}}^{-}\right)$, then necessarily $P^{\prime} \sim_{S} F^{\prime}$ with $P^{\prime}=P\left(d_{p}^{+}+k\right), F^{\prime}=F\left(d_{p^{\prime}}^{-}+k\right)$ and $k \in \mathbb{R}$. So, the definition of line for $P \neq \emptyset$ is consistent.

Finally, we provide a pair of examples which illustrate the non-obvious possibilities in Theorem 6.15.

Example 6.19 (The possibility $p^{\prime} \neq p$ in case (c2) of Theorem 6.15 can hold.) Consider a standard stationary spacetime as explained in Figure 2, Recall that this example satisfies

$$
\partial_{B}^{+} M=\partial_{C}^{+} M=\left\{z^{+}\right\}, \quad \partial_{B}^{-} M=\partial_{C}^{-} M=\left\{z^{-}\right\} \quad \text { and } \quad 0<d^{+}\left(z^{+}, z^{-}\right)<\infty .
$$

These properties imply that, if $P$ is a TIP associated to $z^{+}$, then $\uparrow P$ is non-empty (recall Lemma 6.11), and one can find $S$-related non-empty sets as follows. Take a maximal IF, $F$, into $\uparrow P$. Notice that $F$ must be terminal: otherwise, any chain generating $P$, and so converging to $F=I^{+}(p)$ with the past chronological topology (recall that $F$ is maximal in $\uparrow P$ ), must also converge to $p$ with the topology of the manifold (since both topologies coincide on $V$ ), in contradiction to the terminal character of $P$. Hence, the TIF $F(\neq \emptyset)$ is associated to $p^{-}=\left(\Omega^{-}, z^{-}\right)$for some $\Omega^{-}$. As $P \subset \downarrow F$, let $P^{\prime} \supset P$ be some maximal IP into $\downarrow F$. Analogously, $P^{\prime}$ is associated to $p^{+}=\left(\Omega^{+}, z^{+}\right)$for some $\Omega^{+}$. By construction, $P^{\prime} \sim_{S} F$ with $p^{+} \neq p^{-}$. 


$$
\begin{aligned}
\partial_{C}^{+} M & \equiv\left\{z_{1}^{+}, z_{2}^{+}\right\} \\
\partial_{C}^{-} M & \equiv\left\{z_{1}^{-}, z_{2}^{-}\right\} \\
\partial_{C}^{s} M & =\emptyset
\end{aligned}
$$

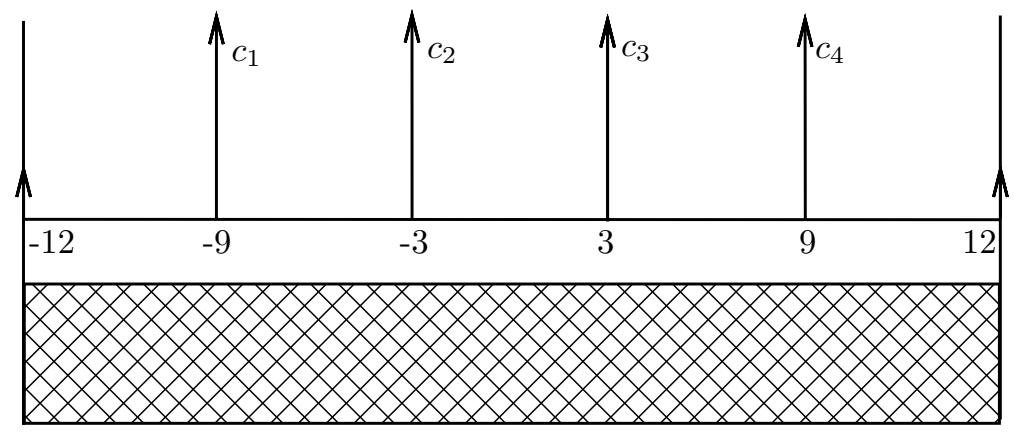

Figure 7: This example is constructed by sticking two spaces as in Figure 2, In particular, $c_{1}, c_{3}$ (resp. $c_{2}, c_{4}$ ) determine two points $z_{1}^{+}, z_{2}^{+} \in \partial_{C}^{+} M$ (resp. $z_{1}^{-}, z_{2}^{-} \in$ $\left.\partial_{C}^{-} M\right)$. Moreover, the distances $d\left(z_{i}^{+}, z_{j}^{-}\right)$are finite for $i, j=1,2$, but $z_{i}^{+}, z_{j}^{-} \notin \partial_{C}^{s} M$ (in fact, $\partial_{C}^{s} M=\emptyset$ ).

Example 6.20 (The suggested non-uniqueness of $p^{\prime}$ in Theorem 6.15 (c2) can hold.) Consider a standard stationary spacetime resulting from sticking two spaces as in previous example (see Figure 7). This spacetime is defined in such a way the following properties hold:

$$
\partial_{C}^{+}(M) \equiv\left\{z_{1}^{+}, z_{2}^{+}\right\}, \partial_{C}^{-}(M) \equiv\left\{z_{1}^{-}, z_{2}^{-}\right\}, d^{+}\left(z_{1}^{+}, z_{1}^{-}\right)=d^{+}\left(z_{2}^{+}, z_{2}^{-}\right)=r
$$

with $0<r<\infty$ and then $P \sim_{S} F, P^{\prime} \sim_{S} F, P \sim_{S} F^{\prime}, P^{\prime} \sim_{S} F^{\prime}$, where

$$
\begin{array}{ll}
P=\left\{(t, x): t<d_{\left(\Omega, z_{1}^{+}\right)}^{+}(x)\right\} & F=\left\{(t, x): t>d_{\left(\Omega+r, z_{1}^{-}\right)}^{-}(x)\right\} \\
P^{\prime}=\left\{(t, x): t<d_{\left(\Omega, z_{2}^{+}\right)}^{+}(x)\right\} & F^{\prime}=\left\{(t, x): t>d_{\left(\Omega+r, z_{2}^{-}\right)}^{-}(x)\right\} .
\end{array}
$$

So, we have the existence of four terminal sets, $P, P^{\prime}, F, F^{\prime}$ satisfying

$$
(P, F),\left(P, F^{\prime}\right),\left(P^{\prime}, F\right),\left(P^{\prime}, F^{\prime}\right) \in \partial V .
$$

Remark 6.21 The examples above become also relevant for the following discussions on the foundations of the notion of "causal boundary":

(a) The idea that some TIP may be S-paired with more than one TIF was stressed by Marolf and Ross [26, Figure 3]. However, their example might be regarded as somewhat "artificial' (namely, some topological manipulations are carried out in their construction). Example 6.20 yields the same effect without topological manipulations.

(b) By modifying Marolf and Ross' example, Flores constructed a new one which stressed that the set of all the S-related pairs could yield a "non-minimal" completion. Again, this effect is reproduced by the "less artificial" Example 6.20. The 
choice of non-minimal completions in the definition of c-boundary is discussed in [12, 30.

(c) Even when the pairs $(P, F)$ with $P \neq \emptyset \neq F$ are univocally determined by $P$ (or $F$ ), the highly non-trivial identification in Example 6.19 stresses the limitations of the conformal boundary [12, Figure 11, 12]. Such non-trivial identifications have been relevant to understand the AdS-CFT correspondence in plane waves (see 25, 13] and references therein).

\subsection{Causality of the c-boundary}

In this section we are going to study the causal relations between points of the cboundary of a standard stationary spacetime. Recall that in Section 2.1 we defined the chronological relation between points of the c-completion, but not a causal relation which extends the relation $\leq$ on the spacetime to pairs $(P, F),\left(P^{\prime}, F^{\prime}\right) \in \bar{V}$. For our purposes, it is enough to consider the following obvious sufficient criterium for $(P, F),\left(P^{\prime}, F^{\prime}\right) \in \bar{V}$ with either $P \neq \emptyset$ or $F^{\prime} \neq \emptyset$ :

$$
P \subset P^{\prime} \text { and } F^{\prime} \subset F \Rightarrow(P, F) \leq\left(P^{\prime}, F^{\prime}\right) .
$$

In the particular case of standard stationary spacetimes (which have a properly causal c-boundary, see Remark 6.12), this criterium can be also regarded as the definition of $\leq$, but we will not discuss this point here. Simply, we point out that a general definition of $\leq$ in $\bar{V}$ should take into account that the chronological relation always induces a causal relation (see [27, Defn. 2.22, Th. 3.69]); we refer to [12, Sect. 3.5] and [26, Sect. 3.2] for further discussions.

We focus on causal relations between points of the same line (Definition 6.17). To this aim, we introduce the following definitions, which interpret the causal relations in our case:

Definition 6.22 Consider a pair $(P, F)$ with $P=P\left(b_{c}^{+}\right)$and $F=F\left(b_{c^{\prime}}^{-}\right)$(or $F=\emptyset)$, and consider two arbitrary pairs

$$
\left(P\left(b_{c}^{+}+k_{1}\right), F\left(b_{c^{\prime}}^{-}+k_{1}\right)\right),\left(P\left(b_{c}^{+}+k_{2}\right), F\left(b_{c^{\prime}}^{-}+k_{2}\right)\right) \in L(P, F) .
$$

Then, the line $L(P, F)$ is:

- Timelike if for $k_{1}<k_{2},\left(P\left(b_{c}^{+}+k_{1}\right), F\left(b_{c^{\prime}}^{-}+k_{1}\right)\right) \ll\left(P\left(b_{c}^{+}+k_{2}\right), F\left(b_{c^{\prime}}^{-}+k_{2}\right)\right)$.

- Horismotic if for $k_{1}<k_{2},\left(P\left(b_{c}^{+}+k_{1}\right), F\left(b_{c^{\prime}}^{-}+k_{1}\right)\right) \leq\left(P\left(b_{c}^{+}+k_{2}\right), F\left(b_{c^{\prime}}^{-}+k_{2}\right)\right)$, but they are not chronologically related.

- Locally horismotic if, for each $k_{1}$, there exists $\delta>0$ such that for any $k_{2} \in \mathbb{R}$ with $\left|k_{2}-k_{1}\right|<\delta,\left(P\left(b_{c}^{+}+k_{1}\right), F\left(b_{c^{\prime}}^{-}+k_{1}\right)\right)$ and $\left(P\left(b_{c}^{+}+k_{2}\right), F\left(b_{c^{\prime}}^{-}+k_{2}\right)\right)$ are causally but not chronologically related.

Note that, for a curve in a spacetime, previous definitions of the causality of a line correspond with being timelike, lightlike with no cut points and lightlike, resp. 
Moreover, from the stationary character of the metric, $\delta$ in the definition of locally horismotic can be chosen independently of $k_{1}$.

The causal relations for the c-boundary of a standard stationary spacetime are summarized in the following proposition:

Proposition 6.23 Consider a point $(P, F) \in \partial V$ with $P \neq \emptyset$ and its associated line $L(P, F)$. Then:

(i) If $F=\emptyset$, then $L(P, \emptyset)$ is horismotic.

(ii) If $F \neq \emptyset$, and so, $P=P\left(d_{p}^{+}\right), F=F\left(d_{p^{\prime}}^{-}\right)$(necessarily, with $p=\left(\Omega, x^{+}\right) \in$ $\left.\mathbb{R} \times \partial_{C}^{+} M, p^{\prime}=\left(\Omega+d^{+}\left(x^{+}, x^{-}\right), x^{-}\right) \in \mathbb{R} \times \partial_{C}^{-} M\right)$. Then:

1. If $p=p^{\prime}, L(P, F)$ is timelike.

2. If $p \neq p^{\prime}, L(P, F)$ is locally horismotic.

Proof. (i) Consider two points in the line, $\left(P\left(b_{c}^{+}+k_{1}\right), \emptyset\right),\left(P\left(b_{c}^{+}+k_{2}\right), \emptyset\right) \in L(P, F)$. If $k_{1}<k_{2}$ then $P\left(b_{c}^{+}+k_{1}\right) \subset P\left(b_{c}^{+}+k_{2}\right)$ and $\emptyset \cap P\left(b_{c}^{+}+k_{2}\right)=\emptyset$. So, $\left(P\left(b_{c}^{+}+k_{1}\right), \emptyset\right)$ is causally related with $\left(P\left(b_{c}^{+}+k_{2}\right), \emptyset\right)$, but it is not chronologically related, i.e. the line is horismotic.

(ii) If $F \neq \emptyset$, and so, $P=P\left(d_{p}^{+}\right), F=F\left(d_{p^{\prime}}^{-}\right)$, reasoning as in previous paragraph we can check that $P\left(d_{p}^{+}+k_{1}\right) \subset P\left(d_{p}^{+}+k_{2}\right)$ and $F\left(d_{p^{\prime}}^{-}+k_{2}\right) \subset F\left(d_{p^{\prime}}^{-}+k_{1}\right)$ for $k_{1}<k_{2}$. Then, $\left(P\left(d_{p}^{+}+k_{1}\right), F\left(d_{p^{\prime}}^{-}+k_{1}\right)\right) \leq\left(P\left(d_{p}^{+}+k_{2}\right), F\left(d_{p^{\prime}}^{-}+k_{2}\right)\right)$. Now:

1. For $p=p^{\prime}=\left(\Omega, x^{s}\right) \in \mathbb{R} \times \partial_{C}^{s} M$, observe that $(\bar{\Omega}, \bar{x}) \in P\left(d_{p}^{+}+k_{2}\right) \cap F\left(d_{p}^{-}+k_{1}\right)$, with $\bar{\Omega}=\Omega+\left(k_{1}+k_{2}\right) / 2$ and $\bar{x} \in M$ such that $d^{ \pm}\left(\bar{x}, x^{s}\right)<\left(k_{2}-k_{1}\right) / 2$ (recall that $\left.x^{s} \in \partial_{C}^{s} M\right)$. In conclusion, both pairs are also chronologically related.

2. For $p \neq p^{\prime}$, the line $L(P, F)$ is locally horismotic. In fact, if two points of the line were chronologically related, then we would have $(t, x) \in P\left(d_{p}^{+}+k_{2}\right) \cap$ $F\left(d_{p^{\prime}}^{-}+k_{1}\right)$, for some $k_{2}>k_{1}$. Therefore, $t<\Omega+k_{2}$ and $t>\Omega+d^{+}\left(x^{+}, x^{-}\right)+$ $k_{1}$, which is only possible if $k_{2}-k_{1}>d^{+}\left(x^{+}, x^{-}\right)$.

Remark 6.24 This last point 2 in the previous proof is sharper in the determination of the locally horismotic character of the line $L(P, F)$ for $p \neq p^{\prime}$. In fact, when the two values $k_{1}, k_{2}$ of the parameter of the line satisfies $\left|k_{1}-k_{2}\right| \leq d^{+}\left(x^{+}, x^{-}\right)$, the corresponding points in $L(P, F)$ are not chronologically related. So, $d^{+}\left(x^{+}, x^{-}\right)$ behaves as a lower bound for the appearance of a "cut point" along $L(P, F)$.

\subsection{Topology of the partial boundaries and the c-boundary}

In Proposition 6.10, we established that the point set structures of the future completion and the future boundary can be regarded in a natural way as a cone. In fact, for any $x_{0}$ in $M$, the map $\pi_{x_{0}}^{+}$defined in $(6.4)$ is bijective.

In order to extend the cone structure of $\hat{V}$ and $\hat{\partial} V$ to a topological level, some issues on the chronological topology appear. On one hand, if the chronological 
topology differs from the pointwise one, the map $\pi_{x_{0}}^{+}$may be non-continuous (this happens when a sequence $\left\{f_{n}\right\}$ which converges chronologically to a function $f$, does not converge pointwise at $x_{0}$, i.e. $\left.\left\{f_{n}\left(x_{0}\right)\right\} \not \rightarrow f\left(x_{0}\right)\right)$. On the other hand, the continuity of $\pi_{x_{0}}^{+}$at some point may depend on the choice of $x_{0} \in M$ : observe that, in Example 4.9, the chronological and pointwise convergences of $\left\{x_{n}\right\}$ differ, but $\pi_{x_{0}}, x_{0}=(a, b)$, preserves the limit if, and only if, $b=1 / 2$ (see Figure 4 (B) for an intuitive idea).

At any case, the continuity will be ensured if we consider the pointwise topology. So, we are interested in the cases when both topologies, the chronological and the pointwise one, coincide. With this aim, note first (compare with Theorem 5.39):

Lemma 6.25 If $M_{B}^{+}$is Hausdorff then the pointwise and the chronological topologies coincide on $B^{+}(M)$.

Proof. Assume that $\left\{b_{c_{n}}^{+}\right\} \subset B^{+}(M)$ converges to some $b_{c}^{+} \in B^{+}(M)$ with the chronological topology, and let us prove that it also converges with the pointwise one (the converse follows from Prop. 5.29. From Lemma 5.38, $B^{+}(M)$ is ULL, thus, $\hat{L}$ is of first order (Proposition 5.37) and $b_{c}^{+} \in \hat{L}\left(\left\{b_{c_{n}}^{+}\right\}\right)$. Obviously, $\left\{\left[b_{c_{n}}^{+}\right]\right\} \subset$ $M_{B}^{+}$converges to $\left[b_{c}^{+}\right] \in M_{B}^{+}$with its own chronological topology, and so, it also converges with the pointwise one (as $M_{B}^{+}$is Hausdorff, Theorem 5.39 applies). Therefore, there exist some constants $\left\{k_{n}\right\} \subset \mathbb{R}$ such that $\left\{b_{c_{n}}^{+}-k_{n}\right\} \subset B^{+}(M)$ converges pointwise to $b_{c}^{+} \in B^{+}(M)$. Moreover, from the definition of chronological limit (see (5.7) ), $k_{n} \rightarrow 0$. In fact, otherwise, there are two possibilities: either $k_{n_{j}}>\epsilon>0$, or $k_{n_{j}}<-\epsilon<0$, for some subsequence $\left\{n_{j}\right\}_{j} \subset\{n\}$. In the first case $b_{c}^{+}+\epsilon / 2<\liminf b_{c_{n_{j}}}^{+}$, and so, $\left\{b_{c_{n}}^{+}\right\}$does not converge chronologically to $b_{c}^{+}$, since $b_{c}^{+}+\epsilon / 2$ violates the maximal character of $b_{c}^{+}$(recall (b) in (5.7)). In the second case, $b_{c}^{+}>\lim \inf _{n} b_{c_{n_{j}}}^{+}$, which violates condition (a) in 5.7).

Theorem 6.26 Let $V=\mathbb{R} \times M$ be a standard stationary spacetime. If $M_{B}^{+}$is Hausdorff then the map $\pi_{x_{0}}^{+}$in (6.4) is an homeomorphism for all $x_{0} \in M$.

So, in this case, the future causal completion $\hat{V}=B^{+}(M) \cup\{\infty\}$ is a (topological) cone with basis $M_{B}^{+}$and apex $i^{+}$, and the future causal boundary $\hat{\partial} V=B^{+}(M) \backslash V$ is also a (topological) cone with basis $\partial_{B}^{+} M$ and apex $i^{+}$.

Proof. By previous lemma, the subset $B^{+}(M)$ of $\hat{V}$ is endowed with the pointwise convergence topology, and then, $\pi_{x_{0}}^{+}$is obviously continuous. For the continuity of the inverse, observe that if $\left\{\left[b_{c_{n}}^{+}\right]\right\}$converges pointwise to $\left[b_{c}^{+}\right]$and $\left\{b_{c_{n}}^{+}\left(x_{0}\right)\right\}$ converges to $b_{c}^{+}\left(x_{0}\right)$, necessarily $\left\{b_{c_{n}}^{+}\right\}$converges pointwise to $b_{c}^{+}$. Finally, as $\left\{b_{c_{n}}^{+} \equiv\right.$ $\left.\left(t_{n}, x^{+}\right)\right\} \subset \mathbb{R} \times M_{B}^{+}$converges to $i^{+}$if and only if $t_{n} \rightarrow \infty$, we deduce that the future causal completion $\hat{V}$ (resp. the future causal boundary $\hat{\partial} V$ ) is a topological cone with base $M_{B}^{+}$(resp. $\left.\partial_{B}^{+} M\right)$ and apex $i^{+}$.

The definition of the chronological topology, combined with Theorem 6.15 and Proposition 6.23 , yields a full description of the c-completion of a stationary spacetime. Now, Theorem 6.26 shows that the topological structure of the partial boundaries is particularly simple when $M_{B}^{ \pm}$is Hausdorff. Even in this case, the topological 
structure of the (total) c-boundary presents some complications related with the ambiguity appeared at the point set level (recall Theorem 6.15). So, in order to ensure a well behaviour of the S-relation and obtain a nice description of the topology of the (total) c-boundary, additional conditions are required:

Theorem 6.27 Let $V=\mathbb{R} \times M$ be a standard stationary spacetime. If $M_{C}^{s}$ is locally compact and $d_{Q}^{+}$is a generalized distance, then $\bar{V}$ is simple, and so, $\bar{V}$ is homeomorphic to the quotient topological space $\left(\hat{V} \cup_{d} \check{V}\right) / \sim_{S}$.

Proof. According to Definition 2.4 and Proposition 2.5 (2), it suffices to show that a pair $(P, F) \in \bar{V}$ with $P \neq \emptyset \neq F$ is limit of a sequence $\left\{\left(P_{n}, F_{n}\right)\right\}$ whenever $P \in \hat{L}\left(P_{n}\right)$ or $F \in \check{L}\left(F_{n}\right)$. In fact, if say $P \in \hat{L}\left(P_{n}\right)$, let us see that $F \in \check{L}\left(F_{n}\right)$. Since $d_{Q}^{+}$is generalized and $P \neq \emptyset \neq F$, Theorem 6.15 (see also Corollary 6.16) implies that $P=P\left(d_{p}^{+}\right), F=F\left(d_{p}^{-}\right)$for some $p=\left(\Omega, x^{s}\right) \in \mathbb{R} \times M_{C}^{s}$. As $\overline{M_{C}^{s}}$ is locally compact, $P_{n}$ has to be of the form $P\left(d_{p_{n}}^{+}\right), p_{n}=\left(\Omega_{n}, x_{n}^{s}\right) \in \mathbb{R} \times M_{C}^{s}$, for $n$ big enough, and such that $x_{n}^{s} \rightarrow x^{s}$ (recall Prop. 5.24). Moreover, from Theorem 6.15 also $F_{n}=F\left(d_{p_{n}}^{-}\right)$. Since $p_{n} \rightarrow p$, it is $F \in L\left(F_{n}\right)$ (recall Lemma 5.5 and Proposition 5.29 .

Finally, summarizing previous results:

Corollary 6.28 Let $V=\mathbb{R} \times M$ be a standard stationary spacetime. If $d_{Q}^{+}$is a generalized distance, $M_{C}^{s}$ is locally compact and $M_{B}^{ \pm}$are Hausdorff, then $\bar{V}$ is simple

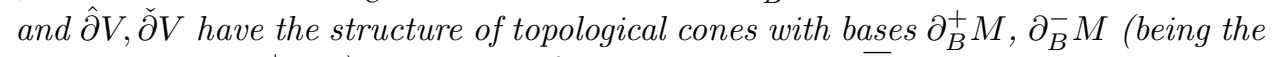
apexes denoted $i^{+}, i^{-}$) resp. Therefore, the completion $\bar{V}$ is homeomorphic to the quotient topological space $\hat{V} \cup_{d} \check{V} / \sim_{S}$, where $\hat{V}, \check{V}$ has the structure of topological cones with bases $M_{B}^{+}, M_{B}^{-}$(and same apexes $i^{+}, i^{-}$), resp.

Remark 6.29 Summarizing, Figure 9 represents the point set and causal structures of the c-completion of a standard stationary spacetime. The c-boundary illustrated in the figure is simple as a point set; if this condition did not hold, only the addition of new locally horismotic lines (as the depicted "helix") through pairs type $\left(P_{3}, F_{3}^{\prime}\right)$ or $\left(P_{3}^{\prime}, F_{3}\right)$ would be required. The first diagram in Figure 8 summarizes the implications at these pointset and chronological levels.

In order to ensure also the topological structure suggested by Figure 9, two conditions have to be imposed: the Hausdorffness of $M_{B}^{ \pm}$(to obtain the topological structure of double cone, recall Th. 6.26), and the (topological) simplicity of $\partial V$ (to ensure the good behavior of the identifications). The second diagram in Figure 8 summarizes when these properties are ensured. Recall that: (a) when $M_{B}^{ \pm}$is Hausdorff, both the chronological topology on $\bar{V}$ is also Hausdorff (recall Lemma 6.25 and the identifications between terminal sets and $B^{ \pm}(M)$ ), and $\bar{V}$ is simple as a point set (Proposition 2.5(1)), (b) the simplicity of $\partial V$ is ensured under the conditions in Theorem 6.27.

Particularized to the static case (when $d_{Q}^{+}$is directly a generalized distance), Theorem 6.26 culminates the study of $\hat{\partial} V$ in [20, 11], and Corollary 6.28 gives the correct topological description of $\partial V$, thus completing [1. Recall that the pairings 
for the boundary in the static case are trivial. So, Figure 10 gives a full intuitive picture of the point set and causal structures of the boundary, which is also valid at the topological level when $M_{C}$ is locally compact and $M_{B}$ Hausdorff.

\subsection{Proof of Theorem 1.2}

Now, we are in conditions to summarize the proof of Theorem 1.2 .

Proof of Theor. 1.2. Assertion (1A) of the point set structure follows from Proposition 6.10. Assertion (1B) follows from Theorem 6.15.

The assertion about the causal structure is summarized in Proposition 6.23.

Finally, about the topological structure, assertions (3A) and (3B) follow from Theorems 6.26 and 6.27 resp. The last paragraph is proved in Corollary 6.28.

\section{Acknowledgments}

The comments by one of the referees are acknowledged. The authors are partially supported by the Spanish grants MTM2010-18099 (MICINN) and P09-FQM-4496 (J. Andalucía), both with FEDER funds. Also, the second-named author is supported by the Ph.D. thesis Spanish MEC Grant AP2006-02237, 22. The thirdnamed author acknowledges IHES (Bures-sur-Yvette, France) a stay during the month of August 2010, about the topics of this paper.

\section{References}

[1] V. Alaña, J.L. Flores, The causal boundary of product spacetimes, Gen. Relat. Gravitation 39 (2007), no. 10, 1697-1718.

[2] W. Ballmann, M. Gromov, V. Schroeder, Manifolds of nonpositive curvature, Progress in Mathematics, 61. Birkhäuser Boston, Inc., Boston, MA, 1985.

[3] D. Bao, S.-S. Chern, Z. Shen, An introduction to Riemann-Finsler geometry, vol. 200 of Graduate Texts in Mathematics, Springer-Verlag, New York, 2000.

[4] J.K. Beem, P.E. Ehrlich, K.L. Easley, Global Lorentzian Geometry, Monographs Textbooks Pure Appl. Math. 202, Dekker Inc., New York (1996).

[5] R.M. Bridson, A. Haefliger, Metric spaces of non-positive curvature, Grundlehren der Mathematischen Wissenschaften, 319. Springer-Verlag, Berlin, 1999.

[6] A.M. Candela, J.L Flores, M. Sánchez, Global hyperbolicity and Palais-Smale condition for action functionals in stationary spacetimes, Adv. Math., 218 (2008) 515-536. 

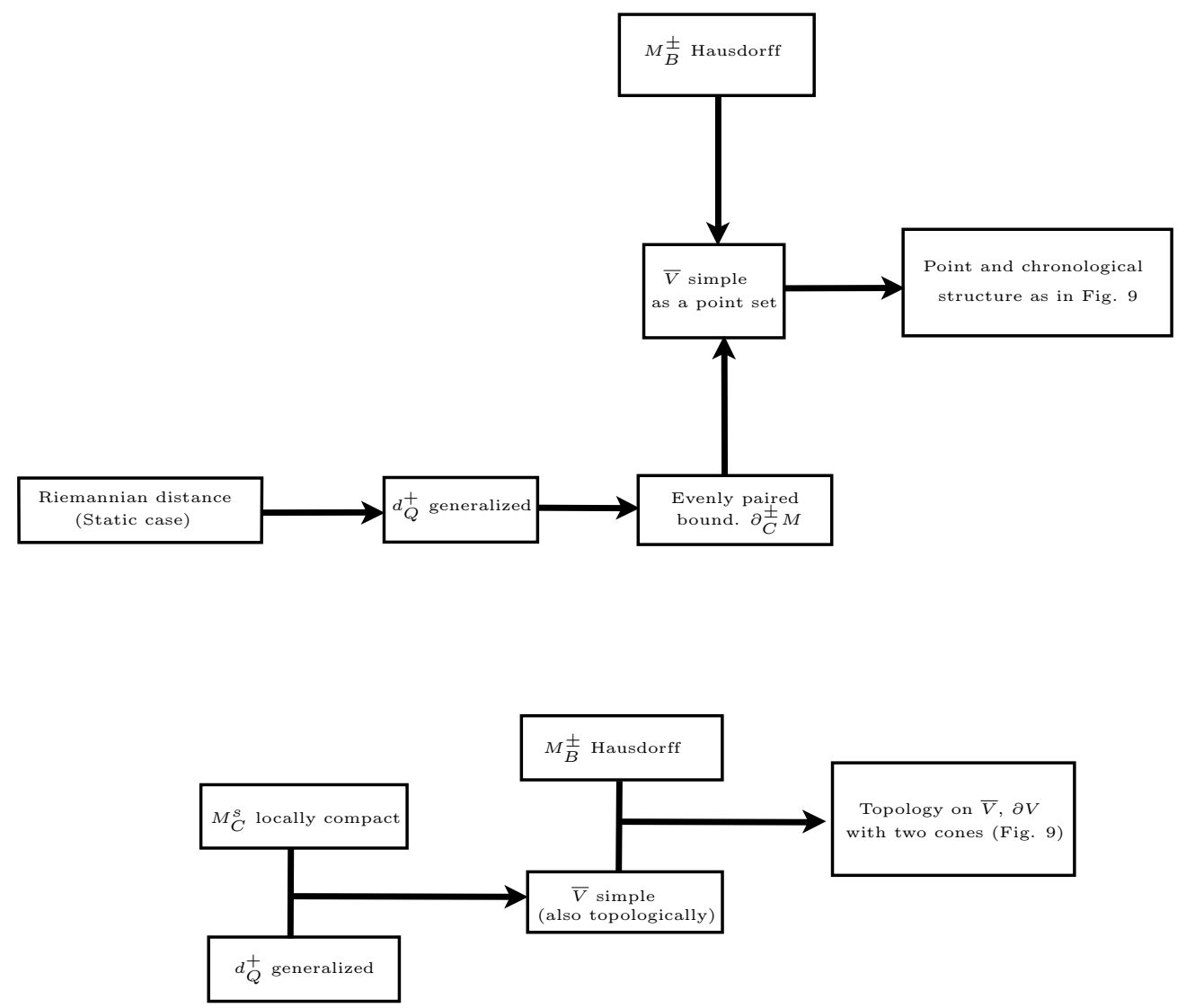

Figure 8: Sufficient general conditions which simplify the structure of the cboundary both, as a point set and topologically (the arrows mean "implies"), see Remark 6.29.

The character of simple as a point set for $\bar{V}$ (which is satisfied either if $M_{B}^{ \pm}$is Hausdorff or if $V$ is static), is enough to ensure that $\partial V$ includes a "natural double cone" as a point set. However, to ensure that this double cone appears also at the topological level, both conditions, $M_{B}^{ \pm}$is Hausdorff and $\bar{V}$ is simple (also topologically), are imposed. In terms of the geometry of $M$, the latter condition is ensured by imposing both, $M_{C}^{s}$ is locally compact and $d_{Q}^{+}$is a generalized distance. 


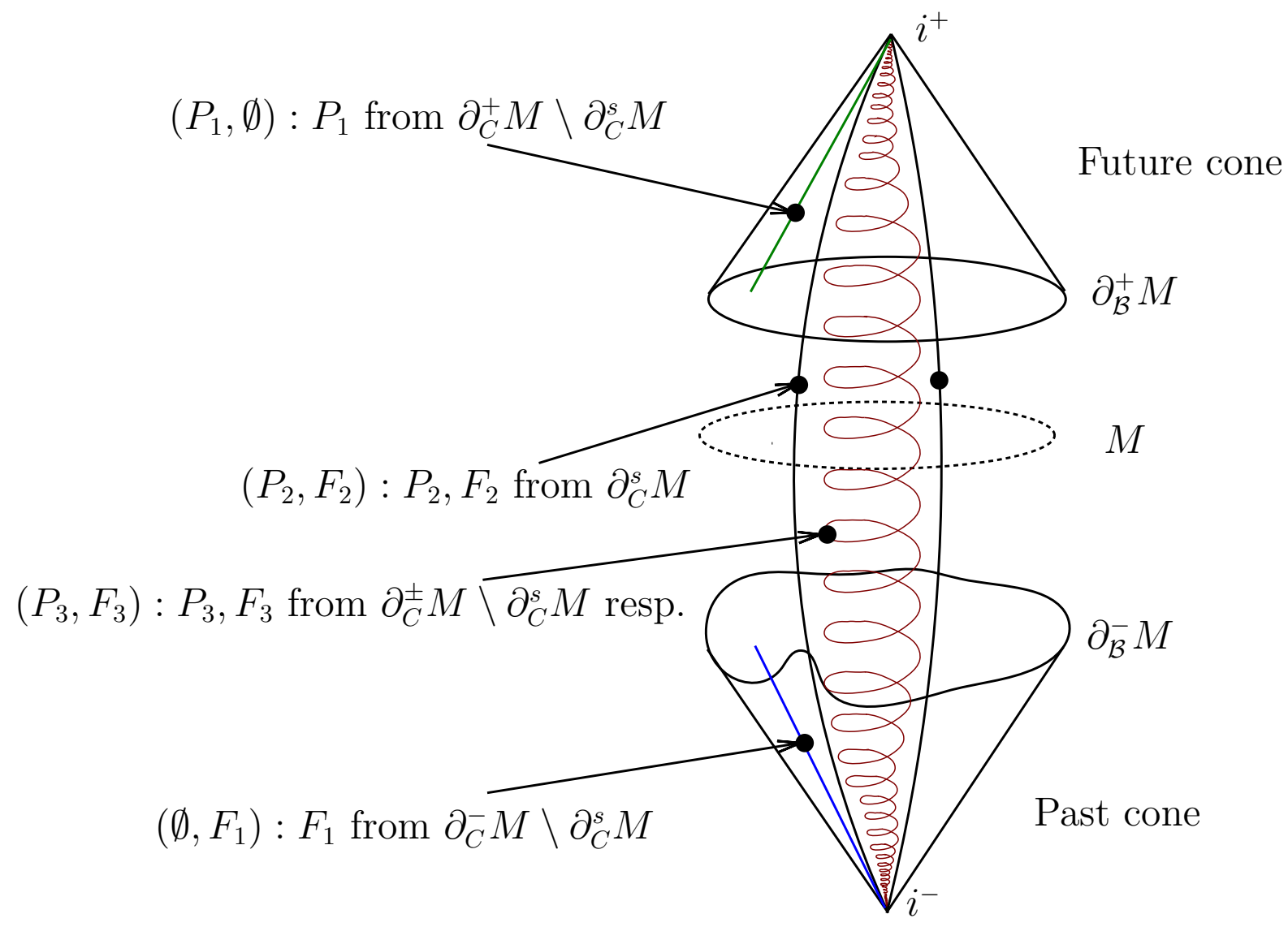

Figure 9: Possibilities for the c-boundary of a standard stationary spacetime:

The properly Busemann boundaries $\partial_{\mathcal{B}}^{ \pm} M$ determine the (forward, backward) directions of $M$ at infinity. Each one yields a cone in $\partial V$ with apex $i^{+}$or $i^{-}$and horismotic lines from the apex.

Each point $x^{+}$of the Cauchy boundary $\partial_{C}^{+} M$ determines one (or more) lines with different properties depending on if it generates $S$-relations (non-trivial pairings) with one (or more) points $x^{-} \in \partial_{C}^{-} M$ or not. If it does not, then $x^{+}$generates a single horismotic line [see $\left(P_{1}, \emptyset\right)$ in the figure; $\left(\emptyset, F_{1}\right)$ represents the dual situation for $\left.\partial_{C}^{-} M\right]$. In the case that $x^{+}$generates some $S$-relation, there are two possibilities: (i) $x^{+}$belongs to $\partial_{C}^{s} M$, and so, it generates a single line, which is timelike [see $\left.\left(P_{2}, F_{2}\right)\right]$; (ii) $x^{+}$belongs to $\partial_{C}^{+} M \backslash \partial_{C}^{s} M$ and generates one (or more) lightlike (i.e. locally horismotic) lines [represented as the "helix" through $\left.\left(P_{3}, F_{3}\right)\right]$. 


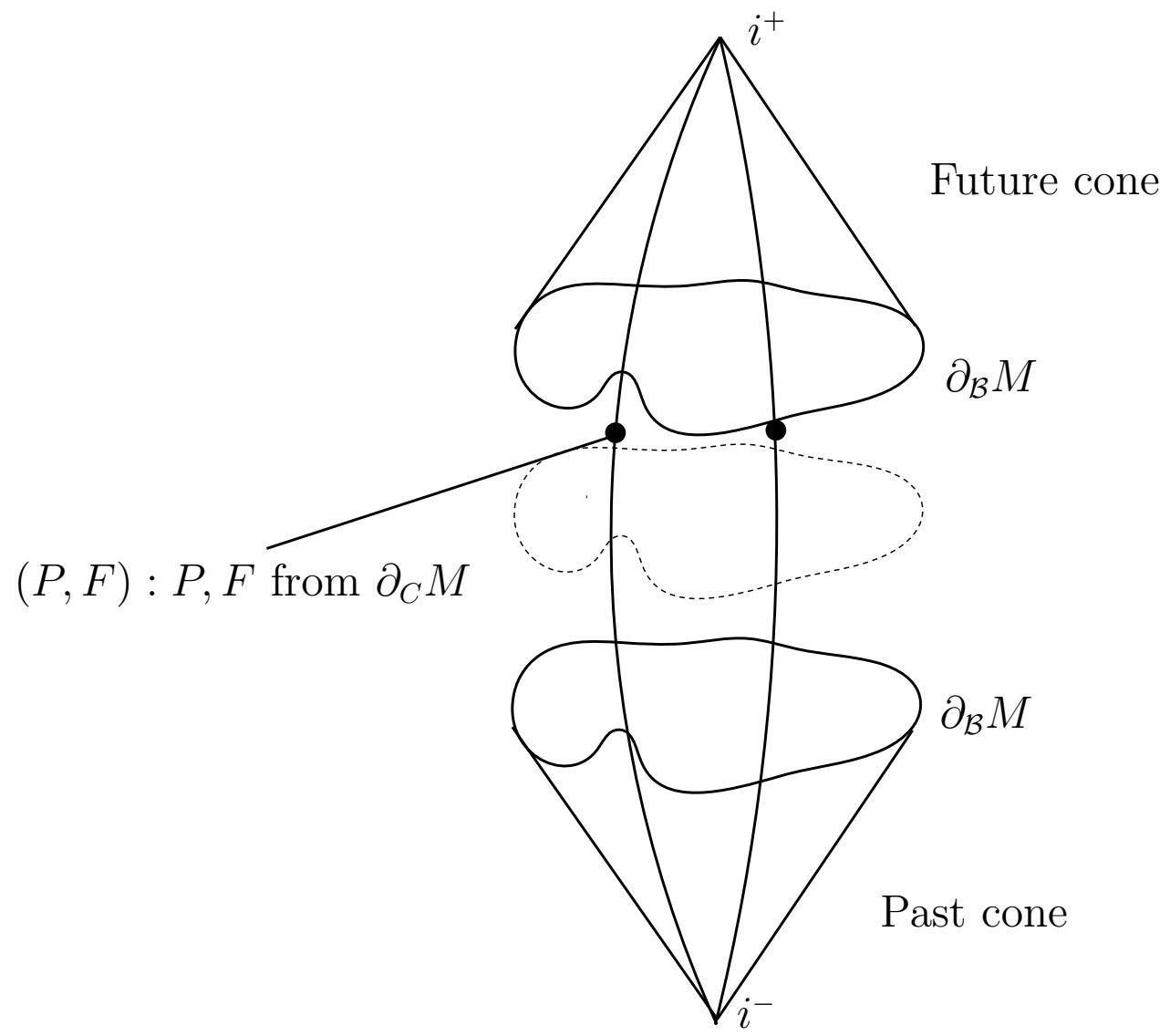

Figure 10: The static case (when the Finsler metric on $M$ becomes Riemmanian) satisfies that $\partial_{C}^{ \pm} M=\partial_{C}^{s} M=\partial_{C} M$ and $\partial_{\mathcal{B}}^{ \pm} M=\partial_{\mathcal{B}} M$; thus, the boundary is also symmetric under time reversal. As a point set, the c-boundary of a standard static spacetime is formed by two cones with bases $\partial_{\mathcal{B}} M$ and apexes $i^{+}, i^{-}$resp., and timelike lines associated to points of $\partial_{C} M$. However, to ensure this structure at the topological level, the local compactness of $M_{C}$ plus the Hausdorffness of $M_{B}$ must be imposed. 
[7] E. Caponio, M.A. Javaloyes, A. Masiello, On the energy functional on Finsler manifolds and applications to stationary spacetimes, Math. Ann. 351 (2011) 365-392.

[8] E. Caponio, M.A. Javaloyes, M. Sánchez, On the interplay between Lorentzian Causality and Finsler metrics of Randers type, Rev. Matem. Iberoamericana 27 (2011) 919-952.

[9] P. Eberlein, B. O'Neill, Visibility manifolds, Pacific J. Math. 46 (1973), 45109.

[10] J.L. Flores, The causal boundary of spacetimes revisited, Comm. Math. Phys. 276 (2007), 611-43.

[11] J.L. Flores, S.G. Harris, Topology of the causal boundary for standard static spacetimes, Class. Quant. Grav. 24 (2007), no. 5, 1211-1260.

[12] J.L. Flores, J. Herrera, M. Sánchez, On the final definition of the causal boundary and its relation with the conformal boundary, Adv. Theor. Math. Phys. 15 (2011), to appear. Available at arxiv: 1001.3270v3.

[13] J.L. Flores, M. Sánchez, The causal boundary of wave-type spacetimes, J. High Energy Phys. (2008), no. 3, 036, 43 pp.

[14] R.P. Geroch, E.H. Kronheimer, R. Penrose, Ideal points in spacetime, Proc. Roy. Soc. Lond. A 237 (1972) 545-567.

[15] G.W. Gibbons, C.A.R. Herdeiro, C.M. Warnick, and M.C. Werner, Stationary Metrics and Optical Zermelo-Randers-Finsler Geometry, Phys. Rev. D 79 (2009), no. 4, 044022, 21 pp.

[16] M. Gromov, Metric structures for Riemannian and non-Riemannian spaces, vol. 152 of Progress in Mathematics, Birkhäuser Boston Inc., Boston, MA, 1999 .

[17] M. Gromov, Hyperbolic manifolds, groups and actions. In: Riemann surfaces and related topics: Proceedings of the 1978 Stony Brook Conference (State Univ. New York, Stony Brook, N.Y., 1978), pp. 183-213. Ann. of Math. Stud., 97, Princeton Univ. Press, Princeton, N.J., 1981.

[18] S.G. Harris, Universality of the future chronological boundary, J. Math. Phys. 39 (1998), 5427.

[19] S.G. Harris, Topology of the future chronological boundary: universality for spacelike boundaries, Class. Quantum Grav. 17 (2000), 551-603.

[20] S.G. Harris, Causal boundary for standard static spacetimes, Nonlinear Anal. 47 (2001), 2971-81. 
[21] S.G. Harris, Boundaries on spacetimes: causality, topology, and group actions, Geom. Dedicata 126 (2007), 255-274.

[22] J. Herrera, El borde causal de un espaciotiempo y nuevos bordes en Geometría Diferencial, The causal boundary of a spacetime and new boundaries in Differential Geometry Ph. D. Thesis, U. Málaga, 2011.

[23] M.A. Javaloyes, M. Sánchez, Existence of standard splitting for conformally stationary spacetimes, Class. Quantum Grav. 25 (2008) 168001.

[24] J.L. Kelley, General topology, Van Nostrand, Princeton, N.J., 1967.

[25] D. Marolf, S. Ross, Plane Waves: To infinity and beyond!, Class. Quant. Grav. 19 (2002), 6289-302.

[26] D. Marolf, S.R. Ross, A new recipe for causal completions, Class. Quant. Grav. 20 (2003) 4085-4117.

[27] E. Minguzzi, M. Sánchez, The causal hierarchy of spacetimes, in Recent Developments in Pseudo-Riemannian Geometry, ESI Lect. Math. Phys., (Eur. Math. Soc. Publ. House, Zurich, 2008) p. 299-358, arXiv:gr-qc/0609119.

[28] J. Munkres, Topology, Prentice-Hall, 2nd edition.

[29] B. O'Neill, Semi-Riemannian Geometry with Applications to Relativity, Academic Press Inc. (1983).

[30] M. Sánchez, Causal boundaries and holography on wave type spacetimes, Nonlinear Anal. 71 (2009), no. 12, e1744-64.

[31] L.B. Szabados, Causal boundary for strongly causal spaces, Class. Quantum Grav. 5 (1988), 121-34.

[32] L.B. Szabados, Causal boundary for strongly causal spacetimes: II, Class. Quantum Grav. 6 (1989), 77-91.

[33] E. van Douwen, An anti-Hausdorff Fréchet space in which convergent sequences have unique limits Topology Appl. 51 (1993), no. 2, 147-158.

[34] E. M. Zaustinsky, Spaces with non-symmetric distance, Memoirs of the American Mathematical Society, 34 (1959). 